\title{
Excited-State Aromaticity Improves Molecular Motors: A Computational Analysis
}

Baswanth Oruganti, J un Wang and Bo Durbeej

The self-archived postprint version of this journal article is available at Linköping University Institutional Repository (DiVA):

http:// urn.kb.se/ resolve?urn=urn:nbn:se:liu:diva-141935

N.B.: When citing this work, cite the original publication.

Oruganti, B., Wang, J ., Durbeej, Bo, (2017), Excited-State Aromaticity Improves Molecular Motors: A Computational Analysis, Organic Letters, 19(18), 4818-4821.

https:// doi.org/ 10.1021/ acs.orglett.7b02257

Original publication available at:

https:/ / doi.org/ 10.1021/ acs.orglett.7b02257

Copyright: American Chemical Society

http:// pubs.acs.org/ 


\title{
Excited-State Aromaticity Improves Molecular Motors: A Computational Analysis
}

\author{
Baswanth Oruganti, Jun Wang* and Bo Durbeej* \\ Division of Theoretical Chemistry, IFM, Linköping University, SE-581 83 Linköping, Sweden \\ Supporting Information Placeholder
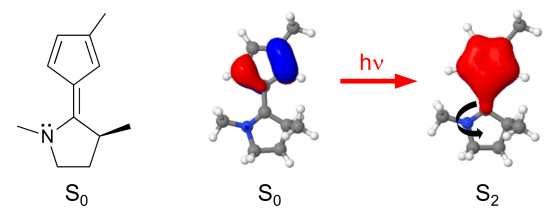 \\ $Z / E \rightarrow E / Z$ \\ rotary quantum yields $\approx 75 \%$ \\ excited-state lifetimes $\approx 200 \mathrm{fs}$
}

ABSTRACT: A new approach to the design of more efficient light-driven rotary molecular motors is presented and evaluated computationally based on molecular dynamics simulations. The approach involves enabling part of the motor to become aromatic in the photoactive excited state, and is found to sharply increase the rotary quantum yields of the photoisomerizations that underlie the motor function. Excited-state aromaticity thus holds promise as a guiding principle toward better-performing molecular motors.

Molecular motors are molecules that can perform net mechanical work using energy absorbed from an external source. Light-driven rotary molecular motors based on sterically overcrowded alkenes are the most developed class of synthetic molecular motors available today. ${ }^{1}$ Fueled by UV light and heat, these motors produce $360^{\circ}$ unidirectional rotary motion around a central olefinic bond connecting two molecular halves by means of consecutive photoisomerization and thermal isomerization steps. The rotary motion is controlled by the molecular chirality, which determines the preferred direction clockwise $(\mathrm{CW})$ or counterclockwise $(\mathrm{CCW})$ - of the photoisomerizations. ${ }^{\text {a }-\mathrm{d}}$

Although overcrowded-alkene motors have shown great potential for a wide variety of useful applications, ${ }^{2,3}$ their performance under ambient conditions is restrained in two different ways. First, the thermal isomerizations occur on much longer timescales than the photoisomerizations. ${ }^{1 \mathrm{c}, \mathrm{e}}$ Second, the photoisomerization quantum yields (QYs) are limited (to $\sim 20$ $30 \%$ ) by the unwanted pyramidalization of one of the central olefinic carbon atoms that accompanies the desired torsional motion. ${ }^{4,5}$ While much effort has been invested in accelerating the thermal steps of the motors ${ }^{1 b, c, e, 6}$ and in developing alternative light-driven motor designs that complete a full $360^{\circ}$ rotation without any thermal steps, ${ }^{7}$ successful attempts to address the second limitation and improve the photochemical efficiency are comparatively scarce. ${ }^{4,5,8}$

Recently, however, it has been shown that a motor design that incorporates a protonated or alkylated nitrogen Schiff base offers a potential solution to this challenge. ${ }^{5,8}$ Specifically, it has been found that the electron-withdrawing effect of the cationic nitrogen center on the isomerizing bond hinders the aforementioned pyramidalization, ${ }^{5}$ whereby the associated photoisomerizations can attain both higher QYs and shorter excited-state lifetimes than overcrowded-alkene motors. ${ }^{8}$
In this work, we present a new motor design that, despite lacking a cationic moiety, is able to produce fast unidirectional rotary motion with similar efficiency as Schiff-base motors by rather exploiting cyclic electron delocalization in an excited state (i.e., excited-state aromaticity). In particular, by performing both minimum energy path (MEP) calculations and nonadiabatic molecular dynamics (NAMD) simulations ${ }^{9}$ based on multiconfigurational quantum chemistry, ${ }^{10}$ we demonstrate that the concept of excited-state aromaticity ${ }^{11}$ holds substantial, yet hitherto unexplored, potential in the design of fast and efficient light-driven molecular motors. Indeed, although aromaticity is a well-established concept also for excited states, ${ }^{11}$ it has in the past mostly been used to rationalize the photochemical reactivity of triplet excited states. ${ }^{12}$

The motor design, hereafter referred to as motor $\mathbf{1}$ and shown in Scheme 1, features a cyclopentadiene motif connected by an olefinic bond to an electron-donating chiral $N$-methyl pyrrolidine framework. As we will see, key to the performance of $\mathbf{1}$ is that the cyclopentadiene motif, which is not aromatic in its ground state, nonetheless exhibits cyclic electron delocalization in the bright second excited singlet state $\left(\mathrm{S}_{2}\right)$ of $\mathbf{1}$.

Scheme 1. Photoinduced Cyclic Electron Delocalization in the $E$ Isomer of Motor 1 and Definitions of Dihedral Angles and Cyclopentadiene Bond Length Alternation (BLA)

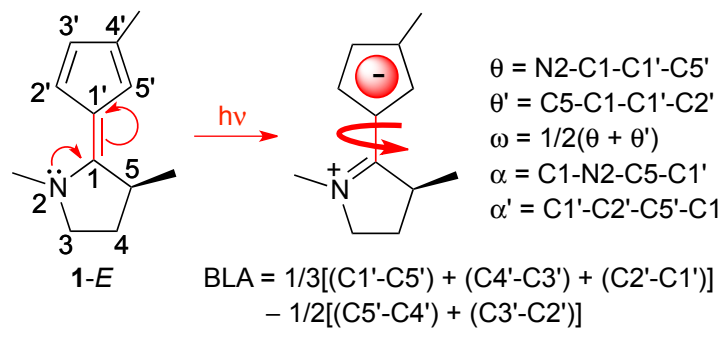


First, the ground-state $\left(\mathrm{S}_{0}\right)$ equilibrium geometries of the $E$ and $Z$ isomers of $\mathbf{1}$ with respect to the central olefinic bond were optimized with the complete active space self-consistent field (CASSCF) method. ${ }^{13}$ These and all other CASSCF-based calculations were performed with an active space of eight electrons ( $\operatorname{six} \pi$ and the nitrogen lone pair) in seven orbitals and, unless otherwise noted, the cc-pVTZ basis set. By subsequently calculating the two lowest excited singlet states $\left(\mathrm{S}_{1}\right.$ and $\mathrm{S}_{2}$ ) at the $\mathrm{S}_{0}$ geometries using state-averaged CASSCF (SA-CASSCF), with energy corrections from complete active space second-order perturbation theory (CASPT2), ${ }^{14}$ it was found that $S_{1}$ is a dark state with negligible oscillator strength and $S_{2}$ a bright $\pi \pi^{*}$ state populated by a UV photon (see Table $\mathrm{S} 2$ of the Supporting Information (SI)). Starting from the vertical $\mathrm{S}_{2}$ Franck-Condon (FC) points, the $E \rightarrow Z$ and $Z \rightarrow E$ photoisomerizations of 1 were then first modeled by performing MEP calculations at the SA-CASSCF level, as further described in the SI. The resulting MEPs are given in Figure 1.

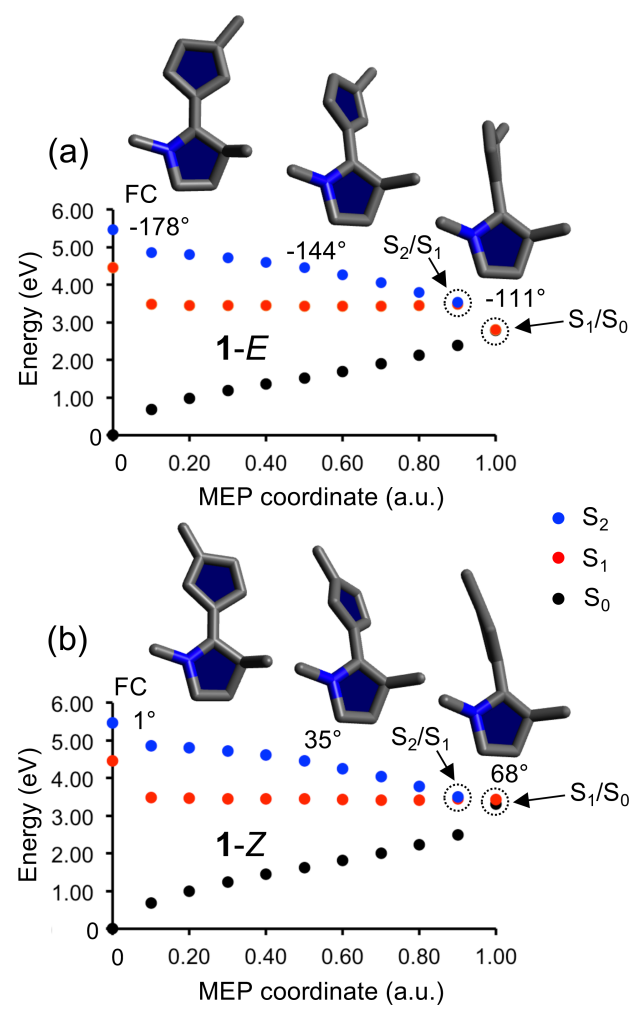

Figure 1. MEPs from the $\mathrm{S}_{2} \mathrm{FC}$ points of the $E$ (a) and $Z$ (b) isomers of motor 1 . Shown are also the motor geometries at the FC point and at two additional points along the respective path, as well as the corresponding $\omega$ dihedral angles (see Scheme 1). Encircled points are presumably close to conical intersection regions.

As illustrated by the MEPs, the geometric evolution in the $\mathrm{S}_{2}$ state is dominated by torsional motion around the central olefinic bond, which is barrierless and (not shown) facilitated by a $>0.1 \AA$ elongation of this bond. Through this motion, the systems approach an assumed $\mathrm{S}_{2} / \mathrm{S}_{1}$ conical intersection $(\mathrm{CI})$ region where they can decay to the $S_{1}$ state. With little further geometric distortion, the systems are then in a similar fashion funneled to the $\mathrm{S}_{0}$ state through an assumed $\mathrm{S}_{1} / \mathrm{S}_{0}$ CI. Importantly, the direction of torsional motion is the same for the $E$ and $Z$ isomers - toward increasing values of the $\omega$ dihedral angle (see Scheme 1), which is here defined as CCW motion. Furthermore, starting CASSCF $\mathrm{S}_{0}$ geometry optimizations from the end points of the MEPs, the relaxation following the $\mathrm{S}_{1} \rightarrow \mathrm{S}_{0}$ decay continues the CCW torsional motion and yields $1-Z$ as the photoproduct of $1-E$ and $1-E$ as the photoproduct of $1-Z$, respectively. Altogether, then, the MEP results predict that consecutive $E \rightarrow Z$ and $Z \rightarrow E$ photoisomerizations of 1 produce a full $360^{\circ}$ rotation, and thus that $\mathbf{1}$ is a light-driven rotary molecular motor that requires no thermal steps.

As for the character of the photoactive $S_{2}$ state of $\mathbf{1}$, Table $\mathrm{S} 4$ of the SI gives the net charges of the cyclopentadiene motif in the $\mathrm{S}_{0}, \mathrm{~S}_{1}$ and $\mathrm{S}_{2}$ states along the MEPs. Notably, despite that the motor is uncharged, in $\mathrm{S}_{2}$ this motif acquires a sizable amount of negative charge $(\sim 0.4-0.5 \mathrm{e})$, which indicates the (partial) formation of a cyclopentadienyl anion. Given that this anion is well known to be aromatic, it appears that part of the motor exhibits cyclic electron delocalization in the $S_{2}$ state. Corroborating this conclusion are two observations from Figure S2 and Table S5 of the SI regarding the motor geometries along the MEPs. First, from Figure S2, it can be seen that the cyclopentadiene bond length alternation (BLA) is reduced by $0.08-0.12 \AA$ in the $S_{2}$ state, compared to the situation for the FC geometries. Second, given that carbanions adopt distinctly pyramidal geometries in the absence of electron delocalization, ${ }^{15}$ it is notable from Table $\mathrm{S} 5$ that the $\mathrm{C} 1$ ' atom of the cyclopentadiene motif barely shows any pyramidalization at all.

With these results in mind and before probing the excitedstate aromaticity of $\mathbf{1}$ in more detailed terms below, it is of interest to investigate how this feature influences the photoisomerization dynamics of the motor. To this end, the $E \rightarrow Z$ and $Z \rightarrow E$ photoisomerizations of $\mathbf{1}$ were modeled by performing NAMD simulations. For comparison, such simulations were also carried out for an isoelectronic analogue of $\mathbf{1}$ denoted motor 2 (see Figure 2), wherein the cyclopentadiene motif is replaced by cyclopentene and, consequently, the possibility of excited-state aromaticity is lost. Importantly, through MEP calculations analogous to those performed for $\mathbf{1}$, but with the SA-CASSCF treatment adopted to the fact that the photoactive state of 2 is $S_{1}$ rather than $S_{2}$ (see Table S6 of the SI), it was first confirmed that also the UV-induced $E \rightarrow Z$ and $Z \rightarrow E$ photoisomerizations of 2 afford barrierless $360^{\circ}$ unidirectional rotary motion (see Figure S3 of the SI).

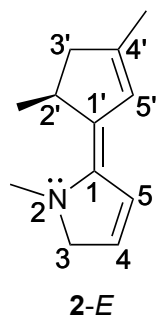

Figure 2. Chemical structure of the $E$ isomer of motor 2 .

As further described in the SI, the NAMD simulations were started in the photoactive state $\left(S_{2}\right.$ for motor $1 ; S_{1}$ for motor 2$)$ and were run at the SA-CASSCF/6-31G(d) level for maximally 800 fs and with 200 different initial nuclear configurations and velocities for both the $E$ and $Z$ isomers. Hops between states were allowed based on the magnitudes of the energy gap and non-adiabatic coupling between the states. ${ }^{9 a, 10}$ To quantify the efficiency of the motors, the rotary QY of a photoisomerization is defined as the percentage of the 200 trajectories that form the $Z(E)$ isomer from the $E(Z)$ isomer by completing a 
net $\mathrm{CCW} 180^{\circ}$ rotation around the central olefinic bond relative to the starting nuclear configuration within 800 fs. Furthermore, the photoisomerization time (PIT) is defined as the time needed for one such rotation, and the excited-state lifetime $(\tau)$ as the time needed for any trajectory rotating in the $\mathrm{CCW}$ direction to first reach the $\mathrm{S}_{0}$ state. The distributions of PIT and $\tau$ values from the NAMD simulations are presented in Figure 3 for motor 1 and in Figure S4 of the SI for motor 2. Shown are also the corresponding rotary QYs and the percentages of trajectories that reach the $\mathrm{S}_{0}$ state.
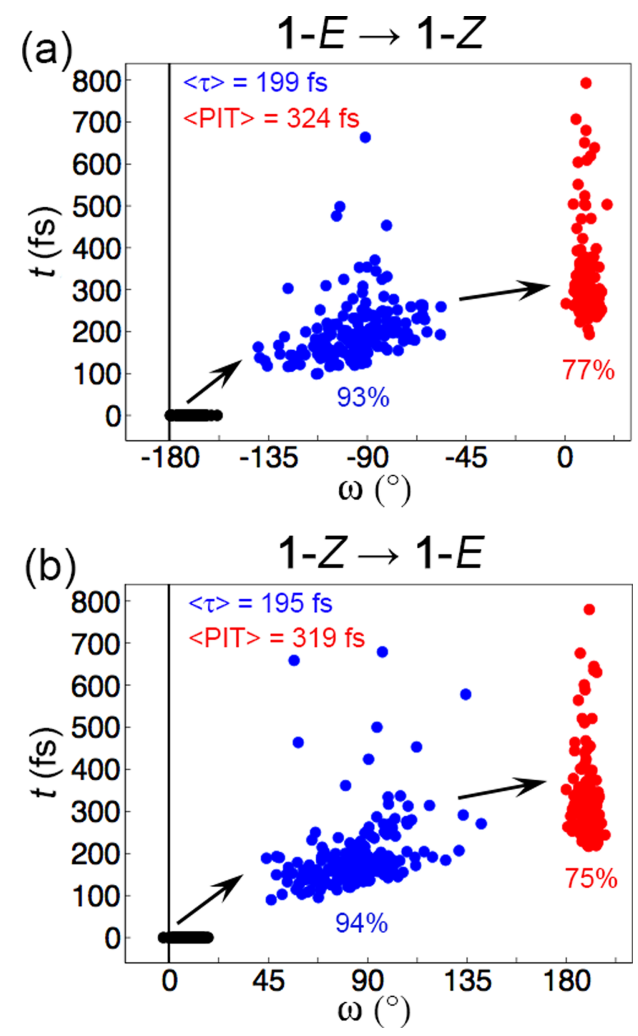

Figure 3. Distributions of $\tau$ (blue circles) and PIT (red circles) values for the $E \rightarrow Z$ (a) and $Z \rightarrow E$ (b) trajectories of motor 1 and the corresponding changes in the $\omega$ dihedral angle relative to the starting nuclear configurations (black circles). Shown are also the average $\tau$ and PIT values, the percentages of trajectories that reach the $\mathrm{S}_{0}$ state, and the rotary QYs.

Starting with Figure 3, it is notable that the rotary QYs of 1 are much higher, 77 and $75 \%$, than the QYs of $~ 20-30 \%$ typically achieved by overcrowded-alkene motors. ${ }^{4 a, c}$ Accordingly, the net CCW directionality of the full $360^{\circ}$ rotary cycle is a substantial $58 \%(77 \% \times 75 \%)$. Another positive feature of $\mathbf{1}$ is that the average $\tau$ and PIT values are only $\sim 200$ and $\sim 320 \mathrm{fs}$, respectively. As a comparison, overcrowded-alkene motors typically have excited-state lifetimes of $\sim 1$ ps or more. .a,c $^{4}$ Overall, it is also very encouraging that the performance data on 1 in Figure 3 compare very well with the corresponding data available for Schiff-base motors, ${ }^{8}$ despite that 1 lacks the ability of Schiff-base motors to favorably influence the efficiency of the rotary motion through a cationic nitrogen center. ${ }^{5}$

Continuing with the results for reference motor 2 in Figure $\mathrm{S} 4$, it is clear that replacing the cyclopentadiene motif of 1 with cyclopentene in $\mathbf{2}$ - and thereby foregoing the possibility of excited-state aromaticity - worsens the photochemical per- formance. In fact, this reduces the rotary QYs from 77 and $75 \%$ to 40 and $49 \%$, and increases the average $\tau$ and PIT values by $\sim 250$ fs. Thus, the presumed excited-state aromaticity of 1 has a major positive effect on the efficiency of this motor. This conclusion is corroborated by complementary NAMD results (summarized in the SI in connection to Figure S5) on a second isoelectronic reference motor, which, contrary to $\mathbf{2}$, maintains a methyl group at the $\mathrm{C} 5$ position.

In order to further probe the aromaticity of the cyclopentadiene motif in the $S_{2}$ state of $\mathbf{1}$, two different aromaticity indices were calculated based on the SA-CASSCF wave functions and geometries along the photoisomerization MEPs of 1: the Shannon aromaticity $(\mathrm{SA})^{16}$ index and the harmonic oscillator model of aromaticity (HOMA) ${ }^{17}$ index. As outlined in the SI, $\mathrm{SA}$ is an electronic index based on Bader's theory of atoms in molecules ${ }^{18}$ that probes the electron density variation at bond critical points (BCPs). ${ }^{16}$ HOMA, in turn, is a geometric index based on the deviation of the carbon-carbon bond lengths from an ideal aromatic reference value. ${ }^{17}$ The results of the calculations are given in Figure 4.
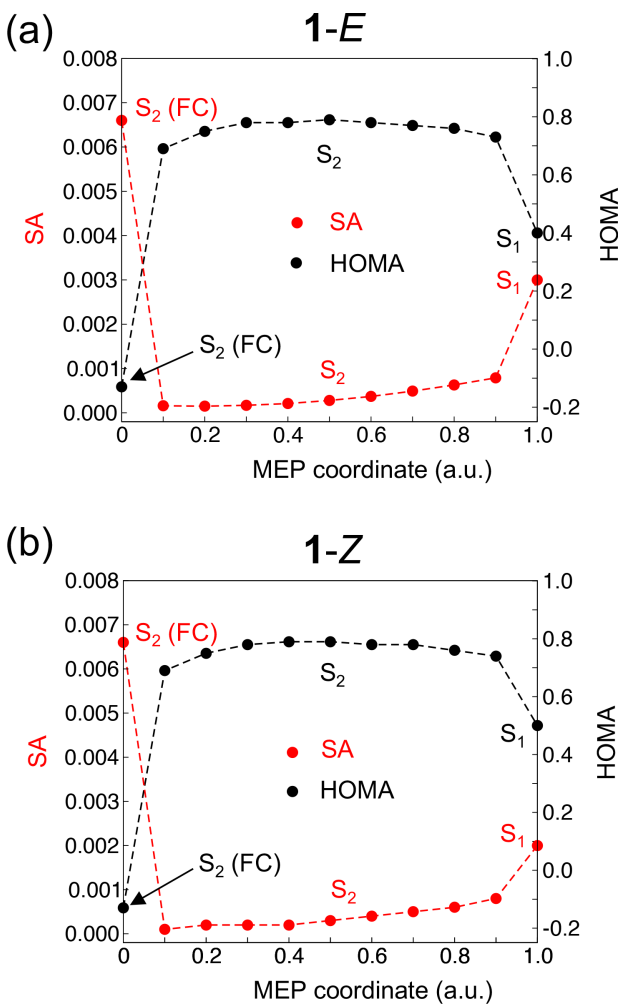

Figure 4. SA and HOMA values for the cyclopentadiene motif along the photoisomerization MEPs of the $E$ (a) and $Z$ (b) isomers of motor 1.

As can be seen from Figure 4, the SA values are $\sim 0.007$ at the $\mathrm{S}_{2}$ FC points but drop into the range of $0.0001-0.001$ as the photoisomerizations proceed in the $S_{2}$ state. Through a comparison with the previous BLA plots in Figure S2, it is clear that this effect is due to the pronounced cyclopentadiene bond length equalization in this state of the motor. The small SA values are reflective of small variations in electron density between different BCPs, as expected for an aromatic system. ${ }^{16}$ Moreover, the appreciable lowering of the SA values suggests that excited-state aromaticity may in fact provide the driving force for the photoisomerizations. The same picture emerges 
from consideration of the HOMA values, which are close to 0 at the $\mathrm{S}_{2} \mathrm{FC}$ points but subsequently increase into a range $(0.7-0.8)$ quite close to 1 , as usually found for aromatic systems. ${ }^{17 \mathrm{c}}$ Finally, as a qualitative validation of the results in Figure 4, Table S7 of the SI gives nucleus-independent chemical shifts ${ }^{19}$ of the $E$ and $Z$ isomers of $\mathbf{1}$ calculated at the $\mathrm{S}_{2} \mathrm{FC}$ point and a subsequent $S_{2}$ MEP point.

In summary, we have discovered a new route to the design of fast and efficient light-driven rotary molecular motors along which excited-state aromaticity is exploited to both shorten the lifetimes and increase the rotary QYs of the $Z / E$ photoisomerizations that underlie the motor function. Illustrating the potential of the route through comparative NAMD simulations of two motors with and without a moiety exhibiting such electron delocalization, the results attribute a key role to excited-state aromaticity in the future development of more powerful and efficient molecular motors.

\section{ASSOCIATED CONTENT}

\section{Supporting Information}

The Supporting Information is available free of charge on the ACS Publications website.

Computational details, complementary results (Figures S1-S5 and Tables S1-S7), description of multimedia files, and Cartesian coordinates and energies of different geometries of motors $\mathbf{1}$ and $\mathbf{2}$ (PDF). Two multimedia files are also supplied (AVI).

\section{AUTHOR INFORMATION}

\section{Corresponding Author}

*B.D.: E-mail: bodur@ifm.liu.se.

*J.W.: E-mail: jun.wang@liu.se.

\section{Notes}

The authors declare no competing financial interest.

\section{ACKNOWLEDGMENT}

We acknowledge financial support from the Swedish Research Council (grant 621-2011-4353), the Olle Engkvist Foundation (grant 2014/734), the Carl Trygger Foundation (grant CTS 15:134) and Linköping University, as well as grants of computing time at the National Supercomputer Centre (NSC) in Linköping.

\section{REFERENCES}

(1) (a) Koumura, N.; Zijlstra, R. W. J.; van Delden, R. A.; Harada, N.; Feringa, B. L. Nature 1999, 401, 152-155. (b) Koumura, N.; Geertsema, E. M.; van Gelder, M. B.; Meetsma, A.; Feringa, B. L. J. Am. Chem. Soc. 2002, 124, 5037-5051. (c) Pollard, M. M.; Klok, M.; Pijper, D.; Feringa, B. L. Adv. Funct. Mater. 2007, 17, 718-729. (d) Ruangsupapichat, N.; Pollard, M. M.; Harutyunyan, S. R.; Feringa, B. L. Nat. Chem. 2011, 3, 53-60. (e) Vachon, J.; Carroll, G. T.; Pollard, M. M.; Mes, E. M.; Brouwer, A. M.; Feringa, B. L. Photochem. Photobiol. Sci. 2014, 13, 241-246.

(2) (a) Chiang, P.-T.; Mielke, J.; Godoy, J.; Guerrero, J. M.; Alemany, L. B.; Villagómez, C. J.; Saywell, A.; Grill, L.; Tour, J. M. ACS Nano 2012, 6, 592-597. (b) Chen, J.; Wezenberg, S. J.; Feringa, B. L. Chem. Commun. 2016, 52, 6765-6768. (c) Saywell, A.; Bakker, A.; Mielke, J.; Kumagai, T.; Wolf, M.; García-López, V.; Chiang, P.-T.; Tour, J. M.; Grill, L. ACS Nano 2016, 10, 10945-10952.

(3) (a) van Dijken, D. J.; Chen, J.; Stuart, M. C. A.; Hou, L.; Feringa, B. L. J. Am. Chem. Soc. 2016, 138, 660-669. (b) Zhao, D.; van Leeuwen, T.; Cheng, J.; Feringa, B. L. Nat. Chem. 2017, 9, 250-256.
(4) (a) Kazaryan, A.; Lan, Z.; Schäfer, L. V.; Thiel, W.; Filatov, M. $J$. Chem. Theory Comput. 2011, 7, 2189-2199. (b) Conyard, J.; Addison, K.; Heisler, I. A.; Cnossen, A.; Browne, W. R.; Feringa, B. L.; Meech, S. R. Nat. Chem. 2012, 4, 547-551. (c) Conyard, J.; Cnossen, A.; Browne, W. R.; Feringa, B. L.; Meech, S. R. J. Am. Chem. Soc. 2014, 136, 9692-9700.

(5) Filatov, M.; Olivucci, M. J. Org. Chem. 2014, 79, 3587-3600.

(6) (a) Pérez-Hernández, G.; González, L. Phys. Chem. Chem. Phys. 2010, 12, 12279-12289. (b) Oruganti, B.; Fang, C.; Durbeej, B. Phys. Chem. Chem. Phys. 2015, 17, 21740-21751. (c) Oruganti, B.; Wang, J.; Durbeej, B. ChemPhysChem 2016, 17, 3399-3408.

(7) (a) Amatatsu, Y. J. Phys. Chem. A 2012, 116, 10182-10193. (b) García-Iriepa, C.; Marazzi, M.; Zapata, F.; Valentini, A.; Sampedro, D.; Frutos, L. M. J. Phys. Chem. Lett. 2013, 4, 1389-1396. (c) Marchand, G.; Eng, J.; Schapiro, I.; Valentini, A.; Frutos, L. M.; Pieri, E.; Olivucci, M.; Léonard, J.; Gindensperger, E. J. Phys. Chem. Lett. 2015, 6, 599-604. (d) Greb, L.; Eichhöfer, A.; Lehn, J.-M. Angew. Chem. Int. Ed. 2015, 54, 14345-14348.

(8) (a) Nikiforov, A.; Gamez, J. A.; Thiel, W.; Filatov, M. J. Phys. Chem. Lett. 2016, 7, 105-110. (b) Wang, J.; Oruganti, B.; Durbeej, B. Phys. Chem. Chem. Phys. 2017, 19, 6952-6956.

(9) (a) Groenhof, G.; Bouxin-Cademartory, M.; Hess, B.; de Visser, S. P.; Berendsen, H. J. C.; Olivucci, M.; Mark, A. E.; Robb, M. A. J. Am. Chem. Soc. 2004, 126, 4228-4233. (b) Barbatti, M. WIREs Comput. Mol. Sci. 2011, 1, 620-633. (c) Fdez. Galván, I.; Delcey, M. G.; Pedersen, T. B.; Aquilante, F.; Lindh, R. J. Chem. Theory. Comput. 2016, 12, 3636-3653. (d) Liu, L.; Liu, J.; Martinez, T. J. J. Phys. Chem. B 2016, 120, 1940-1949.

(10) Aquilante, F.; Autschbach, J.; Carlson, R. K.; Chibotaru, L. F.; Delcey, M. G.; De Vico, L.; Fdez. Galván, I.; Ferré, N.; Frutos, L. M.; Gagliardi, L.; Garavelli, M.; Giussani, A.; Hoyer, C. E.; Manni, G. L.; Lischka, H.; Ma, D.; Malmqvist, P.-Å.; Müller, T.; Nenov, A.; Olivucci, M.; Pedersen, T. B.; Peng, D.; Plasser, F.; Pritchard, B.; Reiher, M.; Rivalta, I.; Schapiro, I.; Segarra-Martí, J.; Stenrup, M.; Truhlar, D. G.; Ungur, L.; Valentini, A.; Vancoillie, S.; Veryazov, V.; Vysotskiy, V. P.; Weingart, O.; Zapata, F.; Lindh, R. J. Comput. Chem. 2016, 37, 506-541.

(11) (a) Ottosson, H. Nat. Chem. 2012, 4, 969-971. (b) Rosenberg, M.; Dahlstrand, C.; Kilså, K.; Ottosson, H. Chem. Rev. 2014, 114, 5379-5425. (c) Sung, Y. M.; Oh, J.; Kim, W.; Mori, H.; Osuka, A.; Kim, D. J. Am. Chem. Soc. 2015, 137, 11856-11859.

(12) (a) Mohamed, R. K.; Mondal, S.; Jorner, K.; Delgado, T. F.; Lobodin, V. V.; Ottosson, H.; Alabugin, I. V. J. Am. Chem. Soc. 2015, 137, 15441-15450. (b) Papadakis, R.; Li, H.; Bergman, J.; Lundstedt, A.; Jorner, K.; Ayub, R.; Haldar, S.; Jahn, B. O.; Denisova, A.; Zietz, B.; Lindh, R.; Sanyal, B.; Grennberg, H.; Leifer, K.; Ottosson, H. Nat. Commun. 2016, 7, 12962:1-10.

(13) Roos, B. O.; Taylor, P. R.; Siegbahn, P. E. M. Chem. Phys. 1980, $48,157-173$.

(14) (a) Andersson, K.; Malmqvist, P.-Å.; Roos, B. O. J. Chem. Phys. 1992, 96, 1218-1226. (b) Finley, J.; Malmqvist, P.-Å.; Roos, B. O.; Serrano-Andrés, L. Chem. Phys. Lett. 1998, 288, 299-306.

(15) (a) Wiberg, K. B.; Castejon, H. J. Am. Chem. Soc. 1994, 116, 10489-10497. (b) Wiberg, K. B.; Castejon, H. J. Org. Chem. 1995, $60,6327-6334$.

(16) (a) Noorizadeh, S.; Shakerzadeh, E. Phys. Chem. Chem. Phys. 2010, 12, 4742-4749. (b) Noorizadeh, S.; Shakerzadeh, E. Comput. Theo. Chem. 2011, 964, 141-147.

(17) (a) Kruszewski, J.; Krygowki, T. M. Tetrahedron Lett. 1972, 13, 3839-3842. (b) Krygowki, T. M. J. Chem. Inf. Comput. Sci. 1993, 33, 70-78. (c) Krygowski, T. M.; Cyrański, M. K. Chem. Rev. 2001, 101, $1385-1419$.

(18) Bader, R. F. W. Chem. Rev. 1991, 91, 893-928.

(19) (a) von Ragué Schleyer, P.; Maerker, C.; Dransfeld, A.; Jiao, H.; van Eikema Hommes, N. J. R. J. Am. Chem. Soc. 1996, 118, 63176318. (b) Karadakov, P. B. J. Phys. Chem. A 2008, 112, 7303-7309. 


\section{Excited-State Aromaticity Improves Molecular Motors: A Computational Analysis}

Baswanth Oruganti, Jun Wang* and Bo Durbeej*

Division of Theoretical Chemistry, IFM, Linköping University, SE-581 83 Linköping, Sweden

*B.D.: E-mail: bodur@ifm.liu.se

*J.W.: E-mail: jun.wang@liu.se

Supporting Information 


\section{Table of contents}

Computational details

Figure S1

Figure S2

Figure S3

Figure S4

Figure S5

Table S1

Table S2

Table S3

Table S4

Table S5

Table S6

Table S7

Description of multimedia files

References for the Supporting Information

Cartesian coordinates and energies of different geometries of motors $\mathbf{1}$ and $\mathbf{2}$ pages S3-S6

page $\mathrm{S} 7$

page $\mathrm{S} 8$

page $\mathrm{S} 9$

page $\mathrm{S} 10$

page $\mathrm{S} 11$

page $\mathrm{S} 12$

page $\mathrm{S} 13$

page $\mathrm{S} 14$

page $\mathrm{S} 15$

page $\mathrm{S} 16$

page $\mathrm{S} 17$

page $\mathrm{S} 18$

page S19

pages S20-S21

pages S22-S70 


\section{Computational details}

MEP calculations. The minimum energy path (MEP) calculations on motor 1 were carried out with the complete active space self-consistent field (CASSCF) method $^{1}$ in combination with Dunning's correlation-consistent polarized valence triple- $\zeta$ (cc-pVTZ) basis set. ${ }^{2}$ The active space included eight electrons (six $\pi$ and the nitrogen lone pair) distributed in seven orbitals. First, following CASSCF optimization of the ground-state $\left(\mathrm{S}_{0}\right)$ equilibrium geometries of the $E$ and $Z$ isomers, the wave functions at the vertical Franck-Condon (FC) points in the first $\left(\mathrm{S}_{1}\right)$ and second $\left(\mathrm{S}_{2}\right)$ excited singlet states of the two isomers were calculated using state-averaged CASSCF (SA-CASSCF) with equal weights for the $S_{0}, S_{1}$ and $S_{2}$ states and with energy corrections from complete active space second-order perturbation theory (CASPT2). ${ }^{3}$ Having found from these calculations that $\mathrm{S}_{1}$ is a dark state with negligible oscillator strength and $\mathrm{S}_{2}$ a bright $\pi \pi^{*}$ state populated by absorption of a UV photon (see Table S2), the actual SA-CASSCF MEP calculations were then started from the $\mathrm{S}_{2} \mathrm{FC}$ points.

With one exception, the protocol just described was also employed for the MEP calculations on motor 2. Specifically, since the photoactive state of $\mathbf{2}$ is $S_{1}$ rather than $S_{2}$ (see Table $S 6$ ), the corresponding SA-CASSCF treatment included only the $\mathrm{S}_{0}$ and $\mathrm{S}_{1}$ states, with equal weights.

NAMD simulations. Similar to the MEP calculations, the non-adiabatic molecular dynamics (NAMD) simulations of motor 1 were also carried out using three-state $\left(\mathrm{S}_{0}, \mathrm{~S}_{1}\right.$ and $\left.\mathrm{S}_{2}\right)$ SA-CASSCF with an active space of eight electrons in seven orbitals. For all simulations, Pople's 6-31G(d) splitvalence double- $\zeta$ plus polarization basis $\operatorname{set}^{4}$ was used. First, following calculation of the $\mathrm{S}_{0}$ vibrational normal modes of the $E$ and $Z$ isomers by means of Møller-Plesset second-order perturbation theory (MP2) with the def2-SVP basis set $^{5}$ and within the resolution-of-the-identity (RI) approximation, ${ }^{6} 200$ different initial nuclear configurations and velocities for the two isomers were generated from a harmonic-oscillator Wigner distribution. ${ }^{7}$ The use of MP2 for calculating the normal modes was validated through a favorable comparison of the geometries obtained with this method and those obtained with CASPT2 (see Tables S2 and S3). For both isomers, 200 photoisomerization trajectories were then initiated by promoting the systems to the $\mathrm{S}_{2}$ state. Evaluating nuclear forces "on the fly", the trajectories were propagated classically using the velocity Verlet algorithm ${ }^{8}$ with a fixed integration time step of $0.5 \mathrm{fs}$. 
Employing the algorithm by Robb and co-workers ${ }^{9}$ as implemented in the MOLCAS 8.0 suite of programs, ${ }^{10}$ the non-adiabatic coupling vectors $\left\langle\Psi_{i} \mid \frac{\partial}{\partial \mathrm{R}} \Psi_{j}\right\rangle$ between the $\mathrm{S}_{2}$ and $\mathrm{S}_{1}$ states and between the $\mathrm{S}_{1}$ and $\mathrm{S}_{0}$ states were evaluated in the framework of Landau-Zener theory, ${ }^{11}$ wherein the couplings are large when $\left\langle\Psi_{i} \mid \frac{\partial}{\partial t} \Psi_{j}\right\rangle$ is large. Specifically, the $\left\langle\Psi_{i} \mid \frac{\partial}{\partial t} \Psi_{j}\right\rangle$ terms were computed at each step along the trajectories for which the energy gap between the states is smaller than 0.03 a.u., using the numerical approximation

$$
\left\langle\Psi_{i}(t) \mid \frac{\partial}{\partial t} \Psi_{j}(t)\right\rangle \approx \frac{\left\langle\Psi_{i}(t) \mid \Psi_{j}(t+\Delta t)\right\rangle}{\Delta t} .
$$

Based on this model, a trajectory was allowed to hop from one state to the other (and later possibly back again) when $\left\langle\Psi_{i}(t) \mid \Psi_{j}(t+\Delta t)\right\rangle$ is larger than 0.25 , representing situations when the wave functions of the states in questions begin to deviate from orthogonality close to a conical intersection. Allowing maximally 20 hops in each trajectory, the simulations were run for up to 800 fs.

The NAMD simulations of motor $\mathbf{2}$, in turn, were carried out with the same exact protocol as those of motor 1, expect that the trajectories were started in the $S_{1}$ state and were run using twostate $\left(\mathrm{S}_{0}\right.$ and $\left.\mathrm{S}_{1}\right)$ SA-CASSCF, to account for the fact that $\mathrm{S}_{1}$ rather than $\mathrm{S}_{2}$ is the photoactive state of this system (see Table S6).

Calculation of aromaticity indices. Below, the procedures to calculate Shannon aromaticity (SA) ${ }^{12}$ and harmonic oscillator model of aromaticity (HOMA) ${ }^{13}$ indices are described. Based on SACASSCF wave functions and geometries along the photoisomerization MEPs of motor $\mathbf{1}$, these indices were used to probe the aromaticity of the cyclopentadiene motif in the $S_{2}$ state of motor 1 .

Starting with SA, this electronic index is based on Bader's theory of atoms in molecules $(\mathrm{AIM})^{14}$ and probes the variation in electron density between different bond critical points (BCPs) of the aromatic ring in question. ${ }^{12}$ Specifically, this index is formulated in terms of the so-called Shannon entropy, ${ }^{15}$ defined as

$$
S=-\sum_{i}^{N} p_{i}\left(\mathbf{r}_{\mathrm{c}}\right) \ln p_{i}\left(\mathbf{r}_{\mathrm{c}}\right)
$$


where $\mathbf{r}_{\mathrm{c}}$ is a BCP of the ring and $N$ is the number of BCPs in the ring. Moreover, $p_{i}\left(\mathbf{r}_{\mathrm{c}}\right)$ is the normalized probability electron density at a given BCP, defined as

$$
p_{i}\left(\mathbf{r}_{\mathrm{c}}\right)=\frac{\rho_{i}\left(\mathbf{r}_{\mathrm{c}}\right)}{\sum_{i}^{N} \rho_{i}\left(\mathbf{r}_{\mathrm{c}}\right)}
$$

where $\rho_{i}\left(\mathbf{r}_{\mathrm{c}}\right)$ is the electron density at that BCP. By defining the SA index as the difference between the Shannon entropy that the system under consideration would have if it was perfectly aromatic and the Shannon entropy that it actual has, a small value for this difference then suggests that the electron density variation at BCPs is minor, as expected for an aromatic system. ${ }^{12}$ Typically, aromatic compounds show SA values below $0.003 .^{12}$

Continuing with HOMA, this geometric index probes the deviation of the carbon-carbon bond lengths $R_{i}$ of the aromatic ring in question from the reference optimal bond length $R_{\mathrm{opt}}$ of the fully aromatic benzene molecule. ${ }^{13}$ Specifically,

$$
\mathrm{HOMA}=1-\frac{\alpha}{n} \sum_{i}^{n}\left(R_{i}-R_{\mathrm{opt}}\right)^{2},
$$

where $n$ is the number of carbon-carbon bonds and $\alpha$ is an empirical normalization factor chosen in such a way that HOMA approaches 1 for an aromatic compound with all $R_{i}$ close to $R_{\text {opt }}$, and approaches 0 for the corresponding (and hypothetical) non-aromatic Kekule structures. ${ }^{13}$ In this work, the standard parameters $\alpha=257.7 \AA^{-2}$ and $R_{\text {opt }}=1.388 \AA$ were employed. ${ }^{13}$ Although these parameters have been defined for studies of ground-state compounds, this does not prevent them from being used for a balanced comparison of HOMA values for a series of different excited-state geometries of one single compound, for which the parameters are equally appropriate.

Finally, as a complement to the SA and HOMA indices, nucleus-independent chemical shifts (NICSs) ${ }^{16}$ of the $E$ and $Z$ isomers of motor 1 were calculated at the $\mathrm{S}_{2}$ FC point and an arbitrarily chosen (the fifth) subsequent $\mathrm{S}_{2}$ MEP point. Specifically, at these points, NICS(1) ZZ values corresponding to the negative of the ZZ-component of the magnetic shielding tensor $1 \AA$ above the cyclopentadiene ring center (as obtained from AIM theory) were calculated from statespecific CASSCF $(8,7)$ wave functions obtained using gauge-including atomic orbitals and the 6$31 \mathrm{G}(\mathrm{d})$ basis set. For NICSs, negative values indicate aromaticity. ${ }^{16 \mathrm{a}}$ The reason for limiting the 
NICS calculations to a single $\mathrm{S}_{2}$ MEP point is the technical requirement that the $\mathrm{S}_{2}$ CASSCF wave function is converged in a state-specific fashion, which becomes progressively more difficult as the $\mathrm{S}_{2}$ and $\mathrm{S}_{1}$ states come closer in energy.

Software used. The RI-MP2 calculations were done with TURBOMOLE $6.3 .{ }^{17}$ The SA and HOMA indices were calculated with Multiwfn. ${ }^{18}$ The NICS calculations were done with Dalton2013. ${ }^{19}$ All other calculations were done with MOLCAS 8.0. ${ }^{10}$ 


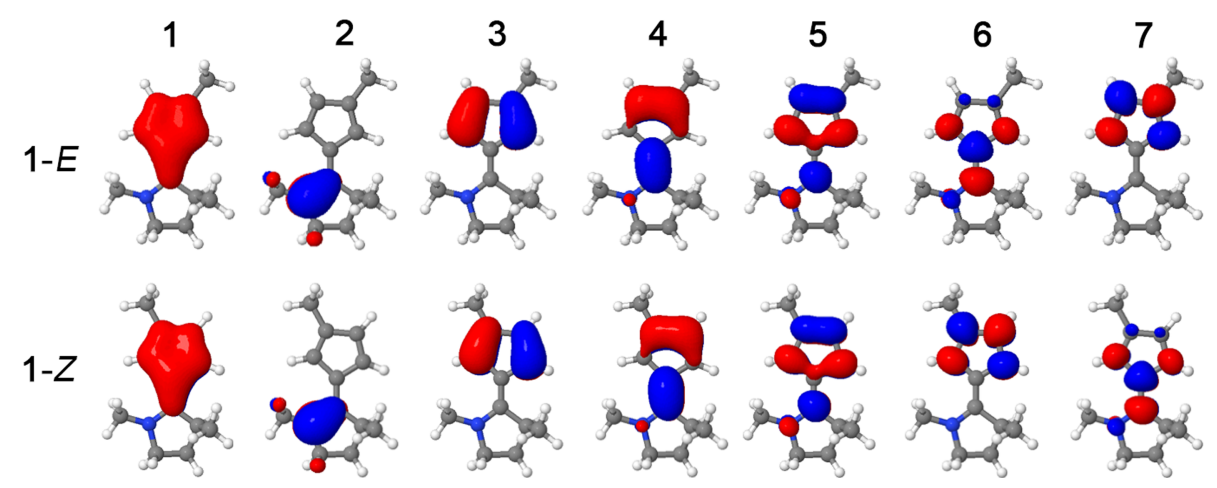

Figure S1. Active molecular orbitals at the CASSCF/cc-pVTZ $\mathrm{S}_{0}$ equilibrium geometries of the $E$ and $Z$ isomers of motor 1 . 

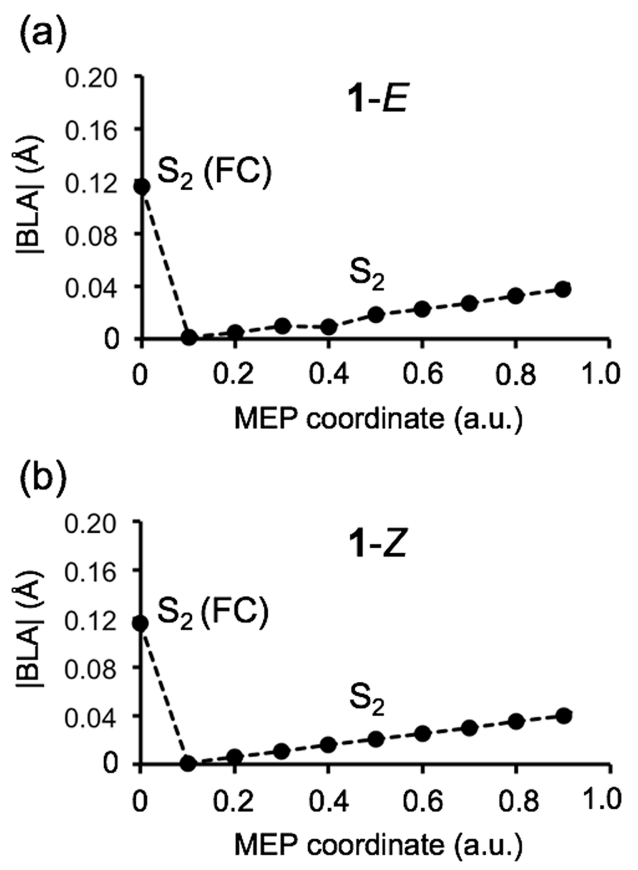

Figure S2. Bond length alternation (BLA) in the cyclopentadiene motif along the photoisomerization MEPs of the $E$ (a) and $Z$ (b) isomers of motor 1. Only MEP points in the $\mathrm{S}_{2}$ state are shown. 

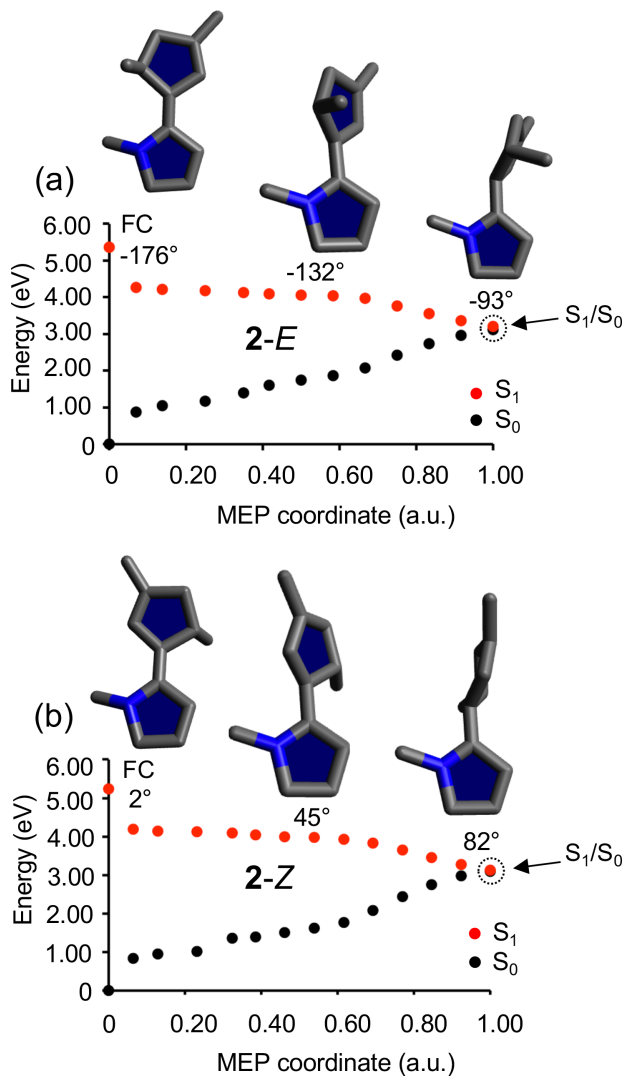

Figure S3. MEPs from the $\mathrm{S}_{1} \mathrm{FC}$ points of the $E$ (a) and $Z$ (b) isomers of motor 2. Shown are also the motor geometries at the FC point and at two additional points along the respective path, as well as the corresponding $\omega$ dihedral angles (see Scheme 1). Encircled points are presumably close to conical intersection regions. 

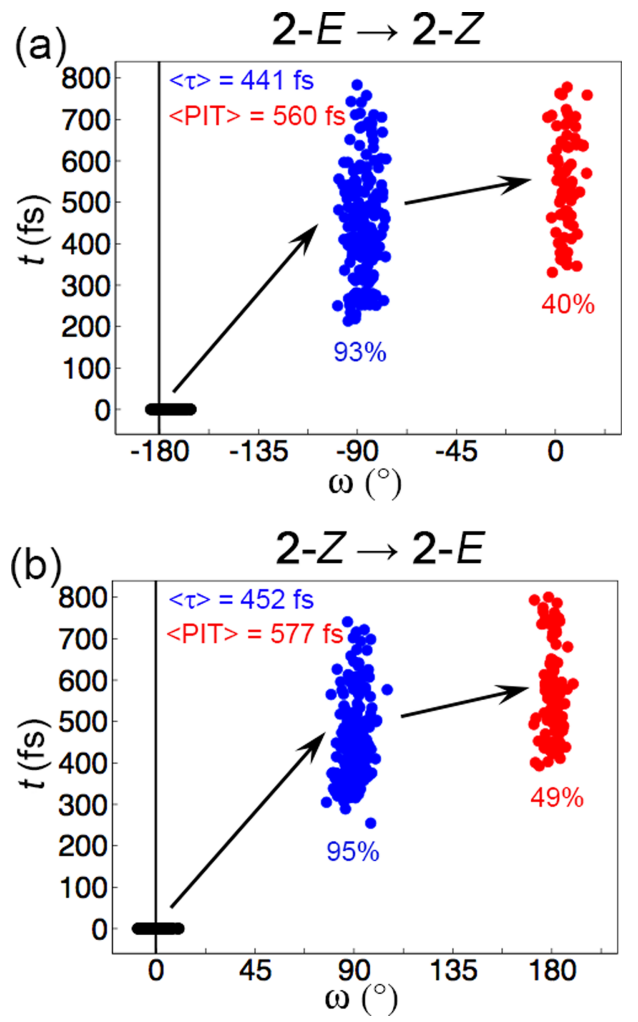

Figure S4. Distributions of excited-state lifetimes ( $\tau$, blue circles) and photoisomerization times (PITs, red circles) for the $E \rightarrow Z$ (a) and $Z \rightarrow E$ (b) trajectories of motor 2 and the corresponding changes in the $\omega$ dihedral angle relative to the starting nuclear configurations (black circles). Shown are also the average $\tau$ and PIT values, the percentages of trajectories that reach the $\mathrm{S}_{0}$ state, and the rotary QYs. 


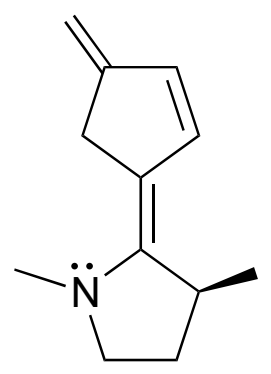

\section{3-E}

Figure S5. Chemical structure of the $E$ isomer of motor 3.

Comments on Figure S5: Just like reference motor 2, $\mathbf{3}$ is an isoelectronic analogue of motor 1, that was studied to further solidify the conclusion that excited-state aromaticity is a key factor for the efficiency of $\mathbf{1}$. Similar to 2, 3 cannot exhibit excited-state aromaticity. However, in contrast to 2, 3 carries a methyl group at the C5 position, which is also the situation for 1. First, through CASSCFbased calculations identical to those summarized for $\mathbf{2}$ in Table S6, it was found that the photoactive state of 3 is $S_{1}$, with oscillator strengths of $\sim 0.4-0.5$. Second, in NAMD simulations of 3 identical to those performed for $\mathbf{2}$ but with much fewer initial nuclear configurations and velocities considered (10 instead of 200), it was observed that 4 out of the 10 trajectories run for both the $E$ and $Z$ isomers of 3 complete a $180^{\circ}$ rotation within $800 \mathrm{fs}$, with the same preferred direction of rotation. Comparing with the 75/77\% rotary QYs of 1 (see Figure 2), these results underline the positive influence of excited-state aromaticity on the motor efficiency. Moreover, comparing with the 40/49\% rotary QYs of 2 (see Figure S4), they also suggest that the positions of methyl groups are not important in this regard. 
Table S1. Occupation Numbers of Active Molecular Orbitals in the $S_{0}, S_{1}$ and $S_{2}$ States of the $E$ and $Z$ Isomers of Motor $\mathbf{1}^{a}$

\begin{tabular}{llccccccc} 
& & \multicolumn{7}{c}{ active molecular orbital } \\
\cline { 3 - 9 } isomer & state & 1 & 2 & 3 & 4 & 5 & 6 & 7 \\
& $\mathrm{~S}_{0}$ & 1.96 & 2.00 & 1.91 & 1.91 & 0.11 & 0.05 & 0.06 \\
& $\mathrm{~S}_{1}$ & 1.95 & 2.00 & 1.89 & 1.05 & 0.97 & 0.06 & 0.08 \\
& $\mathrm{~S}_{2}$ & 1.95 & 1.96 & 1.32 & 1.86 & 0.69 & 0.07 & 0.15 \\
& $\mathrm{~S}_{0}$ & 1.96 & 2.00 & 1.91 & 1.91 & 0.11 & 0.05 & 0.06 \\
& $\mathrm{~S}_{1}$ & 1.95 & 2.00 & 1.89 & 1.03 & 0.99 & 0.06 & 0.08 \\
& $\mathrm{~S}_{2}$ & 1.95 & 1.96 & 1.32 & 1.86 & 0.70 & 0.07 & 0.15
\end{tabular}

${ }^{a}$ All calculations performed based on SA-CASSCF/cc-pVTZ wave functions computed with equal weights for the $\mathrm{S}_{0}, \mathrm{~S}_{1}$ and $\mathrm{S}_{2}$ states at CASSCF/cc-pVTZ $\mathrm{S}_{0}$ equilibrium geometries. 


\begin{tabular}{|c|c|c|c|c|c|c|}
\hline \multicolumn{7}{|c|}{ Table S2. Vertical CASPT2/cc-pVTZ Excitation Energies (eV) of the $E$ and $Z$ Isomers of Motor $1^{a}$} \\
\hline \multirow[b]{2}{*}{ isomer } & \multirow[b]{2}{*}{ excitation } & \multirow[b]{2}{*}{ CASPT2 treatment } & \multicolumn{4}{|c|}{$\mathrm{S}_{0}$ geometry } \\
\hline & & & $\begin{array}{l}\text { CASSCF/ } \\
\text { cc-pVTZ }\end{array}$ & $\begin{array}{l}\text { CASPT2/ } \\
\text { cc-pVDZ }^{b}\end{array}$ & $\begin{array}{l}\text { RI-MP2/ } \\
\text { cc-pVDZ }\end{array}$ & $\begin{array}{l}\text { RI-MP2/ } \\
\text { def2-SVP }\end{array}$ \\
\hline \multirow[t]{4}{*}{$E$} & \multirow[t]{2}{*}{$\mathrm{S}_{0} \rightarrow \mathrm{S}_{1}$} & SS-CASPT2 $^{c}$ & $4.03(0.014)$ & $3.74(0.029)$ & $3.75(0.023)$ & $3.74(0.030)$ \\
\hline & & MS-CASPT $^{d}$ & $4.14(0.014)$ & $3.87(0.029)$ & $3.87(0.023)$ & $3.87(0.030)$ \\
\hline & \multirow[t]{2}{*}{$\mathrm{S}_{0} \rightarrow \mathrm{S}_{2}$} & SS-CASPT $2^{c}$ & $4.05(0.691)$ & $3.94(0.535)$ & $4.06(0.529)$ & $3.95(0.541)$ \\
\hline & & MS-CASPT2 $^{d}$ & $4.32(0.691)$ & $4.23(0.535)$ & $4.32(0.529)$ & $4.24(0.541)$ \\
\hline \multirow[t]{4}{*}{$Z$} & \multirow[t]{2}{*}{$\mathrm{S}_{0} \rightarrow \mathrm{S}_{1}$} & SS-CASPT2 $^{c}$ & $4.05(0.013)$ & $3.75(0.028)$ & $3.75(0.023)$ & $3.75(0.030)$ \\
\hline & & MS-CASPT $^{d}$ & $4.16(0.013)$ & $3.88(0.028)$ & $3.88(0.023)$ & $3.88(0.030)$ \\
\hline & \multirow[t]{2}{*}{$\mathrm{S}_{0} \rightarrow \mathrm{S}_{2}$} & SS-CASPT2 ${ }^{c}$ & $4.05(0.696)$ & $3.94(0.537)$ & $4.05(0.530)$ & $3.95(0.542)$ \\
\hline & & MS-CASPT2 $^{d}$ & $4.31(0.696)$ & $4.22(0.537)$ & $4.32(0.530)$ & $4.23(0.542)$ \\
\hline \multicolumn{7}{|c|}{$\begin{array}{l}{ }^{a} \text { All calculations performed based on SA-CASSCF wave functions computed with equal weights for the } \mathrm{S}_{0} \text {, } \\
\mathrm{S}_{1} \text { and } \mathrm{S}_{2} \text { states. Oscillator strengths in parentheses obtained with the complete active space state-interaction } \\
\text { (CASSI) method (Ref. 20). }{ }^{b} \text { Obtained with numerical CASPT2 gradients. }{ }^{c} \text { State-specific CASPT2. } \\
{ }^{d} \text { Multi-state CASPT2. }\end{array}$} \\
\hline
\end{tabular}




\begin{tabular}{|c|c|c|c|c|c|}
\hline isomer & parameter $^{a}$ & $\begin{array}{l}\text { CASSCF/ } \\
\text { cc-pVTZ }\end{array}$ & $\begin{array}{l}\text { CASPT2/ } \\
\text { cc-pVDZ }\end{array}$ & $\begin{array}{l}\text { RI-MP2/ } \\
\text { cc-pVDZ }\end{array}$ & $\begin{array}{l}\text { RI-MP2/ } \\
\text { def2-SVP }\end{array}$ \\
\hline \multirow[t]{5}{*}{$E$} & $\mathrm{C} 1-\mathrm{C} 1^{\prime}$ & 1.36 & 1.39 & 1.39 & 1.38 \\
\hline & $\mathrm{C} 1-\mathrm{N} 2$ & 1.37 & 1.36 & 1.36 & 1.35 \\
\hline & $\theta$ & -178 & -176 & -173 & -174 \\
\hline & $\theta^{\prime}$ & -179 & -171 & -169 & -168 \\
\hline & $\omega$ & -178 & -173 & -171 & -171 \\
\hline \multirow[t]{5}{*}{$Z$} & $\mathrm{C} 1-\mathrm{C} 1^{\prime}$ & 1.36 & 1.39 & 1.39 & 1.38 \\
\hline & $\mathrm{C} 1-\mathrm{N} 2$ & 1.37 & 1.36 & 1.36 & 1.35 \\
\hline & $\theta$ & 0 & 5 & 8 & 8 \\
\hline & $\theta^{\prime}$ & 2 & 7 & 10 & 10 \\
\hline & $\omega$ & 1 & 6 & 9 & 9 \\
\hline
\end{tabular}


Table S4. Sum of Mulliken Atomic Charges in the Cyclopentadiene Motif in the $S_{0}, S_{1}$ and $S_{2}$ States along the Photoisomerization MEPs of the $E$ and $Z$ Isomers of Motor $1^{a}$

\begin{tabular}{clccccccccccc}
\multicolumn{10}{c}{} & \multicolumn{10}{c}{ geometry/MEP point $^{b}$} \\
\cline { 2 - 13 } isomer & state & $\mathrm{FC}^{c}$ & $\mathrm{FC}$ & 1 & 2 & 3 & 4 & 5 & 6 & 7 & 8 & 9 \\
& $\mathrm{~S}_{0}$ & -0.16 & -0.17 & -0.22 & -0.21 & -0.21 & -0.22 & -0.23 & -0.24 & -0.25 & -0.25 & -0.25 \\
& $\mathrm{~S}_{1}$ & & 0.02 & 0.08 & 0.08 & 0.08 & 0.08 & 0.07 & 0.07 & 0.07 & 0.07 & 0.07 \\
& $\mathrm{~S}_{2}$ & & -0.41 & -0.37 & -0.41 & -0.43 & -0.45 & -0.46 & -0.47 & -0.47 & -0.47 & -0.47 \\
& $\mathrm{~S}_{0}$ & -0.14 & -0.15 & -0.20 & -0.20 & -0.20 & -0.20 & -0.21 & -0.22 & -0.23 & -0.23 & -0.24 \\
& $\mathrm{~S}_{1}$ & & 0.05 & 0.09 & 0.10 & 0.10 & 0.10 & 0.09 & 0.09 & 0.09 & 0.08 & 0.08 \\
& $\mathrm{~S}_{2}$ & & -0.39 & -0.36 & -0.39 & -0.42 & -0.43 & -0.44 & -0.45 & -0.45 & -0.45 & -0.45
\end{tabular}

${ }^{a}$ All calculations except where otherwise indicated performed based on SA-CASSCF/cc-pVTZ wave functions computed with equal weights for the $\mathrm{S}_{0}, \mathrm{~S}_{1}$ and $\mathrm{S}_{2}$ states. ${ }^{b}$ Only MEP points in the $\mathrm{S}_{2}$ state are shown. ${ }^{c}$ Calculations performed based on state-specific CASSCF/cc-pVTZ wave functions. 


\begin{tabular}{|c|c|c|c|c|c|c|c|c|c|c|c|}
\hline \multicolumn{12}{|c|}{$\begin{array}{l}\text { Table S5. Pyramidalizat } \\
\text { Isomers of Motor } 1^{a}\end{array}$} \\
\hline \multirow[b]{2}{*}{ isomer } & \multirow[b]{2}{*}{ angle $^{c}$} & \multicolumn{10}{|c|}{ geometry/MEP point ${ }^{b}$} \\
\hline & & $\mathrm{FC}$ & 1 & 2 & 3 & 4 & 5 & 6 & 7 & 8 & 9 \\
\hline \multirow[t]{2}{*}{ E } & $\alpha$ & 0 & -6 & -12 & -15 & -16 & -16 & -16 & -15 & -14 & -13 \\
\hline & $\alpha^{\prime}$ & 0 & 0 & 0 & 1 & 1 & 1 & 1 & 1 & 1 & 0 \\
\hline \multirow[t]{2}{*}{$Z$} & $\alpha$ & 0 & -6 & -13 & -16 & -17 & -18 & -18 & -18 & -17 & -16 \\
\hline & $\alpha^{\prime}$ & 1 & 0 & 1 & 2 & 3 & 4 & 5 & 6 & 6 & 7 \\
\hline
\end{tabular}

${ }^{a}$ All calculations performed based on SA-CASSCF/cc-pVTZ wave functions computed with equal weights for the $\mathrm{S}_{0}, \mathrm{~S}_{1}$ and $\mathrm{S}_{2}$ states. ${ }^{b}$ Only MEP points in the $\mathrm{S}_{2}$ state are shown. ${ }^{c}$ Dihedral angles defined in Scheme 1. 


\begin{tabular}{|c|c|c|c|c|}
\hline \multicolumn{5}{|c|}{ Table S6. Vertical CASPT2/cc-pVTZ Excitation Energies (eV) of the $E$ and $Z$ Isomers of Motor 2} \\
\hline \multirow[b]{2}{*}{ isomer } & \multirow[b]{2}{*}{ excitation } & \multicolumn{3}{|c|}{$\mathrm{S}_{0}$ geometry } \\
\hline & & $\begin{array}{l}\text { CASSCF/ } \\
\text { cc-pVTZ }\end{array}$ & $\begin{array}{l}\text { RI-MP2/ } \\
\text { cc-pVDZ }\end{array}$ & $\begin{array}{l}\text { RI-MP2/ } \\
\text { def2-SVP }\end{array}$ \\
\hline \multirow[t]{2}{*}{$E$} & $\mathrm{~S}_{0} \rightarrow \mathrm{S}_{1}$ & $4.25(0.293)$ & $4.12(0.243)$ & $4.06(0.227)$ \\
\hline & $\mathrm{S}_{0} \rightarrow \mathrm{S}_{2}$ & $5.43(0.050)$ & $5.36(0.032)$ & $5.32(0.031)$ \\
\hline \multirow[t]{2}{*}{$Z$} & $\mathrm{~S}_{0} \rightarrow \mathrm{S}_{1}$ & $4.09(0.298)$ & $3.94(0.239)$ & $3.88(0.226)$ \\
\hline & $\mathrm{S}_{0} \rightarrow \mathrm{S}_{2}$ & $5.46(0.031)$ & $5.32(0.025)$ & $5.28(0.024)$ \\
\hline
\end{tabular}

${ }^{a}$ All calculations performed using state-specific CASPT2, based on SA-CASSCF wave functions computed with equal weights for the $\mathrm{S}_{0}, \mathrm{~S}_{1}$ and $\mathrm{S}_{2}$ states. Oscillator strengths in parentheses obtained with the complete active space state-interaction (CASSI) method (Ref. 20). 


\begin{tabular}{|c|c|c|}
\hline isomer & $\mathrm{S}_{2} \mathrm{FC}$ point & $\mathrm{S}_{2}$ MEP point 5 \\
\hline$E$ & -31 & -82 \\
\hline$Z$ & -35 & -82 \\
\hline
\end{tabular}

Comment on Table S7: As a clear sign that the cyclopentadiene motif gains aromaticity during the photoisomerizations, the NICS $(1)_{z z}$ values are much more negative at the fifth $\mathrm{S}_{2}$ MEP points than at the $\mathrm{S}_{2} \mathrm{FC}$ points. 


\section{Description of multimedia files}

1E-Z-E.avi This movie from the NAMD simulations of motor 1 shows two representative trajectories merged together to illustrate a full $360^{\circ} E \rightarrow Z \rightarrow E$ rotation around the central olefinic bond.

2E-Z-E.avi This movie from the NAMD simulations of motor $\mathbf{2}$ shows two representative trajectories merged together to illustrate a full $360^{\circ} E \rightarrow Z \rightarrow E$ rotation around the central olefinic bond. 


\section{References for the Supporting Information}

(1) Roos, B. O.; Taylor, P. R.; Siegbahn, P. E. M. Chem. Phys. 1980, 48, 157-173.

(2) Dunning, T. H., Jr. J. Chem. Phys. 1989, 90, 1007-1023.

(3) (a) Andersson, K.; Malmqvist, P.-Å.; Roos, B. O. J. Chem. Phys. 1992, 96, 1218-1226. (b) Finley, J.; Malmqvist, P.-Å.; Roos, B. O.; Serrano-Andrés, L. Chem. Phys. Lett. 1998, 288, 299-306.

(4) (a) Hariharan, P. C.; Pople, J. A. Theor. Chim. Acta 1973, 28, 213-222. (b) Francl, M. M.; Pietro, W. J.; Hehre, W. J.; Binkley, J. S.; Gordon, M. S.; DeFrees, D. J.; Pople, J. A. J. Chem. Phys. 1982, 77, 3654-3665.

(5) (a) Weigend, F.; Ahlrichs, R. Phys. Chem. Chem. Phys. 2005, 7, 3297-3305. (b) Weigend, F. Phys. Chem. Chem. Phys. 2006, 8, 1057-1065.

(6) (a) Feyereisen, M.; Fitzgerald, G.; Komornicki, A. Chem. Phys. Lett. 1993, 208, 359-363.

(b) Vahtras, O.; Almlöf, J.; Feyereisen, M. W. Chem. Phys. Lett. 1993, 213, 514-518.

(a) Dahl, J. P.; Springborg, M. J. Chem. Phys. 1988, 88, 4535-4547. (b) Schinke, R. Photodissociation Dynamics: Spectroscopy and Fragmentation of Small Polyatomic Molecules, Cambridge University Press, Cambridge, U.K., 1993.

(8) Verlet, L. Phys. Rev. 1967, 159, 98-103.

(9) (a) Groenhof, G.; Bouxin-Cademartory, M.; Hess, B.; de Visser, S. P.; Berendsen, H. J. C.; Olivucci, M.; Mark, A. E.; Robb, M. A. J. Am. Chem. Soc. 2004, 126, 4228-4233. (b) Schäfer, L. V.; Groenhof, G.; Boggio-Pasqua, M.; Robb, M. A.; Grubmüller, H. PLoS Comput. Biol. 2008, 4, e1000034.

(10) Aquilante, F.; Autschbach, J.; Carlson, R. K.; Chibotaru, L. F.; Delcey, M. G.; De Vico, L.; Fdez. Galván, I.; Ferré, N.; Frutos, L. M.; Gagliardi, L.; Garavelli, M.; Giussani, A.; Hoyer, C. E.; Manni, G. L.; Lischka, H.; Ma, D.; Malmqvist, P.-Å.; Müller, T.; Nenov, A.; Olivucci, M.; Pedersen, T. B.; Peng, D.; Plasser, F.; Pritchard, B.; Reiher, M.; Rivalta, I.; Schapiro, I.; Segarra-Martí, J.; Stenrup, M.; Truhlar, D. G.; Ungur, L.; Valentini, A.; Vancoillie, S.; Veryazov, V.; Vysotskiy, V. P.; Weingart, O.; Zapata, F.; Lindh, R. J. Comput. Chem. 2016, 37, 506-541.

(11) (a) Desouter-Lecomte, M.; Lorquet, J. C. J. Chem. Phys. 1979, 71, 4391-4403. (b) Wittig, C. J. Phys. Chem. B 2005, 109, 8428-8430. 
(12) (a) Noorizadeh, S.; Shakerzadeh, E. Phys. Chem. Chem. Phys. 2010, 12, 4742-4749. (b) Noorizadeh, S.; Shakerzadeh, E. Comput. Theo. Chem. 2011, 964, 141-147.

(13) (a) Kruszewski, J.; Krygowki, T. M. Tetrahedron Lett. 1972, 13, 3839-3842. (b) Krygowki, T. M. J. Chem. Inf. Comput. Sci. 1993, 33, 70-78. (c) Krygowski, T. M.; Cyrański, M. K. Chem. Rev. 2001, 101, 1385-1419.

(14) Bader, R. F. W. Chem. Rev. 1991, 91, 893-928.

(15) Shannon, C. E. Bell Syst. Tech. J. 1948, 27, 379-423.

(16) (a) von Ragué Schleyer, P.; Maerker, C.; Dransfeld, A.; Jiao, H.; van Eikema Hommes, N. J. R. J. Am. Chem. Soc. 1996, 118, 6317-6318. (b) Karadakov, P. B. J. Phys. Chem. A 2008, 112, 7303-7309. (c) Karadakov, P. B. J. Phys. Chem. A 2008, 112, 12707-12713.

(17) (a) Ahlrichs, R.; Bär, M.; Häser, M.; Horn, H.; Kölmel, C. Chem. Phys. Lett. 1989, 162, 165-169. (b) TURBOMOLE V6.3 2011, a development of University of Karlsruhe and Forschungszentrum Karlsruhe GmbH, 1989-2007, TURBOMOLE GmbH, since 2007; available at: http://www.turbomole.com (accessed July 18, 2017).

(18) Lu, T.; Chen, F. J. Comput. Chem. 2012, 33, 580-592.

(19) (a) Aidas, K.; Angeli, C.; Bak, K. L.; Bakken, V.; Bast, R.; Boman, L.; Christiansen, O.; Cimiraglia, R.; Coriani, S.; Dahle, P.; Dalskov, E. K.; Ekström, U.; Enevoldsen, T.; Eriksen, J. J.; Ettenhuber, P.; Fernández, B.; Ferrighi, L.; Fliegl, H.; Frediani, L.; Hald, K.; Halkier, A.; Hättig, C.; Heiberg, H.; Helgaker, T.; Hennum, A. C.; Hettema, H.; Hjertenæs, E.; Høst, S.; Høyvik, I.-M.; Iozzi, M. F.; Jansík, B.; Aa. Jensen, H. J.; Jonsson, D.; Jørgensen, P.; Kauczor, J.; Kirpekar, S.; Kjærgaard, T.; Klopper, W.; Knecht, S.; Kobayashi, R.; Koch, H.; Kongsted, J.; Krapp, A.; Kristensen, K.; Ligabue, A.; Lutnæs, O. B.; Melo, J. I.; Mikkelsen, K. V.; Myhre, R. H.; Neiss, C.; Nielsen C. B.; Norman, P.; Olsen, J.; Olsen, J. M. H.; Osted, A.; Packer, M. J.; Pawlowski, F.; Pedersen, T. B.; Provasi, P. F.; Reine, S.; Rinkevicius, Z.; Ruden, T. A.; Ruud, K.; Rybkin, V. V.; Salek, P.; Samson, C. C. M.; Sánchez de Merás, A.; Saue, T.; Sauer, S. P. A.; Schimmelpfennig, B.; Sneskov, K.; Steindal, A. H.; SylvesterHvid, K. O.; Taylor, P. R.; Teale, A. M.; Tellgren, E. I.; Tew, D. P.; Thorvaldsen, A. J.; Thøgersen, L.; Vahtras, O.; Watson, M. A.; Wilson, D. J. D.; Ziolkowski, M.; Ågren, H. WIREs Comput. Mol. Sci. 2014, 4, 269-284. (b) Dalton, a molecular electronic structure program, release Dalton2013.4 (2014), see http://daltonprogram.org.

(20) Malmqvist, P.-Å.; Roos, B. O. Chem. Phys. Lett. 1989, 155, 189-194. 


\section{Cartesian coordinates ( $(\AA)$ and energies (a.u.) of different geometries of motors 1 and 2}

1-E, $\mathrm{S}_{0}$ geometry

CASSCF/cc-pVTZ S $\mathrm{S}_{0}$ energy: -518.96617911

$\begin{array}{lrrc}\text { C } & -0.70961096 & 0.04538929 & -0.14267191 \\ \text { C } & 0.62712810 & 0.28221913 & -0.08052262 \\ \text { C } & 1.38252611 & 1.53489753 & 0.05711937 \\ \text { C } & 1.65428313 & -0.76385311 & -0.17239699 \\ \text { C } & 2.88325190 & -0.20319303 & -0.09920924 \\ \text { C } & 2.70352420 & 1.24501346 & 0.04611591 \\ \text { N } & -1.74542353 & 0.94454595 & -0.10787502 \\ \text { C } & -3.04396237 & 0.30758001 & -0.15944937 \\ \text { H } & 3.50304009 & 1.95501601 & 0.12754225 \\ \text { C } & -1.31683303 & -1.34602708 & -0.26193028 \\ \text { C } & -2.73238851 & -1.05018967 & -0.77166409 \\ \text { H } & -2.72833176 & -0.96572500 & -1.85147653 \\ \text { H } & -3.44956650 & -1.81146443 & -0.49552296 \\ \text { H } & -3.48449265 & 0.21156146 & 0.83243180 \\ \text { H } & -3.73009490 & 0.88933001 & -0.76272180 \\ \text { H } & -0.76757444 & -1.94211545 & -0.97539308 \\ \text { H } & 0.97319171 & 2.51528572 & 0.15074512 \\ \text { C } & -1.64278234 & 2.31511402 & 0.31127943 \\ \text { H } & -1.13636746 & 2.41072704 & 1.26659410 \\ \text { H } & -1.11701016 & 2.91969401 & -0.41742632 \\ \text { H } & -2.63895578 & 2.72105466 & 0.41729056 \\ \text { C } & -1.33279089 & -2.07540993 & 1.08591288 \\ \text { H } & -1.79995785 & -3.04855087 & 0.98020735 \\ \text { H } & -0.33120259 & -2.21824514 & 1.46688796 \\ \text { H } & -1.88995641 & -1.51699265 & 1.83012818 \\ \text { H } & 1.45983655 & -1.80976523 & -0.29044185 \\ \text { C } & 4.21266196 & -0.89009220 & -0.15727088 \\ \text { H } & 4.80471128 & -0.52919550 & -0.99255379 \\ \text { H } & 4.78790745 & -0.70880124 & 0.74535744 \\ \text { H } & 4.09605364 & -1.96076373 & -0.26898660\end{array}$


1-E, MEP point $1\left(\mathrm{~S}_{2}\right)$

SA-CASSCF/cc-pVTZ S $S_{0}$ energy: -518.93470749

SA-CASSCF/cc-pVTZ S S $_{1}$ energy: -518.83174355

SA-CASSCF/cc-pVTZ S S $_{2}$ energy: -518.78130766

$\begin{array}{lrrc}\mathrm{C} & -0.777366 & 0.021368 & -0.044232 \\ \mathrm{C} & 0.690352 & 0.295863 & -0.048030 \\ \mathrm{C} & 1.362454 & 1.528289 & 0.079874 \\ \mathrm{C} & 1.640057 & -0.740818 & -0.198296 \\ \mathrm{C} & 2.933890 & -0.166793 & -0.128563 \\ \mathrm{C} & 2.760247 & 1.251023 & 0.037387 \\ \mathrm{~N} & -1.741537 & 0.944018 & -0.112075 \\ \mathrm{C} & -3.060338 & 0.333530 & -0.211196 \\ \mathrm{H} & 3.546388 & 1.970680 & 0.140151 \\ \mathrm{C} & -1.348466 & -1.349777 & -0.233149 \\ \mathrm{C} & -2.762381 & -1.055579 & -0.758294 \\ \mathrm{H} & -2.756034 & -1.022975 & -1.840273 \\ \mathrm{H} & -3.494492 & -1.787731 & -0.448225 \\ \mathrm{H} & -3.520568 & 0.299619 & 0.773812 \\ \mathrm{H} & -3.698037 & 0.924722 & -0.853735 \\ \mathrm{H} & -0.769673 & -1.896676 & -0.967152 \\ \mathrm{H} & 0.943766 & 2.498234 & 0.214283 \\ \mathrm{C} & -1.647896 & 2.336039 & 0.272715 \\ \mathrm{H} & -1.174332 & 2.441699 & 1.239553 \\ \mathrm{H} & -1.091558 & 2.905462 & -0.455540 \\ \mathrm{H} & -2.648756 & 2.736326 & 0.330250 \\ \mathrm{C} & -1.343852 & -2.143289 & 1.081736 \\ \mathrm{H} & -1.731274 & -3.142933 & 0.917697 \\ \mathrm{H} & -0.342677 & -2.226112 & 1.480078 \\ \mathrm{H} & -1.963001 & -1.660907 & 1.830239 \\ \mathrm{H} & 1.434945 & -1.783422 & -0.319186 \\ \mathrm{C} & 4.238716 & -0.897036 & -0.168023 \\ \mathrm{H} & 4.874662 & -0.536498 & -0.971836 \\ \mathrm{H} & 4.796983 & -0.777779 & 0.756746 \\ \mathrm{H} & 4.086011 & -1.958714 & -0.322785\end{array}$


1-E, MEP point $2\left(\mathrm{~S}_{2}\right)$

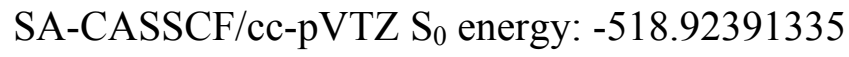

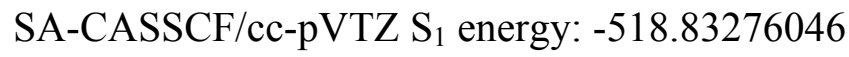

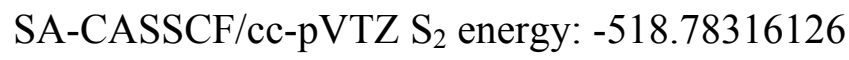

$\begin{array}{lrrc}\mathrm{C} & -0.795225 & 0.019437 & 0.066775 \\ \mathrm{C} & 0.695442 & 0.298779 & 0.003654 \\ \mathrm{C} & 1.375166 & 1.520839 & 0.176120 \\ \mathrm{C} & 1.640270 & -0.719296 & -0.261778 \\ \mathrm{C} & 2.931179 & -0.152499 & -0.186617 \\ \mathrm{C} & 2.761602 & 1.252040 & 0.061699 \\ \mathrm{~N} & -1.730091 & 0.947409 & -0.143250 \\ \mathrm{C} & -3.055795 & 0.356870 & -0.279808 \\ \mathrm{H} & 3.550922 & 1.965457 & 0.186340 \\ \mathrm{C} & -1.356826 & -1.352243 & -0.175545 \\ \mathrm{C} & -2.763828 & -1.065982 & -0.734080 \\ \mathrm{H} & -2.748138 & -1.103802 & -1.815355 \\ \mathrm{H} & -3.508240 & -1.768396 & -0.386974 \\ \mathrm{H} & -3.563339 & 0.389750 & 0.681643 \\ \mathrm{H} & -3.646028 & 0.925295 & -0.984822 \\ \mathrm{H} & -0.754071 & -1.855864 & -0.921785 \\ \mathrm{H} & 0.961647 & 2.479539 & 0.389916 \\ \mathrm{C} & -1.640280 & 2.354067 & 0.196185 \\ \mathrm{H} & -1.246996 & 2.484986 & 1.195714 \\ \mathrm{H} & -1.013029 & 2.883054 & -0.502577 \\ \mathrm{H} & -2.634231 & 2.773002 & 0.156074 \\ \mathrm{C} & -1.355016 & -2.204620 & 1.097860 \\ \mathrm{H} & -1.701613 & -3.208054 & 0.877105 \\ \mathrm{H} & -0.359366 & -2.272497 & 1.514053 \\ \mathrm{H} & -2.007567 & -1.780790 & 1.853273 \\ \mathrm{H} & 1.432878 & -1.757041 & -0.419564 \\ \mathrm{C} & 4.234360 & -0.890982 & -0.216300 \\ \mathrm{H} & 4.919426 & -0.463536 & -0.942893 \\ \mathrm{H} & 4.736421 & -0.866008 & 0.747457 \\ \mathrm{H} & 4.085714 & -1.931962 & -0.479011\end{array}$


1-E, MEP point $3\left(\mathrm{~S}_{2}\right)$

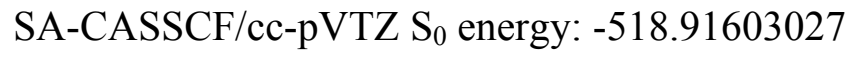

SA-CASSCF/cc-pVTZ S S $_{1}$ energy: -518.83283303

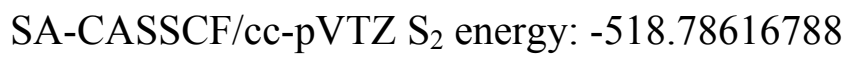

$\begin{array}{lrrc}\mathrm{C} & -0.804556 & 0.022089 & 0.120064 \\ \mathrm{C} & 0.693942 & 0.298333 & 0.048355 \\ \mathrm{C} & 1.383303 & 1.497898 & 0.312726 \\ \mathrm{C} & 1.627507 & -0.690194 & -0.343997 \\ \mathrm{C} & 2.917739 & -0.135441 & -0.241575 \\ \mathrm{C} & 2.758126 & 1.242420 & 0.127186 \\ \mathrm{~N} & -1.715120 & 0.945390 & -0.184378 \\ \mathrm{C} & -3.043821 & 0.365431 & -0.339041 \\ \mathrm{H} & 3.553443 & 1.942124 & 0.287870 \\ \mathrm{C} & -1.357995 & -1.354979 & -0.120498 \\ \mathrm{C} & -2.758309 & -1.083278 & -0.706725 \\ \mathrm{H} & -2.735348 & -1.185665 & -1.783461 \\ \mathrm{H} & -3.512333 & -1.757264 & -0.325815 \\ \mathrm{H} & -3.583302 & 0.457449 & 0.600906 \\ \mathrm{H} & -3.600294 & 0.903594 & -1.093301 \\ \mathrm{H} & -0.742318 & -1.846046 & -0.864259 \\ \mathrm{H} & 0.975205 & 2.433134 & 0.623869 \\ \mathrm{C} & -1.623848 & 2.363205 & 0.110424 \\ \mathrm{H} & -1.342306 & 2.521445 & 1.144177 \\ \mathrm{H} & -0.906191 & 2.847297 & -0.529468 \\ \mathrm{H} & -2.594681 & 2.804074 & -0.056342 \\ \mathrm{C} & -1.358359 & -2.221880 & 1.139910 \\ \mathrm{H} & -1.697097 & -3.223963 & 0.902547 \\ \mathrm{H} & -0.363683 & -2.290430 & 1.559157 \\ \mathrm{H} & -2.017450 & -1.813385 & 1.897915 \\ \mathrm{H} & 1.416046 & -1.714187 & -0.571390 \\ \mathrm{C} & 4.217108 & -0.880738 & -0.288885 \\ \mathrm{H} & 4.938643 & -0.384107 & -0.931047 \\ \mathrm{H} & 4.670799 & -0.963028 & 0.695613 \\ \mathrm{H} & 4.077912 & -1.886727 & -0.667788\end{array}$


1-E, MEP point $4\left(\mathrm{~S}_{2}\right)$

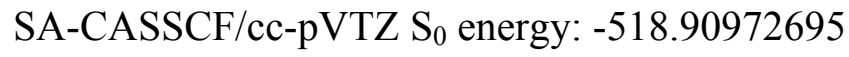

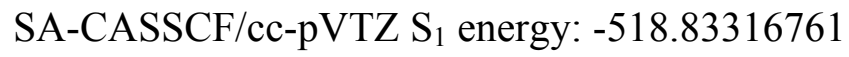

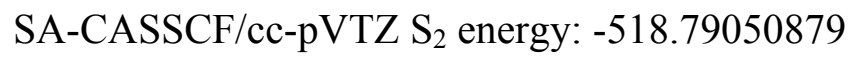

$\begin{array}{lrrc}\mathrm{C} & -0.809663 & 0.024322 & 0.141087 \\ \mathrm{C} & 0.691917 & 0.295076 & 0.078945 \\ \mathrm{C} & 1.391447 & 1.459013 & 0.453177 \\ \mathrm{C} & 1.616579 & -0.648906 & -0.431386 \\ \mathrm{C} & 2.907674 & -0.112498 & -0.285680 \\ \mathrm{C} & 2.757445 & 1.225691 & 0.212022 \\ \mathrm{~N} & -1.701662 & 0.939464 & -0.230256 \\ \mathrm{C} & -3.034158 & 0.368364 & -0.386676 \\ \mathrm{H} & 3.557503 & 1.907474 & 0.420087 \\ \mathrm{C} & -1.363350 & -1.358560 & -0.073039 \\ \mathrm{C} & -2.760998 & -1.100975 & -0.675050 \\ \mathrm{H} & -2.739295 & -1.263226 & -1.744272 \\ \mathrm{H} & -3.522237 & -1.745243 & -0.258711 \\ \mathrm{H} & -3.592421 & 0.514514 & 0.535056 \\ \mathrm{H} & -3.567137 & 0.874474 & -1.179038 \\ \mathrm{H} & -0.744262 & -1.854698 & -0.810240 \\ \mathrm{H} & 0.984208 & 2.359577 & 0.858205 \\ \mathrm{C} & -1.604843 & 2.364863 & 0.027239 \\ \mathrm{H} & -1.452700 & 2.550050 & 1.084831 \\ \mathrm{H} & -0.796024 & 2.805222 & -0.528387 \\ \mathrm{H} & -2.532548 & 2.825833 & -0.275900 \\ \mathrm{C} & -1.359796 & -2.217505 & 1.190610 \\ \mathrm{H} & -1.694200 & -3.221926 & 0.957401 \\ \mathrm{H} & -0.363636 & -2.280944 & 1.607679 \\ \mathrm{H} & -2.019541 & -1.809155 & 1.947953 \\ \mathrm{H} & 1.399720 & -1.649165 & -0.744057 \\ \mathrm{C} & 4.201566 & -0.866651 & -0.365153 \\ \mathrm{H} & 4.946349 & -0.321974 & -0.937955 \\ \mathrm{H} & 4.624422 & -1.043354 & 0.620676 \\ \mathrm{H} & 4.064483 & -1.832345 & -0.838160\end{array}$


1-E, MEP point $5\left(\mathrm{~S}_{2}\right)$

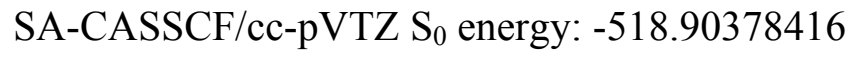

SA-CASSCF/cc-pVTZ $S_{1}$ energy: -518.83350211

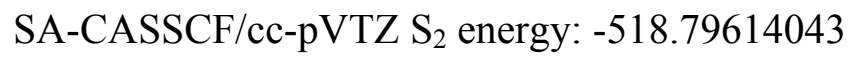

$\begin{array}{lrrc}\mathrm{C} & -0.810946 & 0.026463 & 0.147796 \\ \mathrm{C} & 0.688510 & 0.289705 & 0.101205 \\ \mathrm{C} & 1.397807 & 1.404877 & 0.589312 \\ \mathrm{C} & 1.605793 & -0.597048 & -0.519096 \\ \mathrm{C} & 2.897257 & -0.085489 & -0.322451 \\ \mathrm{C} & 2.755711 & 1.199507 & 0.302959 \\ \mathrm{~N} & -1.687106 & 0.931429 & -0.278578 \\ \mathrm{C} & -3.023912 & 0.368785 & -0.428162 \\ \mathrm{H} & 3.559784 & 1.859467 & 0.560406 \\ \mathrm{C} & -1.369273 & -1.360973 & -0.028482 \\ \mathrm{C} & -2.765722 & -1.116530 & -0.640981 \\ \mathrm{H} & -2.749111 & -1.335233 & -1.700101 \\ \mathrm{H} & -3.532895 & -1.729757 & -0.190058 \\ \mathrm{H} & -3.593759 & 0.566126 & 0.476781 \\ \mathrm{H} & -3.539535 & 0.841789 & -1.251801 \\ \mathrm{H} & -0.749908 & -1.869596 & -0.756961 \\ \mathrm{H} & 0.989105 & 2.263058 & 1.079589 \\ \mathrm{C} & -1.583102 & 2.361800 & -0.052982 \\ \mathrm{H} & -1.556711 & 2.574122 & 1.011227 \\ \mathrm{H} & -0.699669 & 2.762984 & -0.515215 \\ \mathrm{H} & -2.453289 & 2.836549 & -0.480288 \\ \mathrm{C} & -1.361657 & -2.203078 & 1.244563 \\ \mathrm{H} & -1.695384 & -3.210296 & 1.023424 \\ \mathrm{H} & -0.363603 & -2.261219 & 1.658398 \\ \mathrm{H} & -2.019335 & -1.787178 & 1.999549 \\ \mathrm{H} & 1.382826 & -1.564813 & -0.918651 \\ \mathrm{C} & 4.185675 & -0.845702 & -0.440482 \\ \mathrm{H} & 4.947570 & -0.264767 & -0.951975 \\ \mathrm{H} & 4.585693 & -1.106119 & 0.536068 \\ \mathrm{H} & 4.049141 & -1.768414 & -0.992810\end{array}$


1-E, MEP point $6\left(\mathrm{~S}_{2}\right)$

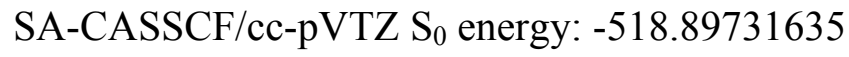

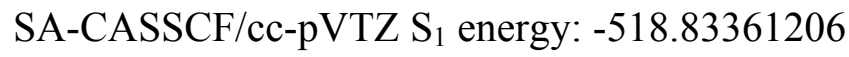

SA-CASSCF/cc-pVTZ S S $_{2}$ energy: -518.80290761

$\begin{array}{lrrc}\mathrm{C} & -0.809277 & 0.028289 & 0.145710 \\ \mathrm{C} & 0.683709 & 0.284159 & 0.117666 \\ \mathrm{C} & 1.403244 & 1.336861 & 0.718922 \\ \mathrm{C} & 1.595409 & -0.534321 & -0.602713 \\ \mathrm{C} & 2.887205 & -0.055392 & -0.353095 \\ \mathrm{C} & 2.753870 & 1.163864 & 0.395276 \\ \mathrm{~N} & -1.672300 & 0.921415 & -0.327721 \\ \mathrm{C} & -3.013542 & 0.367368 & -0.465965 \\ \mathrm{H} & 3.561559 & 1.797803 & 0.702238 \\ \mathrm{C} & -1.375911 & -1.362369 & 0.014354 \\ \mathrm{C} & -2.771568 & -1.130063 & -0.606148 \\ \mathrm{H} & -2.761810 & -1.402032 & -1.652921 \\ \mathrm{H} & -3.544277 & -1.710907 & -0.122918 \\ \mathrm{H} & -3.590586 & 0.613040 & 0.422509 \\ \mathrm{H} & -3.515974 & 0.806588 & -1.316075 \\ \mathrm{H} & -0.757565 & -1.887493 & -0.703473 \\ \mathrm{H} & 0.992448 & 2.147891 & 1.284277 \\ \mathrm{C} & -1.560065 & 2.355369 & -0.130519 \\ \mathrm{H} & -1.650220 & 2.595178 & 0.925400 \\ \mathrm{H} & -0.619404 & 2.722302 & -0.497207 \\ \mathrm{H} & -2.363638 & 2.838455 & -0.665892 \\ \mathrm{C} & -1.364401 & -2.181892 & 1.300517 \\ \mathrm{H} & -1.699266 & -3.192242 & 1.096282 \\ \mathrm{H} & -0.364370 & -2.234522 & 1.710486 \\ \mathrm{H} & -2.018776 & -1.754052 & 2.051520 \\ \mathrm{H} & 1.364697 & -1.460191 & -1.088950 \\ \mathrm{C} & 4.170050 & -0.818833 & -0.514704 \\ \mathrm{H} & 4.945147 & -0.209729 & -0.970458 \\ \mathrm{H} & 4.552959 & -1.156120 & 0.445047 \\ \mathrm{H} & 4.031686 & -1.695810 & -1.136530\end{array}$


1-E, MEP point $7\left(\mathrm{~S}_{2}\right)$

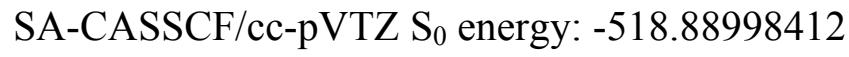

SA-CASSCF/cc-pVTZ S S $_{1}$ energy: -518.83338678

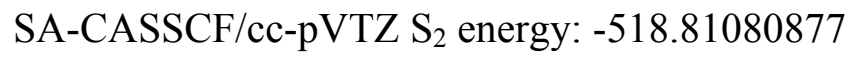

$\begin{array}{lrrc}\mathrm{C} & -0.805016 & 0.029755 & 0.138361 \\ \mathrm{C} & 0.677686 & 0.279703 & 0.129643 \\ \mathrm{C} & 1.407940 & 1.257029 & 0.839083 \\ \mathrm{C} & 1.585632 & -0.461564 & -0.679792 \\ \mathrm{C} & 2.877288 & -0.023390 & -0.377474 \\ \mathrm{C} & 2.751737 & 1.117538 & 0.487286 \\ \mathrm{~N} & -1.657473 & 0.909685 & -0.376040 \\ \mathrm{C} & -3.002951 & 0.364116 & -0.501781 \\ \mathrm{H} & 3.563074 & 1.720026 & 0.843947 \\ \mathrm{C} & -1.382459 & -1.362271 & 0.056039 \\ \mathrm{C} & -2.777869 & -1.141519 & -0.570593 \\ \mathrm{H} & -2.776638 & -1.465164 & -1.602540 \\ \mathrm{H} & -3.555334 & -1.688410 & -0.056380 \\ \mathrm{H} & -3.585532 & 0.656298 & 0.368800 \\ \mathrm{H} & -3.493752 & 0.768733 & -1.375597 \\ \mathrm{H} & -0.766050 & -1.905638 & -0.650180 \\ \mathrm{H} & 0.996447 & 2.012824 & 1.476878 \\ \mathrm{C} & -1.536933 & 2.346232 & -0.206453 \\ \mathrm{H} & -1.731541 & 2.614637 & 0.829172 \\ \mathrm{H} & -0.555034 & 2.684093 & -0.480875 \\ \mathrm{H} & -2.269694 & 2.832020 & -0.833877 \\ \mathrm{C} & -1.367813 & -2.155199 & 1.357296 \\ \mathrm{H} & -1.705550 & -3.168457 & 1.173354 \\ \mathrm{H} & -0.366060 & -2.202573 & 1.763820 \\ \mathrm{H} & -2.018218 & -1.711970 & 2.102733 \\ \mathrm{H} & 1.346264 & -1.337264 & -1.248652 \\ \mathrm{C} & 4.154420 & -0.785994 & -0.588312 \\ \mathrm{H} & 4.940364 & -0.153445 & -0.990371 \\ \mathrm{H} & 4.524119 & -1.197659 & 0.347140 \\ \mathrm{H} & 4.012866 & -1.612598 & -1.274716\end{array}$


1-E, MEP point $8\left(\mathrm{~S}_{2}\right)$

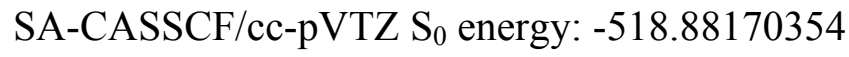

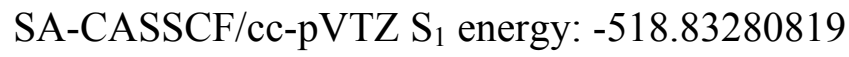

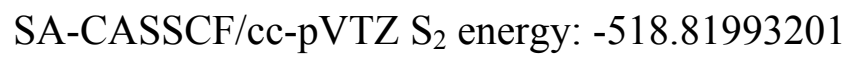

$\begin{array}{lrrc}\mathrm{C} & -0.798506 & 0.030645 & 0.124905 \\ \mathrm{C} & 0.670501 & 0.277446 & 0.136799 \\ \mathrm{C} & 1.411769 & 1.165409 & 0.949828 \\ \mathrm{C} & 1.577088 & -0.379719 & -0.749218 \\ \mathrm{C} & 2.868480 & 0.009096 & -0.395541 \\ \mathrm{C} & 2.749733 & 1.060108 & 0.577804 \\ \mathrm{~N} & -1.643767 & 0.896667 & -0.423939 \\ \mathrm{C} & -2.993196 & 0.358905 & -0.533975 \\ \mathrm{H} & 3.563710 & 1.627247 & 0.983424 \\ \mathrm{C} & -1.389025 & -1.360323 & 0.097253 \\ \mathrm{C} & -2.783645 & -1.151263 & -0.535812 \\ \mathrm{H} & -2.789153 & -1.521822 & -1.551864 \\ \mathrm{H} & -3.565691 & -1.665659 & 0.004581 \\ \mathrm{H} & -3.577888 & 0.693785 & 0.319641 \\ \mathrm{H} & -3.476466 & 0.729142 & -1.427209 \\ \mathrm{H} & -0.775337 & -1.925285 & -0.594525 \\ \mathrm{H} & 1.000745 & 1.863239 & 1.651999 \\ \mathrm{C} & -1.514756 & 2.335427 & -0.280863 \\ \mathrm{H} & -1.794627 & 2.630900 & 0.727657 \\ \mathrm{H} & -0.506119 & 2.648597 & -0.475445 \\ \mathrm{H} & -2.180506 & 2.818773 & -0.980923 \\ \mathrm{C} & -1.372404 & -2.122692 & 1.415551 \\ \mathrm{H} & -1.713797 & -3.138454 & 1.254044 \\ \mathrm{H} & -0.369005 & -2.164981 & 1.818775 \\ \mathrm{H} & -2.018540 & -1.662162 & 2.154215 \\ \mathrm{H} & 1.329403 & -1.196621 & -1.397919 \\ \mathrm{C} & 4.140045 & -0.747329 & -0.660751 \\ \mathrm{H} & 4.933981 & -0.094925 & -1.012130 \\ \mathrm{H} & 4.500546 & -1.229484 & 0.243787 \\ \mathrm{H} & 3.993556 & -1.520197 & -1.406243\end{array}$


1-E, MEP point $9\left(\mathrm{~S}_{2}\right)$

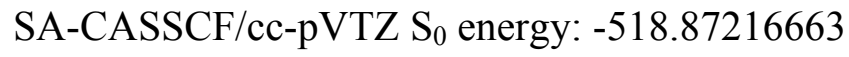

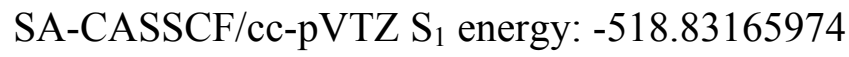

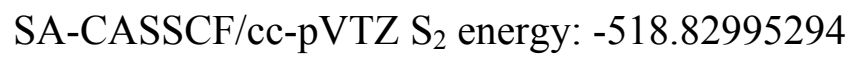

$\begin{array}{lrrc}\mathrm{C} & -0.790356 & 0.030646 & 0.108313 \\ \mathrm{C} & 0.662875 & 0.276234 & 0.141887 \\ \mathrm{C} & 1.414898 & 1.064305 & 1.049177 \\ \mathrm{C} & 1.569513 & -0.291000 & -0.808153 \\ \mathrm{C} & 2.860507 & 0.042472 & -0.408401 \\ \mathrm{C} & 2.747433 & 0.992058 & 0.664442 \\ \mathrm{~N} & -1.630927 & 0.882590 & -0.469589 \\ \mathrm{C} & -2.983764 & 0.352234 & -0.564464 \\ \mathrm{H} & 3.564272 & 1.516326 & 1.119273 \\ \mathrm{C} & -1.395476 & -1.356655 & 0.137150 \\ \mathrm{C} & -2.789742 & -1.159200 & -0.500567 \\ \mathrm{H} & -2.803580 & -1.575187 & -1.498791 \\ \mathrm{H} & -3.575517 & -1.640064 & 0.064853 \\ \mathrm{H} & -3.570419 & 0.728030 & 0.270522 \\ \mathrm{H} & -3.460020 & 0.687656 & -1.475255 \\ \mathrm{H} & -0.785554 & -1.943593 & -0.539656 \\ \mathrm{H} & 1.005537 & 1.695853 & 1.812852 \\ \mathrm{C} & -1.493286 & 2.323074 & -0.355221 \\ \mathrm{H} & -1.842677 & 2.646706 & 0.622798 \\ \mathrm{H} & -0.467926 & 2.616462 & -0.481498 \\ \mathrm{H} & -2.097712 & 2.799520 & -1.113714 \\ \mathrm{C} & -1.377170 & -2.085389 & 1.473370 \\ \mathrm{H} & -1.724058 & -3.102923 & 1.336866 \\ \mathrm{H} & -0.372232 & -2.123366 & 1.873200 \\ \mathrm{H} & -2.018214 & -1.604936 & 2.203778 \\ \mathrm{H} & 1.314399 & -1.040724 & -1.531719 \\ \mathrm{C} & 4.126304 & -0.702132 & -0.731681 \\ \mathrm{H} & 4.927334 & -0.032851 & -1.031258 \\ \mathrm{H} & 4.479496 & -1.254496 & 0.134588 \\ \mathrm{H} & 3.974100 & -1.414976 & -1.533576\end{array}$


1-E, MEP point $10\left(\mathrm{~S}_{1}\right)$

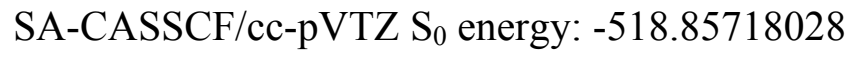

SA-CASSCF/cc-pVTZ S S $_{1}$ energy: -518.85672015

SA-CASSCF/cc-pVTZ S S $_{2}$ energy: -518.81258442

$\begin{array}{lrrc}\mathrm{C} & -0.790905 & 0.022121 & 0.151007 \\ \mathrm{C} & 0.648875 & 0.275565 & 0.145686 \\ \mathrm{C} & 1.376357 & 1.061932 & 1.024541 \\ \mathrm{C} & 1.554263 & -0.304343 & -0.780507 \\ \mathrm{C} & 2.899080 & 0.067490 & -0.377880 \\ \mathrm{C} & 2.786894 & 0.967542 & 0.640362 \\ \mathrm{~N} & -1.647510 & 0.901097 & -0.505428 \\ \mathrm{C} & -2.981148 & 0.360139 & -0.556996 \\ \mathrm{H} & 3.590063 & 1.453728 & 1.154506 \\ \mathrm{C} & -1.385618 & -1.362536 & 0.123837 \\ \mathrm{C} & -2.786962 & -1.157730 & -0.499508 \\ \mathrm{H} & -2.809618 & -1.574261 & -1.498207 \\ \mathrm{H} & -3.570745 & -1.641641 & 0.069072 \\ \mathrm{H} & -3.587997 & 0.707558 & 0.283977 \\ \mathrm{H} & -3.480759 & 0.681123 & -1.464885 \\ \mathrm{H} & -0.801165 & -1.987114 & -0.550709 \\ \mathrm{H} & 0.987953 & 1.658718 & 1.822497 \\ \mathrm{C} & -1.501987 & 2.316714 & -0.352909 \\ \mathrm{H} & -1.833676 & 2.675743 & 0.626822 \\ \mathrm{H} & -0.472305 & 2.614462 & -0.489134 \\ \mathrm{H} & -2.094799 & 2.818552 & -1.108904 \\ \mathrm{C} & -1.379018 & -2.082374 & 1.473373 \\ \mathrm{H} & -1.730017 & -3.102838 & 1.353922 \\ \mathrm{H} & -0.375227 & -2.121941 & 1.881188 \\ \mathrm{H} & -2.015406 & -1.588043 & 2.199345 \\ \mathrm{H} & 1.305246 & -0.996180 & -1.557952 \\ \mathrm{C} & 4.135686 & -0.694673 & -0.743867 \\ \mathrm{H} & 4.943820 & -0.037853 & -1.040832 \\ \mathrm{H} & 4.476280 & -1.266101 & 0.111445 \\ \mathrm{H} & 3.962829 & -1.393560 & -1.552501\end{array}$


$1-Z, S_{0}$ geometry

CASSCF/cc-pVTZ S $\mathrm{S}_{0}$ energy: -518.96623394

$\begin{array}{lrrr}\mathrm{C} & -0.71575957 & -0.01383721 & -0.13881212 \\ \mathrm{C} & 0.62987589 & -0.20097539 & -0.11160125 \\ \mathrm{C} & 1.27655587 & -1.51326034 & -0.22938278 \\ \mathrm{C} & 1.74132571 & 0.75387457 & 0.00382530 \\ \mathrm{C} & 2.91583922 & 0.08115815 & -0.04108589 \\ \mathrm{C} & 2.61682211 & -1.34715808 & -0.18710798 \\ \mathrm{~N} & -1.42060552 & 1.16055816 & -0.06503045 \\ \mathrm{C} & -2.85324578 & 0.96301281 & -0.11906797 \\ \mathrm{H} & 3.35372380 & -2.12305684 & -0.25380308 \\ \mathrm{C} & -1.72761733 & -1.14540431 & -0.25671208 \\ \mathrm{C} & -2.98398344 & -0.41662202 & -0.74814950 \\ \mathrm{H} & -2.96089148 & -0.32427167 & -1.82709098 \\ \mathrm{H} & -3.90090036 & -0.91996011 & -0.47235843 \\ \mathrm{H} & -3.30069204 & 0.99827813 & 0.87362989 \\ \mathrm{H} & -3.32366198 & 1.73737853 & -0.71281268 \\ \mathrm{H} & -1.40051857 & -1.87770478 & -0.97959545 \\ \mathrm{H} & 0.76651852 & -2.44707410 & -0.33854150 \\ \mathrm{C} & -0.89255480 & 2.42256144 & 0.37554766 \\ \mathrm{H} & -0.33692741 & 2.32581701 & 1.30228392 \\ \mathrm{H} & -0.24426237 & 2.87117667 & -0.36752716 \\ \mathrm{H} & -1.71550899 & 3.10220044 & 0.54719433 \\ \mathrm{C} & -1.95734465 & -1.84367379 & 1.08809106 \\ \mathrm{H} & -2.70819107 & -2.61953456 & 0.98465343 \\ \mathrm{H} & -1.04729533 & -2.29778327 & 1.45494219 \\ \mathrm{H} & -2.30166491 & -1.14450401 & 1.84218241 \\ \mathrm{H} & 1.65554339 & 1.81331763 & 0.10146355 \\ \mathrm{C} & 4.29851060 & 0.65142732 & 0.03559290 \\ \mathrm{H} & 4.27344861 & 1.72901641 & 0.13866823 \\ \mathrm{H} & 4.84317401 & 0.24685683 & 0.88303772 \\ \mathrm{H} & 4.87053087 & 0.41394133 & -0.85595130\end{array}$


1-Z, MEP point $1\left(\mathrm{~S}_{2}\right)$

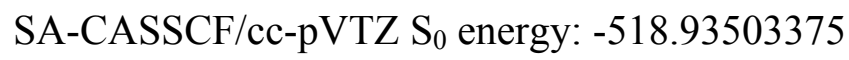

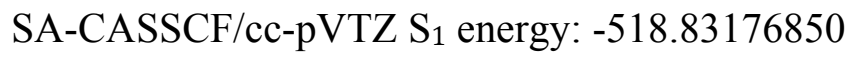

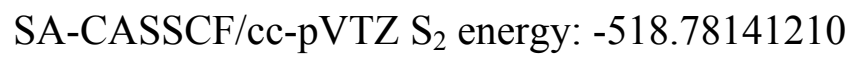

$\begin{array}{lrrr}\mathrm{C} & -0.786141 & -0.019295 & -0.037771 \\ \mathrm{C} & 0.692863 & -0.217417 & -0.077746 \\ \mathrm{C} & 1.263920 & -1.495746 & -0.251673 \\ \mathrm{C} & 1.722376 & 0.744918 & 0.021622 \\ \mathrm{C} & 2.965182 & 0.059832 & -0.060505 \\ \mathrm{C} & 2.676768 & -1.339018 & -0.225635 \\ \mathrm{~N} & -1.415401 & 1.159085 & -0.069692 \\ \mathrm{C} & -2.859479 & 0.994593 & -0.165279 \\ \mathrm{H} & 3.401571 & -2.125451 & -0.275264 \\ \mathrm{C} & -1.760716 & -1.139108 & -0.231053 \\ \mathrm{C} & -3.015469 & -0.409021 & -0.734412 \\ \mathrm{H} & -3.007508 & -0.362650 & -1.815895 \\ \mathrm{H} & -3.937702 & -0.880146 & -0.424898 \\ \mathrm{H} & -3.303081 & 1.091372 & 0.823082 \\ \mathrm{H} & -3.282357 & 1.766298 & -0.793789 \\ \mathrm{H} & -1.391109 & -1.831700 & -0.976737 \\ \mathrm{H} & 0.741630 & -2.421605 & -0.359858 \\ \mathrm{C} & -0.887177 & 2.442814 & 0.339984 \\ \mathrm{H} & -0.344877 & 2.359862 & 1.271374 \\ \mathrm{H} & -0.232156 & 2.854779 & -0.412912 \\ \mathrm{H} & -1.717537 & 3.118649 & 0.479701 \\ \mathrm{C} & -1.991559 & -1.907348 & 1.078775 \\ \mathrm{H} & -2.676503 & -2.732191 & 0.914642 \\ \mathrm{H} & -1.062961 & -2.305872 & 1.462022 \\ \mathrm{H} & -2.417597 & -1.261963 & 1.839246 \\ \mathrm{H} & 1.629036 & 1.798875 & 0.151512 \\ \mathrm{C} & 4.326471 & 0.670647 & 0.040293 \\ \mathrm{H} & 4.265646 & 1.750398 & 0.113022 \\ \mathrm{H} & 4.865979 & 0.314387 & 0.914108 \\ \mathrm{H} & 4.936130 & 0.434056 & -0.827407\end{array}$


1-Z, MEP point $2\left(\mathrm{~S}_{2}\right)$

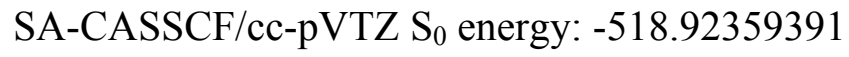

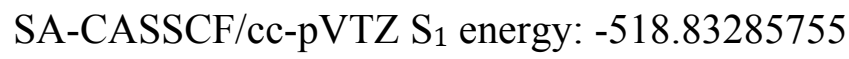

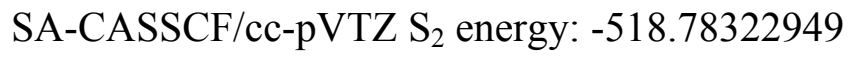

$\begin{array}{lrrc}\mathrm{C} & -0.803144 & -0.019837 & 0.081169 \\ \mathrm{C} & 0.699247 & -0.220471 & -0.024136 \\ \mathrm{C} & 1.268103 & -1.474406 & -0.327466 \\ \mathrm{C} & 1.733592 & 0.733318 & 0.108027 \\ \mathrm{C} & 2.966759 & 0.065650 & -0.065693 \\ \mathrm{C} & 2.674384 & -1.317637 & -0.318300 \\ \mathrm{~N} & -1.402693 & 1.158763 & -0.093698 \\ \mathrm{C} & -2.847198 & 1.017900 & -0.229049 \\ \mathrm{H} & 3.396947 & -2.103767 & -0.403896 \\ \mathrm{C} & -1.770417 & -1.139766 & -0.175008 \\ \mathrm{C} & -3.019660 & -0.415236 & -0.711436 \\ \mathrm{H} & -3.022903 & -0.434623 & -1.793295 \\ \mathrm{H} & -3.945038 & -0.855148 & -0.367623 \\ \mathrm{H} & -3.315487 & 1.189900 & 0.737421 \\ \mathrm{H} & -3.230885 & 1.757292 & -0.918172 \\ \mathrm{H} & -1.363059 & -1.794965 & -0.935310 \\ \mathrm{H} & 0.745632 & -2.395225 & -0.476240 \\ \mathrm{C} & -0.873423 & 2.457853 & 0.273513 \\ \mathrm{H} & -0.376918 & 2.413291 & 1.232540 \\ \mathrm{H} & -0.178680 & 2.819519 & -0.468103 \\ \mathrm{H} & -1.699238 & 3.150040 & 0.341331 \\ \mathrm{C} & -2.025747 & -1.968545 & 1.088346 \\ \mathrm{H} & -2.673955 & -2.807730 & 0.861870 \\ \mathrm{H} & -1.098308 & -2.353633 & 1.489505 \\ \mathrm{H} & -2.502830 & -1.371716 & 1.857993 \\ \mathrm{H} & 1.643738 & 1.773554 & 0.325911 \\ \mathrm{C} & 4.330485 & 0.673535 & 0.053590 \\ \mathrm{H} & 4.272034 & 1.755417 & 0.090854 \\ \mathrm{H} & 4.846118 & 0.344672 & 0.952509 \\ \mathrm{H} & 4.960238 & 0.407226 & -0.789968\end{array}$


1-Z, MEP point $3\left(\mathrm{~S}_{2}\right)$

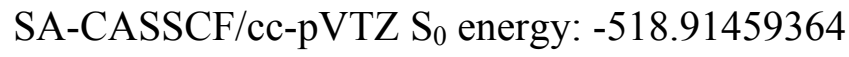

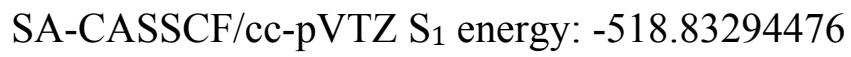

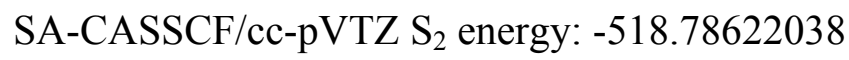

$\begin{array}{lrrc}\mathrm{C} & -0.811643 & -0.017048 & 0.147038 \\ \mathrm{C} & 0.698422 & -0.222285 & 0.021825 \\ \mathrm{C} & 1.257213 & -1.433990 & -0.435997 \\ \mathrm{C} & 1.739882 & 0.710094 & 0.232116 \\ \mathrm{C} & 2.960178 & 0.068812 & -0.047810 \\ \mathrm{C} & 2.658560 & -1.280626 & -0.431106 \\ \mathrm{~N} & -1.389292 & 1.152154 & -0.123624 \\ \mathrm{C} & -2.832560 & 1.023775 & -0.285667 \\ \mathrm{H} & 3.376789 & -2.059907 & -0.588839 \\ \mathrm{C} & -1.772058 & -1.143674 & -0.117172 \\ \mathrm{C} & -3.017649 & -0.432834 & -0.684579 \\ \mathrm{H} & -3.030744 & -0.514280 & -1.763228 \\ \mathrm{H} & -3.944458 & -0.843688 & -0.310095 \\ \mathrm{H} & -3.317776 & 1.261842 & 0.658018 \\ \mathrm{H} & -3.188858 & 1.725880 & -1.026335 \\ \mathrm{H} & -1.345528 & -1.786994 & -0.876410 \\ \mathrm{H} & 0.732735 & -2.337969 & -0.661183 \\ \mathrm{C} & -0.855971 & 2.462711 & 0.200255 \\ \mathrm{H} & -0.435530 & 2.467671 & 1.196655 \\ \mathrm{H} & -0.097541 & 2.760131 & -0.505177 \\ \mathrm{H} & -1.667091 & 3.174173 & 0.162162 \\ \mathrm{C} & -2.038836 & -1.991822 & 1.127281 \\ \mathrm{H} & -2.678737 & -2.830423 & 0.877338 \\ \mathrm{H} & -1.113908 & -2.380054 & 1.532172 \\ \mathrm{H} & -2.529373 & -1.411837 & 1.901113 \\ \mathrm{H} & 1.655225 & 1.721498 & 0.563220 \\ \mathrm{C} & 4.328892 & 0.663736 & 0.081895 \\ \mathrm{H} & 4.281248 & 1.746294 & 0.120355 \\ \mathrm{H} & 4.836874 & 0.329318 & 0.983233 \\ \mathrm{H} & 4.958767 & 0.391734 & -0.759063\end{array}$


1-Z, MEP point $4\left(\mathrm{~S}_{2}\right)$

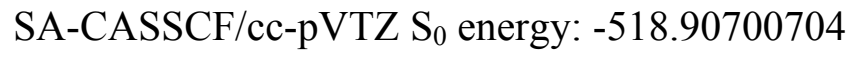

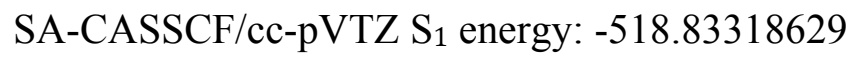

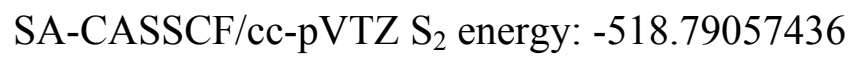

$\begin{array}{lrrc}\mathrm{C} & -0.816221 & -0.016415 & 0.180428 \\ \mathrm{C} & 0.696049 & -0.228667 & 0.052083 \\ \mathrm{C} & 1.246876 & -1.379244 & -0.553409 \\ \mathrm{C} & 1.745445 & 0.669388 & 0.356182 \\ \mathrm{C} & 2.956259 & 0.068073 & -0.018872 \\ \mathrm{C} & 2.645018 & -1.232119 & -0.540368 \\ \mathrm{~N} & -1.378217 & 1.141544 & -0.157773 \\ \mathrm{C} & -2.821392 & 1.023590 & -0.330847 \\ \mathrm{H} & 3.358954 & -1.996358 & -0.774541 \\ \mathrm{C} & -1.778630 & -1.148285 & -0.064931 \\ \mathrm{C} & -3.024055 & -0.450182 & -0.651474 \\ \mathrm{H} & -3.054680 & -0.587937 & -1.723850 \\ \mathrm{H} & -3.949422 & -0.831053 & -0.243332 \\ \mathrm{H} & -3.313411 & 1.320495 & 0.592213 \\ \mathrm{H} & -3.159689 & 1.688543 & -1.113048 \\ \mathrm{H} & -1.347800 & -1.794333 & -0.818590 \\ \mathrm{H} & 0.718920 & -2.255355 & -0.864524 \\ \mathrm{C} & -0.836393 & 2.458766 & 0.127496 \\ \mathrm{H} & -0.505620 & 2.517790 & 1.156421 \\ \mathrm{H} & -0.009701 & 2.689891 & -0.522428 \\ \mathrm{H} & -1.619628 & 3.185110 & -0.027651 \\ \mathrm{C} & -2.043401 & -1.994741 & 1.178475 \\ \mathrm{H} & -2.678370 & -2.836729 & 0.927513 \\ \mathrm{H} & -1.116527 & -2.379326 & 1.583147 \\ \mathrm{H} & -2.537572 & -1.417699 & 1.952153 \\ \mathrm{H} & 1.659906 & 1.640902 & 0.793589 \\ \mathrm{C} & 4.329155 & 0.652663 & 0.122696 \\ \mathrm{H} & 4.287259 & 1.733902 & 0.194134 \\ \mathrm{H} & 4.841874 & 0.289352 & 1.010033 \\ \mathrm{H} & 4.950904 & 0.403371 & -0.730785\end{array}$


1-Z, MEP point $5\left(\mathrm{~S}_{2}\right)$

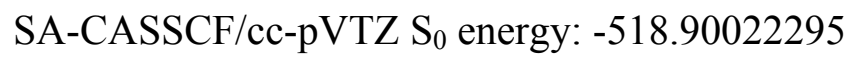

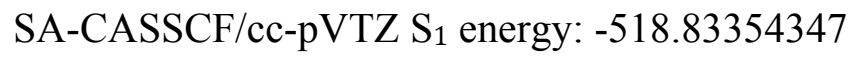

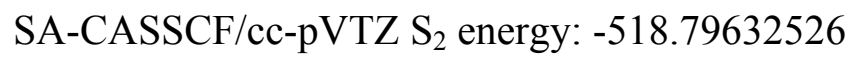

$\begin{array}{lrrc}\mathrm{C} & -0.816461 & -0.017786 & 0.196789 \\ \mathrm{C} & 0.691839 & -0.238256 & 0.071914 \\ \mathrm{C} & 1.236880 & -1.311426 & -0.670532 \\ \mathrm{C} & 1.748248 & 0.615164 & 0.471146 \\ \mathrm{C} & 2.950839 & 0.064215 & 0.012655 \\ \mathrm{C} & 2.631573 & -1.172443 & -0.642248 \\ \mathrm{~N} & -1.366493 & 1.127869 & -0.195285 \\ \mathrm{C} & -2.810499 & 1.019519 & -0.367458 \\ \mathrm{H} & 3.341939 & -1.913297 & -0.951306 \\ \mathrm{C} & -1.785206 & -1.152865 & -0.015415 \\ \mathrm{C} & -3.032012 & -0.466047 & -0.614823 \\ \mathrm{H} & -3.082627 & -0.654789 & -1.678603 \\ \mathrm{H} & -3.954564 & -0.816530 & -0.174162 \\ \mathrm{H} & -3.301452 & 1.368833 & 0.537608 \\ \mathrm{H} & -3.138453 & 1.648234 & -1.183228 \\ \mathrm{H} & -1.357140 & -1.810002 & -0.760650 \\ \mathrm{H} & 0.705216 & -2.149166 & -1.069271 \\ \mathrm{C} & -0.814477 & 2.448391 & 0.056205 \\ \mathrm{H} & -0.576229 & 2.563323 & 1.107143 \\ \mathrm{H} & 0.074349 & 2.617202 & -0.526210 \\ \mathrm{H} & -1.558827 & 3.182225 & -0.213675 \\ \mathrm{C} & -2.044803 & -1.987307 & 1.235070 \\ \mathrm{H} & -2.680117 & -2.831223 & 0.992437 \\ \mathrm{H} & -1.115970 & -2.369472 & 1.637898 \\ \mathrm{H} & -2.536156 & -1.405261 & 2.006594 \\ \mathrm{H} & 1.659722 & 1.538757 & 1.004768 \\ \mathrm{C} & 4.326698 & 0.639460 & 0.170733 \\ \mathrm{H} & 4.288773 & 1.716712 & 0.289846 \\ \mathrm{H} & 4.846361 & 0.236653 & 1.036601 \\ \mathrm{H} & 4.939235 & 0.426526 & -0.698712\end{array}$


1-Z, MEP point $6\left(\mathrm{~S}_{2}\right)$

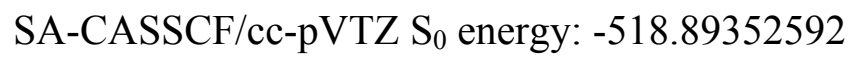

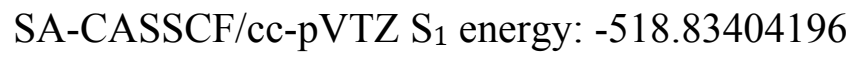

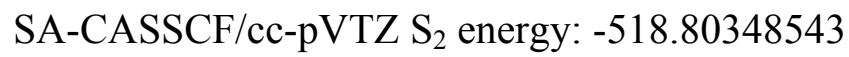

$\begin{array}{lrrc}\mathrm{C} & -0.813468 & -0.021273 & 0.203084 \\ \mathrm{C} & 0.686212 & -0.248374 & 0.082897 \\ \mathrm{C} & 1.227383 & -1.230041 & -0.783238 \\ \mathrm{C} & 1.749420 & 0.548680 & 0.574922 \\ \mathrm{C} & 2.945067 & 0.056209 & 0.044818 \\ \mathrm{C} & 2.618709 & -1.103248 & -0.736770 \\ \mathrm{~N} & -1.355095 & 1.111593 & -0.233116 \\ \mathrm{C} & -2.800156 & 1.012656 & -0.398979 \\ \mathrm{H} & 3.326529 & -1.811484 & -1.119931 \\ \mathrm{C} & -1.791772 & -1.157494 & 0.032062 \\ \mathrm{C} & -3.040741 & -0.480660 & -0.576278 \\ \mathrm{H} & -3.113958 & -0.716650 & -1.629151 \\ \mathrm{H} & -3.958635 & -0.800070 & -0.103542 \\ \mathrm{H} & -3.286556 & 1.410304 & 0.488283 \\ \mathrm{H} & -3.122041 & 1.605712 & -1.243467 \\ \mathrm{H} & -1.369227 & -1.829858 & -0.702546 \\ \mathrm{H} & 0.689682 & -2.018486 & -1.266890 \\ \mathrm{C} & -0.791771 & 2.433329 & -0.013910 \\ \mathrm{H} & -0.642267 & 2.605514 & 1.046262 \\ \mathrm{H} & 0.148141 & 2.545592 & -0.523948 \\ \mathrm{H} & -1.489517 & 3.165662 & -0.390463 \\ \mathrm{C} & -2.044236 & -1.973055 & 1.294569 \\ \mathrm{H} & -2.682025 & -2.819260 & 1.066435 \\ \mathrm{H} & -1.113203 & -2.352119 & 1.695630 \\ \mathrm{H} & -2.529869 & -1.381038 & 2.062144 \\ \mathrm{H} & 1.656223 & 1.416545 & 1.196216 \\ \mathrm{C} & 4.322774 & 0.623120 & 0.223542 \\ \mathrm{H} & 4.286550 & 1.693499 & 0.394235 \\ \mathrm{H} & 4.848778 & 0.179583 & 1.065149 \\ \mathrm{H} & 4.928075 & 0.452459 & -0.659912\end{array}$




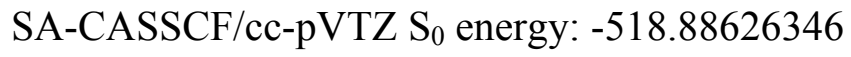

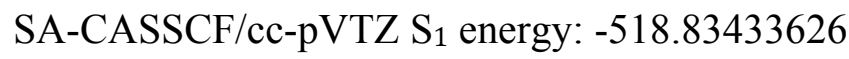

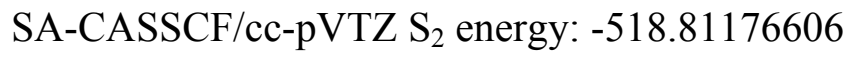

$\begin{array}{lrrc}\mathrm{C} & -0.808214 & -0.026947 & 0.200460 \\ \mathrm{C} & 0.679672 & -0.257081 & 0.085545 \\ \mathrm{C} & 1.219065 & -1.135749 & -0.889816 \\ \mathrm{C} & 1.749781 & 0.471639 & 0.667233 \\ \mathrm{C} & 2.939384 & 0.042615 & 0.077334 \\ \mathrm{C} & 2.607234 & -1.025696 & -0.823259 \\ \mathrm{~N} & -1.344977 & 1.093050 & -0.272311 \\ \mathrm{C} & -2.791535 & 1.002868 & -0.424352 \\ \mathrm{H} & 3.312376 & -1.692215 & -1.279678 \\ \mathrm{C} & -1.797959 & -1.161938 & 0.078472 \\ \mathrm{C} & -3.048755 & -0.494524 & -0.538100 \\ \mathrm{H} & -3.140430 & -0.771892 & -1.579439 \\ \mathrm{H} & -3.962791 & -0.785095 & -0.039704 \\ \mathrm{H} & -3.268693 & 1.441802 & 0.448405 \\ \mathrm{H} & -3.114012 & 1.563156 & -1.290761 \\ \mathrm{H} & -1.383387 & -1.853193 & -0.643435 \\ \mathrm{H} & 0.674936 & -1.865400 & -1.453307 \\ \mathrm{C} & -0.769961 & 2.414548 & -0.082807 \\ \mathrm{H} & -0.699421 & 2.641705 & 0.976259 \\ \mathrm{H} & 0.209182 & 2.478592 & -0.521806 \\ \mathrm{H} & -1.418201 & 3.139624 & -0.551784 \\ \mathrm{C} & -2.042577 & -1.952297 & 1.357119 \\ \mathrm{H} & -2.685131 & -2.799640 & 1.148226 \\ \mathrm{H} & -1.109549 & -2.328674 & 1.756268 \\ \mathrm{H} & -2.519660 & -1.345803 & 2.118645 \\ \mathrm{H} & 1.653519 & 1.278091 & 1.367291 \\ \mathrm{C} & 4.317772 & 0.602737 & 0.279345 \\ \mathrm{H} & 4.281307 & 1.663859 & 0.500918 \\ \mathrm{H} & 4.848980 & 0.120683 & 1.095933 \\ \mathrm{H} & 4.917954 & 0.475928 & -0.614638\end{array}$


1-Z, MEP point $8\left(\mathrm{~S}_{2}\right)$

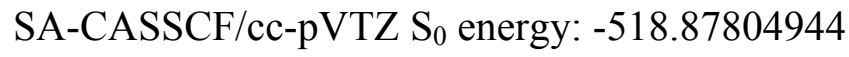

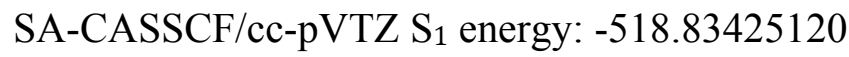

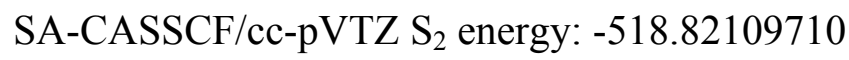

$\begin{array}{lrrc}\mathrm{C} & -0.801287 & -0.034885 & 0.192362 \\ \mathrm{C} & 0.672775 & -0.264339 & 0.081612 \\ \mathrm{C} & 1.212056 & -1.029868 & -0.987480 \\ \mathrm{C} & 1.749765 & 0.385816 & 0.745832 \\ \mathrm{C} & 2.934114 & 0.024933 & 0.107234 \\ \mathrm{C} & 2.597349 & -0.940443 & -0.901340 \\ \mathrm{~N} & -1.336586 & 1.072863 & -0.310109 \\ \mathrm{C} & -2.784302 & 0.991104 & -0.446264 \\ \mathrm{H} & 3.300680 & -1.556500 & -1.426297 \\ \mathrm{C} & -1.803476 & -1.166004 & 0.122869 \\ \mathrm{C} & -3.057197 & -0.507602 & -0.498715 \\ \mathrm{H} & -3.169704 & -0.824036 & -1.526622 \\ \mathrm{H} & -3.965359 & -0.769170 & 0.025773 \\ \mathrm{H} & -3.251051 & 1.468909 & 0.411390 \\ \mathrm{H} & -3.109497 & 1.518975 & -1.332025 \\ \mathrm{H} & -1.398599 & -1.876997 & -0.585370 \\ \mathrm{H} & 0.661397 & -1.694600 & -1.622411 \\ \mathrm{C} & -0.750779 & 2.393278 & -0.151254 \\ \mathrm{H} & -0.759535 & 2.678854 & 0.896407 \\ \mathrm{H} & 0.261043 & 2.413274 & -0.513318 \\ \mathrm{H} & -1.343128 & 3.102572 & -0.710329 \\ \mathrm{C} & -2.038642 & -1.926912 & 1.419933 \\ \mathrm{H} & -2.687235 & -2.775350 & 1.234982 \\ \mathrm{H} & -1.103480 & -2.300130 & 1.817445 \\ \mathrm{H} & -2.505439 & -1.302876 & 2.174033 \\ \mathrm{H} & 1.650968 & 1.123809 & 1.517749 \\ \mathrm{C} & 4.312409 & 0.576713 & 0.337908 \\ \mathrm{H} & 4.274789 & 1.626178 & 0.609350 \\ \mathrm{H} & 4.845269 & 0.057696 & 1.130356 \\ \mathrm{H} & 4.911694 & 0.494017 & -0.561496\end{array}$


1-Z, MEP point $9\left(\mathrm{~S}_{2}\right)$

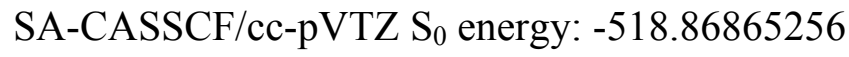

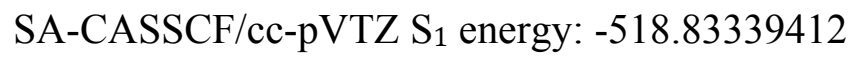

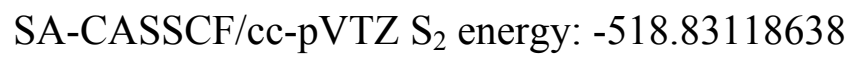

$\begin{array}{lrrc}\mathrm{C} & -0.793379 & -0.045121 & 0.177760 \\ \mathrm{C} & 0.665891 & -0.271342 & 0.071987 \\ \mathrm{C} & 1.207646 & -0.914584 & -1.074479 \\ \mathrm{C} & 1.749273 & 0.293521 & 0.810790 \\ \mathrm{C} & 2.928393 & 0.004152 & 0.134322 \\ \mathrm{C} & 2.590410 & -0.848179 & -0.970460 \\ \mathrm{~N} & -1.330747 & 1.051540 & -0.346740 \\ \mathrm{C} & -2.779246 & 0.977648 & -0.464590 \\ \mathrm{H} & 3.293266 & -1.403111 & -1.560206 \\ \mathrm{C} & -1.808580 & -1.169510 & 0.166175 \\ \mathrm{C} & -3.065785 & -0.519986 & -0.458637 \\ \mathrm{H} & -3.198614 & -0.872279 & -1.472473 \\ \mathrm{H} & -3.967517 & -0.753726 & 0.089555 \\ \mathrm{H} & -3.234208 & 1.491094 & 0.378884 \\ \mathrm{H} & -3.110257 & 1.474353 & -1.366196 \\ \mathrm{H} & -1.415791 & -1.901575 & -0.527841 \\ \mathrm{H} & 0.652287 & -1.503996 & -1.776842 \\ \mathrm{C} & -0.734230 & 2.370267 & -0.219938 \\ \mathrm{H} & -0.810603 & 2.711973 & 0.808758 \\ \mathrm{H} & 0.299778 & 2.353128 & -0.511113 \\ \mathrm{H} & -1.271987 & 3.058502 & -0.855481 \\ \mathrm{C} & -2.032522 & -1.897166 & 1.483257 \\ \mathrm{H} & -2.688626 & -2.745036 & 1.324543 \\ \mathrm{H} & -1.095312 & -2.267892 & 1.878180 \\ \mathrm{H} & -2.486725 & -1.253455 & 2.228039 \\ \mathrm{H} & 1.648272 & 0.954599 & 1.649785 \\ \mathrm{C} & 4.306828 & 0.544255 & 0.398393 \\ \mathrm{H} & 4.268283 & 1.580837 & 0.715672 \\ \mathrm{H} & 4.837254 & -0.009489 & 1.167954 \\ \mathrm{H} & 4.908493 & 0.503042 & -0.502262\end{array}$


1-Z, MEP point $10\left(\mathrm{~S}_{1}\right)$

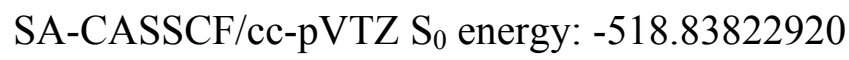

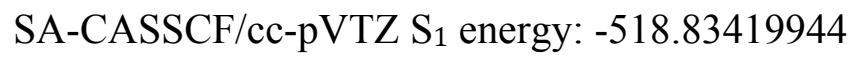

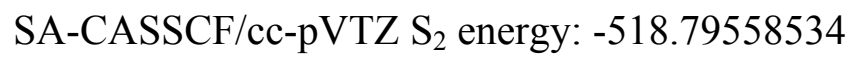

$\begin{array}{lrrr}\mathrm{C} & -0.791976 & -0.057909 & 0.219246 \\ \mathrm{C} & 0.648641 & -0.268375 & 0.055898 \\ \mathrm{C} & 1.189785 & -0.931548 & -1.043189 \\ \mathrm{C} & 1.719201 & 0.299056 & 0.793070 \\ \mathrm{C} & 2.957797 & -0.021286 & 0.101191 \\ \mathrm{C} & 2.633674 & -0.828529 & -0.955200 \\ \mathrm{~N} & -1.341109 & 1.074484 & -0.373485 \\ \mathrm{C} & -2.775758 & 0.983772 & -0.453772 \\ \mathrm{H} & 3.321349 & -1.301613 & -1.625545 \\ \mathrm{C} & -1.802294 & -1.178292 & 0.152154 \\ \mathrm{C} & -3.063217 & -0.519996 & -0.456816 \\ \mathrm{H} & -3.207289 & -0.868114 & -1.472383 \\ \mathrm{H} & -3.965067 & -0.758767 & 0.093357 \\ \mathrm{H} & -3.263290 & 1.474618 & 0.392815 \\ \mathrm{H} & -3.132341 & 1.474941 & -1.353201 \\ \mathrm{H} & -1.442879 & -1.935190 & -0.539753 \\ \mathrm{H} & 0.645415 & -1.465132 & -1.791157 \\ \mathrm{C} & -0.743211 & 2.365885 & -0.216964 \\ \mathrm{H} & -0.801740 & 2.740733 & 0.809065 \\ \mathrm{H} & 0.296352 & 2.346953 & -0.511086 \\ \mathrm{H} & -1.257465 & 3.071849 & -0.857744 \\ \mathrm{C} & -2.033515 & -1.894035 & 1.483465 \\ \mathrm{H} & -2.695312 & -2.741247 & 1.340162 \\ \mathrm{H} & -1.096692 & -2.264218 & 1.884143 \\ \mathrm{H} & -2.477496 & -1.236779 & 2.222713 \\ \mathrm{H} & 1.634609 & 0.918444 & 1.662671 \\ \mathrm{C} & 4.312571 & 0.539031 & 0.405912 \\ \mathrm{H} & 4.251616 & 1.576207 & 0.714651 \\ \mathrm{H} & 4.827481 & -0.003307 & 1.191453 \\ \mathrm{H} & 4.932293 & 0.499610 & -0.481759\end{array}$


2-E, $\mathrm{S}_{0}$ geometry

CASSCF/cc-pVTZ $\mathrm{S}_{0}$ energy: -518.96072038

$\begin{array}{lrrr}\text { C } & -0.99383791 & -0.39280169 & 0.02344371 \\ \text { C } & 0.32583112 & -0.11057166 & -0.03904560 \\ \text { C } & 1.02291189 & 1.24924852 & -0.06235021 \\ \text { C } & 1.38950427 & -1.12137353 & -0.00581271 \\ \text { C } & 2.60601994 & -0.58212598 & -0.16059197 \\ \text { C } & 2.50568663 & 0.91053124 & -0.35079658 \\ \text { N } & -2.09236447 & 0.49902195 & 0.00914270 \\ \text { C } & -3.33745901 & -0.25841902 & 0.01772843 \\ \text { H } & 2.78875832 & 1.18066285 & -1.36515818 \\ \text { C } & -1.56192358 & -1.73287163 & 0.22396359 \\ \text { C } & -2.89445957 & -1.66889709 & 0.25374614 \\ \text { H } & -3.86414100 & -0.17177625 & -0.93315167 \\ \text { H } & -4.01111945 & 0.10519719 & 0.78858191 \\ \text { H } & 0.63955665 & 1.87940525 & -0.85137625 \\ \text { C } & -2.10381655 & 1.66052736 & -0.84541000 \\ \text { H } & -1.92972307 & 1.41306467 & -1.89308910 \\ \text { H } & -1.37043843 & 2.38908979 & -0.54072187 \\ \text { H } & -3.07289087 & 2.13582306 & -0.76945693 \\ \text { H } & 1.21423871 & -2.17220105 & 0.11307945 \\ \text { H } & 3.17716373 & 1.45104046 & 0.30942802 \\ \text { H } & -0.97504151 & -2.61559347 & 0.35981521 \\ \text { H } & -3.57220212 & -2.48423662 & 0.40482322 \\ \text { C } & 0.86306955 & 1.97957410 & 1.27414680 \\ \text { H } & -0.17915224 & 2.14383831 & 1.51589377 \\ \text { H } & 1.36421664 & 2.94191508 & 1.24994830 \\ \text { H } & 1.29760421 & 1.39532989 & 2.07814990 \\ \text { C } & 3.91820333 & -1.29943287 & -0.20567416 \\ \text { H } & 3.79314966 & -2.36680709 & -0.07424001 \\ \text { H } & 4.41909478 & -1.13316598 & -1.15509436 \\ \text { H } & 4.58549238 & -0.93973177 & 0.57188047\end{array}$


2-E, MEP point $1\left(\mathrm{~S}_{1}\right)$

SA-CASSCF/cc-pVTZ S $\mathrm{S}_{0}$ energy: -518.91948028

SA-CASSCF/cc-pVTZ S $S_{1}$ energy: -518.79493142

$\begin{array}{lrrc}\text { C } & -1.043223 & -0.446356 & 0.034692 \\ \text { C } & 0.365993 & -0.105818 & -0.085571 \\ \text { C } & 1.036807 & 1.262557 & -0.053832 \\ \text { C } & 1.339653 & -1.089948 & -0.055247 \\ \text { C } & 2.647510 & -0.552761 & -0.134553 \\ \text { C } & 2.525594 & 0.930641 & -0.339152 \\ \text { N } & -2.077818 & 0.447722 & -0.051950 \\ \text { C } & -3.349374 & -0.258249 & -0.001991 \\ \text { H } & 2.785633 & 1.186185 & -1.365522 \\ \text { C } & -1.535732 & -1.748612 & 0.251112 \\ \text { C } & -2.990685 & -1.660888 & 0.328829 \\ \text { H } & -3.840403 & -0.189829 & -0.972441 \\ \text { H } & -4.009603 & 0.204742 & 0.726282 \\ \text { H } & 0.667364 & 1.911843 & -0.835963 \\ \text { C } & -2.076095 & 1.668272 & -0.826715 \\ \text { H } & -1.909033 & 1.476730 & -1.883921 \\ \text { H } & -1.336088 & 2.366625 & -0.478155 \\ \text { H } & -3.043689 & 2.137557 & -0.717182 \\ \text { H } & 1.143327 & -2.140966 & 0.005558 \\ \text { H } & 3.196678 & 1.499343 & 0.297524 \\ \text { H } & -0.951845 & -2.577001 & 0.579871 \\ \text { H } & -3.680481 & -2.473362 & 0.237165 \\ \text { C } & 0.866568 & 1.977502 & 1.289959 \\ \text { H } & -0.176944 & 2.136130 & 1.533480 \\ \text { H } & 1.360537 & 2.943967 & 1.276898 \\ \text { H } & 1.301550 & 1.388335 & 2.089930 \\ \text { C } & 3.932580 & -1.302250 & -0.211649 \\ \text { H } & 3.781736 & -2.364882 & -0.066005 \\ \text { H } & 4.417754 & -1.165330 & -1.176309 \\ \text { H } & 4.635682 & -0.960082 & 0.543290\end{array}$


2-E, MEP point $2\left(\mathrm{~S}_{1}\right)$

SA-CASSCF/cc-pVTZ S $\mathrm{S}_{0}$ energy: -518.91336875

SA-CASSCF/cc-pVTZ S $S_{1}$ energy: -518.79704023

$\begin{array}{lrrr}\text { C } & -1.044358 & -0.450136 & 0.039961 \\ \text { C } & 0.359496 & -0.098732 & -0.136201 \\ \text { C } & 1.035451 & 1.269888 & -0.031609 \\ \text { C } & 1.342297 & -1.080152 & -0.125091 \\ \text { C } & 2.641571 & -0.546118 & -0.147189 \\ \text { C } & 2.528407 & 0.945069 & -0.306267 \\ \text { N } & -2.072174 & 0.415177 & -0.143830 \\ \text { C } & -3.348113 & -0.268052 & -0.045484 \\ \text { H } & 2.800521 & 1.227887 & -1.322285 \\ \text { C } & -1.543684 & -1.731552 & 0.377281 \\ \text { C } & -3.002331 & -1.645925 & 0.396616 \\ \text { H } & -3.832528 & -0.268352 & -1.020605 \\ \text { H } & -4.004023 & 0.258803 & 0.642512 \\ \text { H } & 0.681798 & 1.955245 & -0.790137 \\ \text { C } & -2.044421 & 1.677465 & -0.841694 \\ \text { H } & -1.789526 & 1.554448 & -1.890034 \\ \text { H } & -1.352806 & 2.369585 & -0.394246 \\ \text { H } & -3.030581 & 2.115423 & -0.781653 \\ \text { H } & 1.138556 & -2.132326 & -0.106251 \\ \text { H } & 3.192167 & 1.495346 & 0.353766 \\ \text { H } & -0.963097 & -2.508388 & 0.820578 \\ \text { H } & -3.680645 & -2.464866 & 0.260978 \\ \text { C } & 0.853726 & 1.932110 & 1.337880 \\ \text { H } & -0.191186 & 2.088473 & 1.578455 \\ \text { H } & 1.352147 & 2.895910 & 1.366421 \\ \text { H } & 1.277550 & 1.311132 & 2.119468 \\ \text { C } & 3.928540 & -1.295765 & -0.236732 \\ \text { H } & 3.777825 & -2.358363 & -0.090596 \\ \text { H } & 4.404376 & -1.158630 & -1.206101 \\ \text { H } & 4.638168 & -0.954064 & 0.511741\end{array}$


2-E, MEP point $3\left(\mathrm{~S}_{1}\right)$

SA-CASSCF/cc-pVTZ S $\mathrm{S}_{0}$ energy: -518.90903187

SA-CASSCF/cc-pVTZ S $S_{1}$ energy: -518.79844470

$\begin{array}{lrrc}\text { C } & -1.042972 & -0.433465 & 0.058984 \\ \text { C } & 0.353585 & -0.089384 & -0.170382 \\ \text { C } & 1.031074 & 1.270625 & 0.006426 \\ \text { C } & 1.341139 & -1.075376 & -0.188534 \\ \text { C } & 2.629187 & -0.545824 & -0.187560 \\ \text { C } & 2.525658 & 0.953670 & -0.266571 \\ \text { N } & -2.068028 & 0.395799 & -0.224490 \\ \text { C } & -3.342777 & -0.280367 & -0.080451 \\ \text { H } & 2.814100 & 1.291552 & -1.260783 \\ \text { C } & -1.539010 & -1.671137 & 0.534198 \\ \text { C } & -2.992292 & -1.631350 & 0.439909 \\ \text { H } & -3.833991 & -0.330085 & -1.049359 \\ \text { H } & -3.991966 & 0.282382 & 0.586001 \\ \text { H } & 0.685166 & 1.993788 & -0.720636 \\ \text { C } & -2.019261 & 1.667061 & -0.904542 \\ \text { H } & -1.598093 & 1.573728 & -1.898802 \\ \text { H } & -1.449444 & 2.396595 & -0.351589 \\ \text { H } & -3.029593 & 2.038238 & -0.995848 \\ \text { H } & 1.132540 & -2.127325 & -0.205247 \\ \text { H } & 3.183305 & 1.461802 & 0.432640 \\ \text { H } & -0.951307 & -2.424869 & 1.004538 \\ \text { H } & -3.641635 & -2.471790 & 0.292459 \\ \text { C } & 0.832895 & 1.867064 & 1.403269 \\ \text { H } & -0.215135 & 2.021220 & 1.633432 \\ \text { H } & 1.337273 & 2.824554 & 1.486205 \\ \text { H } & 1.238630 & 1.206104 & 2.161335 \\ \text { C } & 3.919685 & -1.289313 & -0.281127 \\ \text { H } & 3.768694 & -2.357238 & -0.181809 \\ \text { H } & 4.415354 & -1.111328 & -1.233604 \\ \text { H } & 4.612336 & -0.976882 & 0.495656\end{array}$


2-E, MEP point $4\left(\mathrm{~S}_{1}\right)$

SA-CASSCF/cc-pVTZ S $\mathrm{S}_{0}$ energy: -518.90069033

SA-CASSCF/cc-pVTZ S $S_{1}$ energy: -518.80010101

$\begin{array}{lrrc}\text { C } & -1.042171 & -0.410907 & 0.079573 \\ \text { C } & 0.347068 & -0.079045 & -0.192316 \\ \text { C } & 1.028894 & 1.268938 & 0.049834 \\ \text { C } & 1.340008 & -1.069468 & -0.246227 \\ \text { C } & 2.618160 & -0.545191 & -0.225702 \\ \text { C } & 2.523921 & 0.958407 & -0.227230 \\ \text { N } & -2.064563 & 0.374387 & -0.299011 \\ \text { C } & -3.337989 & -0.291810 & -0.103443 \\ \text { H } & 2.824537 & 1.347932 & -1.198485 \\ \text { C } & -1.541554 & -1.587033 & 0.696250 \\ \text { C } & -2.975215 & -1.622300 & 0.463997 \\ \text { H } & -3.853153 & -0.369819 & -1.055845 \\ \text { H } & -3.966931 & 0.293085 & 0.564462 \\ \text { H } & 0.686806 & 2.025607 & -0.645243 \\ \text { C } & -2.001940 & 1.644571 & -0.979441 \\ \text { H } & -1.404984 & 1.578179 & -1.878762 \\ \text { H } & -1.596017 & 2.421352 & -0.346463 \\ \text { H } & -3.006117 & 1.930666 & -1.255689 \\ \text { H } & 1.128116 & -2.120196 & -0.299947 \\ \text { H } & 3.177903 & 1.423465 & 0.504905 \\ \text { H } & -0.937915 & -2.364796 & 1.102969 \\ \text { H } & -3.577184 & -2.491027 & 0.281268 \\ \text { C } & 0.817245 & 1.804623 & 1.468700 \\ \text { H } & -0.233050 & 1.962378 & 1.687605 \\ \text { H } & 1.330390 & 2.751772 & 1.600951 \\ \text { H } & 1.202339 & 1.106813 & 2.204001 \\ \text { C } & 3.910656 & -1.284847 & -0.332493 \\ \text { H } & 3.756657 & -2.356488 & -0.300063 \\ \text { H } & 4.429254 & -1.051486 & -1.260199 \\ \text { H } & 4.584447 & -1.020217 & 0.478123\end{array}$


2-E, MEP point $5\left(\mathrm{~S}_{1}\right)$

SA-CASSCF/cc-pVTZ S $\mathrm{S}_{0}$ energy: -518.89289658

SA-CASSCF/cc-pVTZ S $S_{1}$ energy: -518.80159983

$\begin{array}{lrrc}\text { C } & -1.042212 & -0.384698 & 0.089858 \\ \text { C } & 0.342280 & -0.064658 & -0.202789 \\ \text { C } & 1.030497 & 1.266613 & 0.102875 \\ \text { C } & 1.336973 & -1.060547 & -0.295308 \\ \text { C } & 2.608952 & -0.543165 & -0.256517 \\ \text { C } & 2.524705 & 0.960482 & -0.181893 \\ \text { N } & -2.063640 & 0.351476 & -0.369826 \\ \text { C } & -3.333758 & -0.304664 & -0.124600 \\ \text { H } & 2.834855 & 1.398544 & -1.129112 \\ \text { C } & -1.541499 & -1.505946 & 0.809293 \\ \text { C } & -2.955263 & -1.599872 & 0.511214 \\ \text { H } & -3.862769 & -0.430115 & -1.063059 \\ \text { H } & -3.952858 & 0.310376 & 0.525792 \\ \text { H } & 0.692084 & 2.056683 & -0.557334 \\ \text { C } & -1.997622 & 1.609834 & -1.070616 \\ \text { H } & -1.242250 & 1.577748 & -1.840761 \\ \text { H } & -1.782730 & 2.433852 & -0.400822 \\ \text { H } & -2.954871 & 1.795128 & -1.536388 \\ \text { H } & 1.119756 & -2.107517 & -0.393007 \\ \text { H } & 3.177268 & 1.381504 & 0.577896 \\ \text { H } & -0.928159 & -2.261629 & 1.244530 \\ \text { H } & -3.531478 & -2.491636 & 0.361490 \\ \text { C } & 0.809569 & 1.739181 & 1.542489 \\ \text { H } & -0.241901 & 1.900110 & 1.754947 \\ \text { H } & 1.331173 & 2.673613 & 1.723198 \\ \text { H } & 1.177815 & 1.004735 & 2.250428 \\ \text { C } & 3.900794 & -1.279988 & -0.393675 \\ \text { H } & 3.743419 & -2.351199 & -0.427044 \\ \text { H } & 4.429895 & -0.993286 & -1.300207 \\ \text { H } & 4.566683 & -1.066629 & 0.438387\end{array}$


2-E, MEP point $6\left(\mathrm{~S}_{1}\right)$

SA-CASSCF/cc-pVTZ S $\mathrm{S}_{0}$ energy: -518.88779015

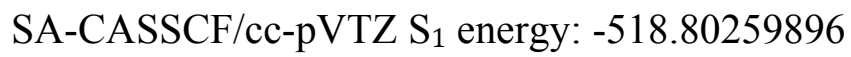

\begin{tabular}{|c|c|c|c|}
\hline $\mathrm{C}$ & -1.043073 & -0.356691 & 0.093971 \\
\hline $\mathrm{C}$ & 0.338724 & -0.044053 & -0.209027 \\
\hline C & 1.035660 & 1.264854 & 0.164260 \\
\hline$C$ & 1.331366 & -1.044913 & -0.340939 \\
\hline $\mathrm{C}$ & 2.601551 & -0.538230 & -0.280107 \\
\hline $\mathrm{C}$ & 2.527664 & 0.962005 & -0.135442 \\
\hline $\mathrm{N}$ & -2.064827 & 0.330829 & -0.427114 \\
\hline $\mathrm{C}$ & -3.330496 & -0.317856 & -0.144020 \\
\hline $\mathrm{H}$ & 2.840307 & 1.442452 & -1.061181 \\
\hline $\mathrm{C}$ & -1.539215 & -1.432478 & 0.884218 \\
\hline $\mathrm{C}$ & -2.939472 & -1.565634 & 0.574283 \\
\hline $\mathrm{H}$ & -3.856877 & -0.506062 & -1.073080 \\
\hline $\mathrm{H}$ & -3.955102 & 0.331493 & 0.466557 \\
\hline $\mathrm{H}$ & 0.700223 & 2.090604 & -0.454358 \\
\hline $\mathrm{C}$ & -2.002286 & 1.558678 & -1.180184 \\
\hline $\mathrm{H}$ & -1.136703 & 1.556648 & -1.821790 \\
\hline $\mathrm{H}$ & -1.959953 & 2.424957 & -0.529290 \\
\hline $\mathrm{H}$ & -2.888290 & 1.638116 & -1.794485 \\
\hline $\mathrm{H}$ & 1.106926 & -2.085446 & -0.484823 \\
\hline $\mathrm{H}$ & 3.183102 & 1.342791 & 0.643005 \\
\hline $\mathrm{H}$ & -0.919453 & -2.143304 & 1.381729 \\
\hline $\mathrm{H}$ & -3.514935 & -2.466976 & 0.504667 \\
\hline $\mathrm{C}$ & 0.813980 & 1.668088 & 1.624860 \\
\hline $\mathrm{H}$ & -0.237169 & 1.829749 & 1.839674 \\
\hline $\mathrm{H}$ & 1.343166 & 2.587936 & 1.852632 \\
\hline $\mathrm{H}$ & 1.171842 & 0.897160 & 2.298340 \\
\hline $\mathrm{C}$ & 3.889897 & -1.273445 & -0.457041 \\
\hline $\mathrm{H}$ & 3.726539 & -2.340502 & -0.548052 \\
\hline $\mathrm{H}$ & 4.420455 & -0.941694 & -1.347304 \\
\hline $\mathrm{H}$ & 4.557845 & -1.109369 & 0.384669 \\
\hline
\end{tabular}


2-E, MEP point $7\left(\mathrm{~S}_{1}\right)$

SA-CASSCF/cc-pVTZ S $\mathrm{S}_{0}$ energy: -518.88343154

SA-CASSCF/cc-pVTZ S S $_{1}$ energy: -518.80362049

$\begin{array}{lrrc}\text { C } & -1.045800 & -0.327110 & 0.093955 \\ \text { C } & 0.334418 & -0.017973 & -0.220464 \\ \text { C } & 1.041506 & 1.261641 & 0.225386 \\ \text { C } & 1.324283 & -1.023683 & -0.388914 \\ \text { C } & 2.594042 & -0.531846 & -0.297362 \\ \text { C } & 2.529626 & 0.963571 & -0.098004 \\ \text { N } & -2.066864 & 0.308061 & -0.478532 \\ \text { C } & -3.328470 & -0.333055 & -0.160954 \\ \text { H } & 2.834364 & 1.475938 & -1.009459 \\ \text { C } & -1.538455 & -1.360567 & 0.942179 \\ \text { C } & -2.922209 & -1.526810 & 0.635339 \\ \text { H } & -3.849954 & -0.583570 & -1.077749 \\ \text { H } & -3.959167 & 0.349009 & 0.405524 \\ \text { H } & 0.708024 & 2.124036 & -0.344589 \\ \text { C } & -2.012510 & 1.502264 & -1.284727 \\ \text { H } & -1.064319 & 1.554543 & -1.791221 \\ \text { H } & -2.145289 & 2.390766 & -0.676331 \\ \text { H } & -2.802780 & 1.471111 & -2.022187 \\ \text { H } & 1.092912 & -2.056287 & -0.576633 \\ \text { H } & 3.193859 & 1.314984 & 0.687101 \\ \text { H } & -0.911246 & -2.026582 & 1.490595 \\ \text { H } & -3.513677 & -2.417135 & 0.688755 \\ \text { C } & 0.830822 & 1.590991 & 1.706419 \\ \text { H } & -0.218114 & 1.747117 & 1.937026 \\ \text { H } & 1.365817 & 2.495676 & 1.976310 \\ \text { H } & 1.189915 & 0.785764 & 2.338267 \\ \text { C } & 3.878733 & -1.265856 & -0.505425 \\ \text { H } & 3.709589 & -2.328125 & -0.635211 \\ \text { H } & 4.409396 & -0.905276 & -1.384617 \\ \text { H } & 4.550798 & -1.137232 & 0.339484\end{array}$


2-E, MEP point $8\left(\mathrm{~S}_{1}\right)$

SA-CASSCF/cc-pVTZ S $\mathrm{S}_{0}$ energy: -518.87569692

SA-CASSCF/cc-pVTZ S $S_{1}$ energy: -518.80614393

$\begin{array}{lrrc}\text { C } & -1.049324 & -0.295069 & 0.090549 \\ \text { C } & 0.331864 & 0.020200 & -0.244920 \\ \text { C } & 1.052147 & 1.252696 & 0.293758 \\ \text { C } & 1.313961 & -0.988781 & -0.471220 \\ \text { C } & 2.586994 & -0.530358 & -0.314477 \\ \text { C } & 2.534743 & 0.958034 & -0.059431 \\ \text { N } & -2.065519 & 0.266270 & -0.536856 \\ \text { C } & -3.325922 & -0.361543 & -0.182282 \\ \text { H } & 2.818051 & 1.500918 & -0.961355 \\ \text { C } & -1.551805 & -1.289376 & 0.992118 \\ \text { C } & -2.895314 & -1.482897 & 0.704038 \\ \text { H } & -3.843806 & -0.684236 & -1.078037 \\ \text { H } & -3.960633 & 0.358401 & 0.329248 \\ \text { H } & 0.728862 & 2.150209 & -0.230684 \\ \text { C } & -2.025594 & 1.451457 & -1.365087 \\ \text { H } & -1.026817 & 1.587433 & -1.735460 \\ \text { H } & -2.330838 & 2.316530 & -0.786904 \\ \text { H } & -2.705355 & 1.334307 & -2.197760 \\ \text { H } & 1.073181 & -2.006333 & -0.724650 \\ \text { H } & 3.216014 & 1.285861 & 0.722067 \\ \text { H } & -0.916789 & -1.904689 & 1.589899 \\ \text { H } & -3.519085 & -2.315791 & 0.948393 \\ \text { C } & 0.846472 & 1.529779 & 1.784753 \\ \text { H } & -0.202289 & 1.685148 & 2.022726 \\ \text { H } & 1.382341 & 2.424980 & 2.084222 \\ \text { H } & 1.203883 & 0.704666 & 2.391142 \\ \text { C } & 3.868769 & -1.266963 & -0.533655 \\ \text { H } & 3.693814 & -2.325466 & -0.689374 \\ \text { H } & 4.410569 & -0.892880 & -1.401749 \\ \text { H } & 4.538383 & -1.164864 & 0.317483\end{array}$


2-E, MEP point $9\left(\mathrm{~S}_{1}\right)$

SA-CASSCF/cc-pVTZ S $\mathrm{S}_{0}$ energy: -518.86308218

SA-CASSCF/cc-pVTZ S $S_{1}$ energy: -518.81347691

$\begin{array}{lrrr}\text { C } & -1.044326 & -0.264372 & 0.082075 \\ \text { C } & 0.330004 & 0.061322 & -0.259300 \\ \text { C } & 1.067649 & 1.242741 & 0.361499 \\ \text { C } & 1.305591 & -0.945164 & -0.564811 \\ \text { C } & 2.579017 & -0.525785 & -0.342839 \\ \text { C } & 2.546155 & 0.948858 & -0.013335 \\ \text { N } & -2.067039 & 0.216150 & -0.599444 \\ \text { C } & -3.323117 & -0.397075 & -0.207591 \\ \text { H } & 2.824432 & 1.531471 & -0.891712 \\ \text { C } & -1.563343 & -1.215153 & 1.048586 \\ \text { C } & -2.879120 & -1.431867 & 0.776621 \\ \text { H } & -3.833151 & -0.802855 & -1.073303 \\ \text { H } & -3.969871 & 0.353197 & 0.239905 \\ \text { H } & 0.758971 & 2.166375 & -0.126371 \\ \text { C } & -2.044722 & 1.397931 & -1.436208 \\ \text { H } & -1.025939 & 1.607864 & -1.703418 \\ \text { H } & -2.475189 & 2.229764 & -0.888117 \\ \text { H } & -2.629206 & 1.230383 & -2.330303 \\ \text { H } & 1.052494 & -1.942278 & -0.881742 \\ \text { H } & 3.238605 & 1.233442 & 0.775549 \\ \text { H } & -0.930204 & -1.774274 & 1.702363 \\ \text { H } & -3.516526 & -2.204728 & 1.150555 \\ \text { C } & 0.860759 & 1.474331 & 1.858474 \\ \text { H } & -0.187452 & 1.632741 & 2.099055 \\ \text { H } & 1.402133 & 2.356936 & 2.184208 \\ \text { H } & 1.211809 & 0.630221 & 2.443171 \\ \text { C } & 3.856948 & -1.272106 & -0.558019 \\ \text { H } & 3.674139 & -2.324765 & -0.743413 \\ \text { H } & 4.422531 & -0.883988 & -1.404630 \\ \text { H } & 4.509415 & -1.199583 & 0.309663\end{array}$


2-E, MEP point $10\left(\mathrm{~S}_{1}\right)$

SA-CASSCF/cc-pVTZ S $\mathrm{S}_{0}$ energy: -518.85132344

SA-CASSCF/cc-pVTZ S S $_{1}$ energy: -518.82135944

$\begin{array}{lrrc}\text { C } & -1.035774 & -0.232162 & 0.067501 \\ \text { C } & 0.323814 & 0.109580 & -0.274098 \\ \text { C } & 1.082773 & 1.236644 & 0.423950 \\ \text { C } & 1.295693 & -0.900019 & -0.633306 \\ \text { C } & 2.568294 & -0.515854 & -0.375918 \\ \text { C } & 2.558339 & 0.936977 & 0.040564 \\ \text { N } & -2.067623 & 0.173764 & -0.647453 \\ \text { C } & -3.315480 & -0.434885 & -0.227923 \\ \text { H } & 2.854302 & 1.565810 & -0.798923 \\ \text { C } & -1.563454 & -1.135284 & 1.101636 \\ \text { C } & -2.867464 & -1.371459 & 0.848811 \\ \text { H } & -3.796592 & -0.930647 & -1.062834 \\ \text { H } & -3.990887 & 0.331127 & 0.140123 \\ \text { H } & 0.796385 & 2.190762 & -0.016247 \\ \text { C } & -2.066700 & 1.331380 & -1.520207 \\ \text { H } & -1.045825 & 1.599936 & -1.717699 \\ \text { H } & -2.588908 & 2.142592 & -1.024787 \\ \text { H } & -2.572665 & 1.105113 & -2.448000 \\ \text { H } & 1.031071 & -1.870004 & -1.018483 \\ \text { H } & 3.250245 & 1.160191 & 0.849839 \\ \text { H } & -0.929544 & -1.640544 & 1.797421 \\ \text { H } & -3.504409 & -2.109344 & 1.289662 \\ \text { C } & 0.874163 & 1.404617 & 1.929548 \\ \text { H } & -0.172357 & 1.569998 & 2.173722 \\ \text { H } & 1.427588 & 2.263968 & 2.295132 \\ \text { H } & 1.209950 & 0.531813 & 2.480445 \\ \text { C } & 3.839015 & -1.276139 & -0.591430 \\ \text { H } & 3.643971 & -2.317188 & -0.823776 \\ \text { H } & 4.433573 & -0.864384 & -1.406132 \\ \text { H } & 4.468658 & -1.249751 & 0.295455\end{array}$


2-E, MEP point $11\left(\mathrm{~S}_{1}\right)$

SA-CASSCF/cc-pVTZ S $\mathrm{S}_{0}$ energy: -518.84316541

SA-CASSCF/cc-pVTZ S 1 energy: -518.82845758

$\begin{array}{lrrc}\text { C } & -1.027566 & -0.196992 & 0.050719 \\ \text { C } & 0.318160 & 0.168037 & -0.295551 \\ \text { C } & 1.095676 & 1.236044 & 0.479759 \\ \text { C } & 1.282117 & -0.853326 & -0.675833 \\ \text { C } & 2.556013 & -0.504610 & -0.394494 \\ \text { C } & 2.569069 & 0.925282 & 0.095612 \\ \text { N } & -2.069728 & 0.153426 & -0.671552 \\ \text { C } & -3.305391 & -0.464763 & -0.233418 \\ \text { H } & 2.888368 & 1.590586 & -0.706139 \\ \text { C } & -1.550369 & -1.067260 & 1.133881 \\ \text { C } & -2.851707 & -1.310840 & 0.913455 \\ \text { H } & -3.746018 & -1.036407 & -1.041141 \\ \text { H } & -4.015090 & 0.299327 & 0.063822 \\ \text { H } & 0.839699 & 2.223259 & 0.099046 \\ \text { C } & -2.088064 & 1.247377 & -1.623060 \\ \text { H } & -1.071331 & 1.560355 & -1.777164 \\ \text { H } & -2.686333 & 2.051166 & -1.210320 \\ \text { H } & -2.523897 & 0.929082 & -2.559083 \\ \text { H } & 1.005271 & -1.802085 & -1.104274 \\ \text { H } & 3.255532 & 1.091451 & 0.923291 \\ \text { H } & -0.913449 & -1.514343 & 1.865624 \\ \text { H } & -3.490845 & -2.002993 & 1.420554 \\ \text { C } & 0.883341 & 1.317727 & 1.993852 \\ \text { H } & -0.159392 & 1.496917 & 2.245066 \\ \text { H } & 1.456394 & 2.140171 & 2.410865 \\ \text { H } & 1.195855 & 0.406452 & 2.494603 \\ \text { C } & 3.815570 & -1.278008 & -0.631844 \\ \text { H } & 3.604155 & -2.299905 & -0.926347 \\ \text { H } & 4.433820 & -0.833304 & -1.410494 \\ \text { H } & 4.427831 & -1.314884 & 0.266709\end{array}$


2-E, MEP point $12\left(\mathrm{~S}_{1}\right)$

SA-CASSCF/cc-pVTZ S $\mathrm{S}_{0}$ energy: -518.83722842

SA-CASSCF/cc-pVTZ S S $_{1}$ energy: -518.83384297

$\begin{array}{lrrc}\text { C } & -1.020489 & -0.156361 & 0.031770 \\ \text { C } & 0.314706 & 0.235786 & -0.324343 \\ \text { C } & 1.106436 & 1.240642 & 0.527631 \\ \text { C } & 1.266201 & -0.811118 & -0.690315 \\ \text { C } & 2.541633 & -0.497395 & -0.385514 \\ \text { C } & 2.576148 & 0.918357 & 0.143322 \\ \text { N } & -2.074922 & 0.149715 & -0.685240 \\ \text { C } & -3.296429 & -0.477008 & -0.220549 \\ \text { H } & 2.910850 & 1.598351 & -0.640078 \\ \text { C } & -1.524130 & -1.013173 & 1.147327 \\ \text { C } & -2.827577 & -1.256799 & 0.966133 \\ \text { H } & -3.714826 & -1.099405 & -1.002900 \\ \text { H } & -4.028002 & 0.280606 & 0.034925 \\ \text { H } & 0.878097 & 2.257581 & 0.217440 \\ \text { C } & -2.110293 & 1.150669 & -1.734352 \\ \text { H } & -1.103038 & 1.499132 & -1.876019 \\ \text { H } & -2.764934 & 1.954798 & -1.420421 \\ \text { H } & -2.490859 & 0.722441 & -2.651304 \\ \text { H } & 0.979091 & -1.737201 & -1.161100 \\ \text { H } & 3.259659 & 1.052091 & 0.979379 \\ \text { H } & -0.876642 & -1.420047 & 1.892382 \\ \text { H } & -3.465299 & -1.905692 & 1.529470 \\ \text { C } & 0.890433 & 1.213664 & 2.045682 \\ \text { H } & -0.146550 & 1.405202 & 2.310430 \\ \text { H } & 1.487433 & 1.983822 & 2.524872 \\ \text { H } & 1.175636 & 0.258438 & 2.476037 \\ \text { C } & 3.788514 & -1.275587 & -0.671691 \\ \text { H } & 3.559318 & -2.274121 & -1.027474 \\ \text { H } & 4.411555 & -0.793448 & -1.423889 \\ \text { H } & 4.402391 & -1.377315 & 0.220817\end{array}$


2-Z, $\mathrm{S}_{0}$ geometry

CASSCF/cc-pVTZ S $\mathrm{S}_{0}$ energy: -518.95982731

$\begin{array}{lrcc}\text { C } & 1.05736544 & -0.11452398 & -0.21194142 \\ \text { C } & -0.28945738 & -0.11145527 & -0.11095564 \\ \text { C } & -1.14437542 & -1.38076373 & -0.18133528 \\ \text { C } & -1.23197987 & 1.00719437 & 0.01608278 \\ \text { C } & -2.49926342 & 0.61676187 & -0.18347416 \\ \text { C } & -2.56030538 & -0.85474031 & -0.50861837 \\ \text { N } & 1.96520455 & 0.96227261 & -0.17713207 \\ \text { C } & 3.32804817 & 0.46427287 & -0.10489621 \\ \text { H } & -2.80781192 & -0.99541967 & -1.55801060 \\ \text { C } & 1.87961456 & -1.31309438 & -0.43434766 \\ \text { C } & 3.17580028 & -0.99639394 & -0.39685970 \\ \text { H } & 3.76591044 & 0.61946389 & 0.88304641 \\ \text { H } & 3.96875452 & 0.97198776 & -0.82049420 \\ \text { H } & -0.80271067 & -2.04084345 & -0.96810440 \\ \text { C } & 1.69315975 & 2.18888314 & 0.52378635 \\ \text { H } & 1.33575360 & 2.02422925 & 1.53968553 \\ \text { H } & 0.96953591 & 2.79546257 & -0.00112421 \\ \text { H } & 2.60631077 & 2.76604652 & 0.57976550 \\ \text { H } & -0.95648246 & 2.01846664 & 0.22558256 \\ \text { H } & -3.32651473 & -1.37047737 & 0.06189001 \\ \text { H } & 1.47672955 & -2.28733863 & -0.60973738 \\ \text { H } & 4.00341478 & -1.66324379 & -0.52772192 \\ \text { C } & -1.13574562 & -2.15254439 & 1.14111100 \\ \text { H } & -1.50050300 & -1.52807641 & 1.94973657 \\ \text { H } & -0.13745331 & -2.48031220 & 1.40349554 \\ \text { H } & -1.77308308 & -3.02902933 & 1.08043208 \\ \text { C } & -3.72357850 & 1.47645911 & -0.16839028 \\ \text { H } & -3.48313994 & 2.50842527 & 0.05404412 \\ \text { H } & -4.22933759 & 1.44776091 & -1.12926278 \\ \text { H } & -4.43487203 & 1.12784605 & 0.57447878\end{array}$


2-Z, MEP point $1\left(\mathrm{~S}_{1}\right)$

SA-CASSCF/cc-pVTZ S $\mathrm{S}_{0}$ energy: -518.91956796

SA-CASSCF/cc-pVTZ S $S_{1}$ energy: -518.79659049

$\begin{array}{lrrc}\mathrm{C} & 1.112629 & -0.151739 & -0.198133 \\ \mathrm{C} & -0.333173 & -0.082735 & -0.105181 \\ \mathrm{C} & -1.158512 & -1.362558 & -0.179854 \\ \mathrm{C} & -1.194780 & 1.001314 & -0.028239 \\ \mathrm{C} & -2.543235 & 0.610157 & -0.159565 \\ \mathrm{C} & -2.583385 & -0.855023 & -0.495201 \\ \mathrm{~N} & 1.957357 & 0.910452 & -0.087927 \\ \mathrm{C} & 3.340274 & 0.469175 & -0.104852 \\ \mathrm{H} & -2.820183 & -0.988152 & -1.550344 \\ \mathrm{C} & 1.850931 & -1.334408 & -0.426629 \\ \mathrm{C} & 3.268012 & -0.969581 & -0.472900 \\ \mathrm{H} & 3.775363 & 0.606751 & 0.884848 \\ \mathrm{H} & 3.921844 & 1.066557 & -0.801117 \\ \mathrm{H} & -0.803521 & -2.006747 & -0.976211 \\ \mathrm{C} & 1.667901 & 2.195853 & 0.498095 \\ \mathrm{H} & 1.219510 & 2.100379 & 1.481278 \\ \mathrm{H} & 1.016307 & 2.786048 & -0.128765 \\ \mathrm{H} & 2.596997 & 2.737340 & 0.600616 \\ \mathrm{H} & -0.912985 & 2.023351 & 0.107569 \\ \mathrm{H} & -3.342206 & -1.395330 & 0.061693 \\ \mathrm{H} & 1.438945 & -2.274724 & -0.708478 \\ \mathrm{H} & 4.090639 & -1.637997 & -0.322049 \\ \mathrm{C} & -1.121991 & -2.148403 & 1.136372 \\ \mathrm{H} & -1.527936 & -1.547431 & 1.943478 \\ \mathrm{H} & -0.113443 & -2.433108 & 1.406426 \\ \mathrm{H} & -1.717915 & -3.051991 & 1.059002 \\ \mathrm{C} & -3.741958 & 1.495023 & -0.170578 \\ \mathrm{H} & -3.482404 & 2.521362 & 0.060384 \\ \mathrm{H} & -4.233058 & 1.488278 & -1.141941 \\ \mathrm{H} & -4.482330 & 1.169595 & 0.555681\end{array}$


2-Z, MEP point $2\left(\mathrm{~S}_{1}\right)$

SA-CASSCF/cc-pVTZ S $\mathrm{S}_{0}$ energy: -518.91533772

SA-CASSCF/cc-pVTZ S $S_{1}$ energy: -518.79831259

$\begin{array}{lrrr}\text { C } & 1.114663 & -0.170812 & -0.186540 \\ \text { C } & -0.333390 & -0.092209 & -0.103134 \\ \text { C } & -1.169113 & -1.364339 & -0.160683 \\ \text { C } & -1.190075 & 1.004819 & -0.081049 \\ \text { C } & -2.535879 & 0.627350 & -0.179521 \\ \text { C } & -2.603943 & -0.853758 & -0.436941 \\ \text { N } & 1.948497 & 0.868230 & 0.036834 \\ \text { C } & 3.336350 & 0.474103 & -0.092426 \\ \text { H } & -2.894138 & -1.039327 & -1.470126 \\ \text { C } & 1.868700 & -1.336422 & -0.479567 \\ \text { C } & 3.279877 & -0.947468 & -0.530494 \\ \text { H } & 3.834730 & 0.583367 & 0.868917 \\ \text { H } & 3.845454 & 1.124136 & -0.799400 \\ \text { H } & -0.837282 & -2.002543 & -0.972538 \\ \text { C } & 1.628894 & 2.199171 & 0.481864 \\ \text { H } & 0.965796 & 2.179523 & 1.335481 \\ \text { H } & 1.175489 & 2.792822 & -0.302635 \\ \text { H } & 2.546188 & 2.686628 & 0.778576 \\ \text { H } & -0.895317 & 2.030055 & -0.006472 \\ \text { H } & -3.340516 & -1.355347 & 0.182982 \\ \text { H } & 1.459781 & -2.240432 & -0.868854 \\ \text { H } & 4.108208 & -1.609030 & -0.369080 \\ \text { C } & -1.091419 & -2.163004 & 1.144618 \\ \text { H } & -1.489639 & -1.577649 & 1.967040 \\ \text { H } & -0.071764 & -2.432726 & 1.387102 \\ \text { H } & -1.672991 & -3.076303 & 1.070972 \\ \text { C } & -3.728305 & 1.524275 & -0.199213 \\ \text { H } & -3.455721 & 2.553812 & 0.000222 \\ \text { H } & -4.232808 & 1.495885 & -1.163100 \\ \text { H } & -4.461227 & 1.225630 & 0.545713\end{array}$


2-Z, MEP point $3\left(\mathrm{~S}_{1}\right)$

SA-CASSCF/cc-pVTZ S $\mathrm{S}_{0}$ energy: -518.91344892

SA-CASSCF/cc-pVTZ S $S_{1}$ energy: -518.79924133

$\begin{array}{cccc}\text { C } & 1.109134 & -0.168606 & -0.201774 \\ \text { C } & -0.335904 & -0.095809 & -0.077969 \\ \text { C } & -1.174866 & -1.366322 & -0.116116 \\ \text { C } & -1.185691 & 1.008550 & -0.147856 \\ \text { C } & -2.528583 & 0.641130 & -0.233193 \\ \text { C } & -2.621569 & -0.856860 & -0.346356 \\ \text { N } & 1.941953 & 0.848813 & 0.100633 \\ \text { C } & 3.329940 & 0.473415 & -0.078712 \\ \text { H } & -2.989856 & -1.141927 & -1.329889 \\ \text { C } & 1.862856 & -1.298395 & -0.606623 \\ \text { C } & 3.277181 & -0.940874 & -0.543644 \\ \text { H } & 3.859747 & 0.580677 & 0.864212 \\ \text { H } & 3.803977 & 1.138699 & -0.797136 \\ \text { H } & -0.869983 & -1.989415 & -0.951128 \\ \text { C } & 1.616543 & 2.182451 & 0.531299 \\ \text { H } & 0.843328 & 2.167586 & 1.283797 \\ \text { H } & 1.295755 & 2.810845 & -0.292767 \\ \text { H } & 2.501513 & 2.626038 & 0.965174 \\ \text { H } & -0.878876 & 2.033578 & -0.148006 \\ \text { H } & -3.316680 & -1.281307 & 0.372465 \\ \text { H } & 1.456121 & -2.216220 & -0.961030 \\ \text { H } & 4.083209 & -1.619623 & -0.343312 \\ \text { C } & -1.051176 & -2.192250 & 1.166801 \\ \text { H } & -1.407450 & -1.621626 & 2.018010 \\ \text { H } & -0.024572 & -2.476775 & 1.359641 \\ \text { H } & -1.643739 & -3.099022 & 1.099601 \\ \text { C } & -3.715589 & 1.545005 & -0.257152 \\ \text { H } & -3.426483 & 2.584413 & -0.156570 \\ \text { H } & -4.274958 & 1.444575 & -1.184804 \\ \text { H } & -4.406325 & 1.313834 & 0.550367\end{array}$


2-Z, MEP point $4\left(\mathrm{~S}_{1}\right)$

SA-CASSCF/cc-pVTZ S $\mathrm{S}_{0}$ energy: -518.89513412

SA-CASSCF/cc-pVTZ S $S_{1}$ energy: -518.79411325

$\begin{array}{lrrc}\text { C } & 1.101978 & -0.154511 & -0.226837 \\ \text { C } & -0.337332 & -0.091357 & -0.049871 \\ \text { C } & -1.177142 & -1.362041 & -0.067951 \\ \text { C } & -1.185511 & 1.010522 & -0.217427 \\ \text { C } & -2.522530 & 0.648008 & -0.287153 \\ \text { C } & -2.633724 & -0.852630 & -0.237734 \\ \text { N } & 1.937152 & 0.837112 & 0.137191 \\ \text { C } & 3.323819 & 0.463761 & -0.062608 \\ \text { H } & -3.089303 & -1.240548 & -1.145451 \\ \text { C } & 1.851373 & -1.243088 & -0.750813 \\ \text { C } & 3.261301 & -0.947734 & -0.535724 \\ \text { H } & 3.867834 & 0.573900 & 0.870053 \\ \text { H } & 3.783731 & 1.127903 & -0.792292 \\ \text { H } & -0.907416 & -1.961341 & -0.933245 \\ \text { C } & 1.612150 & 2.160184 & 0.600004 \\ \text { H } & 0.785175 & 2.130400 & 1.291845 \\ \text { H } & 1.365017 & 2.829241 & -0.217717 \\ \text { H } & 2.472107 & 2.563978 & 1.115379 \\ \text { H } & -0.866410 & 2.030465 & -0.292566 \\ \text { H } & -3.268373 & -1.179638 & 0.582272 \\ \text { H } & 1.443624 & -2.193430 & -1.005071 \\ \text { H } & 4.024375 & -1.656303 & -0.278676 \\ \text { C } & -1.002035 & -2.225349 & 1.182133 \\ \text { H } & -1.310013 & -1.677284 & 2.066208 \\ \text { H } & 0.029602 & -2.526109 & 1.317744 \\ \text { H } & -1.606549 & -3.124576 & 1.118016 \\ \text { C } & -3.707666 & 1.554842 & -0.328016 \\ \text { H } & -3.410931 & 2.596695 & -0.348459 \\ \text { H } & -4.322425 & 1.367064 & -1.204886 \\ \text { H } & -4.347256 & 1.409138 & 0.539352\end{array}$


2-Z, MEP point $5\left(\mathrm{~S}_{1}\right)$

SA-CASSCF/cc-pVTZ S $\mathrm{S}_{0}$ energy: -518.89957076

SA-CASSCF/cc-pVTZ S $S_{1}$ energy: -518.80216958

$\begin{array}{cccc}\text { C } & 1.095091 & -0.141108 & -0.243979 \\ \text { C } & -0.339045 & -0.084467 & -0.030766 \\ \text { C } & -1.181708 & -1.353886 & -0.022183 \\ \text { C } & -1.184497 & 1.007877 & -0.291800 \\ \text { C } & -2.518177 & 0.649548 & -0.336837 \\ \text { C } & -2.640922 & -0.834876 & -0.111522 \\ \text { N } & 1.932411 & 0.827958 & 0.164813 \\ \text { C } & 3.316916 & 0.452072 & -0.045810 \\ \text { H } & -3.194076 & -1.319881 & -0.911133 \\ \text { C } & 1.838091 & -1.198786 & -0.838713 \\ \text { C } & 3.239360 & -0.945560 & -0.556745 \\ \text { H } & 3.863005 & 0.537610 & 0.887596 \\ \text { H } & 3.779405 & 1.128496 & -0.762799 \\ \text { H } & -0.963322 & -1.927276 & -0.920815 \\ \text { C } & 1.606607 & 2.132685 & 0.677863 \\ \text { H } & 0.726832 & 2.085829 & 1.298484 \\ \text { H } & 1.439271 & 2.850710 & -0.119189 \\ \text { H } & 2.433058 & 2.485084 & 1.279643 \\ \text { H } & -0.853401 & 2.016244 & -0.445757 \\ \text { H } & -3.188898 & -1.043014 & 0.805321 \\ \text { H } & 1.421932 & -2.135923 & -1.129598 \\ \text { H } & 3.989158 & -1.675084 & -0.322430 \\ \text { C } & -0.947905 & -2.259320 & 1.185854 \\ \text { H } & -1.190219 & -1.736460 & 2.104629 \\ \text { H } & 0.083463 & -2.584110 & 1.247745 \\ \text { H } & -1.572808 & -3.145130 & 1.129869 \\ \text { C } & -3.700008 & 1.559306 & -0.413479 \\ \text { H } & -3.400098 & 2.591181 & -0.552090 \\ \text { H } & -4.356807 & 1.289699 & -1.236156 \\ \text { H } & -4.297366 & 1.506365 & 0.494510\end{array}$


2-Z, MEP point $6\left(\mathrm{~S}_{1}\right)$

SA-CASSCF/cc-pVTZ S $\mathrm{S}_{0}$ energy: -518.89529079

SA-CASSCF/cc-pVTZ S S $_{1}$ energy: -518.80354019

$\begin{array}{lrrr}\text { C } & 1.088410 & -0.129375 & -0.252637 \\ \text { C } & -0.341688 & -0.075440 & -0.011871 \\ \text { C } & -1.188850 & -1.341131 & 0.025093 \\ \text { C } & -1.182786 & 0.998073 & -0.367244 \\ \text { C } & -2.516422 & 0.644382 & -0.383069 \\ \text { C } & -2.640840 & -0.803903 & 0.016282 \\ \text { N } & 1.928606 & 0.816511 & 0.192870 \\ \text { C } & 3.310599 & 0.442426 & -0.035983 \\ \text { H } & -3.280369 & -1.369015 & -0.655300 \\ \text { C } & 1.823277 & -1.162332 & -0.895209 \\ \text { C } & 3.220822 & -0.930683 & -0.609943 \\ \text { H } & 3.857071 & 0.479910 & 0.900266 \\ \text { H } & 3.778134 & 1.147935 & -0.720612 \\ \text { H } & -1.029629 & -1.890216 & -0.902838 \\ \text { C } & 1.603184 & 2.096466 & 0.766243 \\ \text { H } & 0.679559 & 2.034754 & 1.316670 \\ \text { H } & 1.511358 & 2.864694 & 0.004520 \\ \text { H } & 2.392941 & 2.387853 & 1.445553 \\ \text { H } & -0.840408 & 1.989651 & -0.595757 \\ \text { H } & -3.089763 & -0.883528 & 1.005893 \\ \text { H } & 1.396467 & -2.073101 & -1.248049 \\ \text { H } & 3.975169 & -1.670442 & -0.428948 \\ \text { C } & -0.902107 & -2.286744 & 1.188349 \\ \text { H } & -1.075939 & -1.789350 & 2.136303 \\ \text { H } & 0.123749 & -2.634539 & 1.176170 \\ \text { H } & -1.547630 & -3.158418 & 1.143990 \\ \text { C } & -3.693815 & 1.554615 & -0.509982 \\ \text { H } & -3.391238 & 2.562732 & -0.767202 \\ \text { H } & -4.383420 & 1.208419 & -1.274717 \\ \text { H } & -4.256228 & 1.603748 & 0.420440\end{array}$


2-Z, MEP point $7\left(\mathrm{~S}_{1}\right)$

SA-CASSCF/cc-pVTZ S $\mathrm{S}_{0}$ energy: -518.89089774

SA-CASSCF/cc-pVTZ S S $_{1}$ energy: -518.80465940

\begin{tabular}{|c|c|c|c|}
\hline $\mathrm{C}$ & 1.082854 & -0.116139 & -0.256438 \\
\hline $\mathrm{C}$ & -0.343897 & -0.064527 & 0.009242 \\
\hline $\mathrm{C}$ & -1.192764 & -1.327876 & 0.075981 \\
\hline $\mathrm{C}$ & -1.182451 & 0.982788 & -0.430497 \\
\hline $\mathrm{C}$ & -2.515737 & 0.630502 & -0.426953 \\
\hline $\mathrm{C}$ & -2.635589 & -0.774347 & 0.110554 \\
\hline $\mathbf{N}$ & 1.929456 & 0.797990 & 0.229607 \\
\hline $\mathrm{C}$ & 3.307167 & 0.430170 & -0.032076 \\
\hline $\mathrm{H}$ & -3.324645 & -1.391849 & -0.456916 \\
\hline $\mathrm{C}$ & 1.805759 & -1.118227 & -0.955592 \\
\hline $\mathrm{C}$ & 3.198940 & -0.908292 & -0.680377 \\
\hline $\mathrm{H}$ & 3.862255 & 0.411057 & 0.899660 \\
\hline $\mathrm{H}$ & 3.772771 & 1.170695 & -0.679746 \\
\hline $\mathrm{H}$ & -1.076028 & -1.869782 & -0.864374 \\
\hline $\mathrm{C}$ & 1.610766 & 2.054461 & 0.858268 \\
\hline $\mathrm{H}$ & 0.641376 & 1.998388 & 1.321880 \\
\hline $\mathrm{H}$ & 1.615860 & 2.866659 & 0.138058 \\
\hline $\mathrm{H}$ & 2.350606 & 2.270223 & 1.617904 \\
\hline $\mathrm{H}$ & -0.832793 & 1.955208 & -0.725124 \\
\hline $\mathrm{H}$ & -3.012763 & -0.748523 & 1.133840 \\
\hline $\mathrm{H}$ & 1.366085 & -1.994438 & -1.374296 \\
\hline $\mathrm{H}$ & 3.966658 & -1.650127 & -0.593101 \\
\hline $\mathrm{C}$ & -0.874365 & -2.290430 & 1.214569 \\
\hline $\mathrm{H}$ & -1.001972 & -1.805127 & 2.175584 \\
\hline $\mathrm{H}$ & 0.145842 & -2.651572 & 1.154902 \\
\hline $\mathrm{H}$ & -1.530998 & -3.154382 & 1.184220 \\
\hline $\mathrm{C}$ & -3.690621 & 1.536922 & -0.602724 \\
\hline $\mathrm{H}$ & -3.387201 & 2.516103 & -0.953894 \\
\hline $\mathrm{H}$ & -4.401869 & 1.132640 & -1.317631 \\
\hline $\mathrm{H}$ & -4.229421 & 1.672098 & 0.333562 \\
\hline
\end{tabular}


2-Z, MEP point $8\left(\mathrm{~S}_{1}\right)$

SA-CASSCF/cc-pVTZ S $\mathrm{S}_{0}$ energy: -518.88555047

SA-CASSCF/cc-pVTZ S $S_{1}$ energy: -518.80615993

$\begin{array}{crrr}\text { C } & 1.079576 & -0.096450 & -0.261328 \\ \text { C } & -0.347146 & -0.045632 & 0.028915 \\ \text { C } & -1.191661 & -1.311485 & 0.130118 \\ \text { C } & -1.183499 & 0.956721 & -0.507111 \\ \text { C } & -2.518565 & 0.604837 & -0.483349 \\ \text { C } & -2.627661 & -0.749315 & 0.174337 \\ \text { N } & 1.934650 & 0.774773 & 0.268606 \\ \text { C } & 3.307527 & 0.412723 & -0.028474 \\ \text { H } & -3.341426 & -1.409984 & -0.306847 \\ \text { C } & 1.790528 & -1.064070 & -1.017411 \\ \text { C } & 3.168808 & -0.884437 & -0.750494 \\ \text { H } & 3.874848 & 0.336440 & 0.892933 \\ \text { H } & 3.769858 & 1.186450 & -0.638590 \\ \text { H } & -1.097506 & -1.862307 & -0.809990 \\ \text { C } & 1.628361 & 2.004935 & 0.956486 \\ \text { H } & 0.616940 & 1.973357 & 1.319269 \\ \text { H } & 1.748446 & 2.853528 & 0.290669 \\ \text { H } & 2.303202 & 2.129310 & 1.793396 \\ \text { H } & -0.832604 & 1.911049 & -0.857256 \\ \text { H } & -2.957662 & -0.630185 & 1.208252 \\ \text { H } & 1.333446 & -1.897304 & -1.501175 \\ \text { H } & 3.960749 & -1.597742 & -0.835862 \\ \text { C } & -0.860113 & -2.273838 & 1.263068 \\ \text { H } & -0.969549 & -1.789036 & 2.226455 \\ \text { H } & 0.157791 & -2.640352 & 1.186400 \\ \text { H } & -1.520236 & -3.135671 & 1.242086 \\ \text { C } & -3.690924 & 1.512131 & -0.671542 \\ \text { H } & -3.392708 & 2.461126 & -1.101607 \\ \text { H } & -4.437078 & 1.071031 & -1.326305 \\ \text { H } & -4.188316 & 1.719670 & 0.275036\end{array}$


2-Z, MEP point $9\left(\mathrm{~S}_{1}\right)$

SA-CASSCF/cc-pVTZ S $\mathrm{S}_{0}$ energy: -518.87404533

SA-CASSCF/cc-pVTZ S $S_{1}$ energy: -518.80995691

$\begin{array}{cccc}\text { C } & 1.074894 & -0.072986 & -0.262694 \\ \text { C } & -0.355256 & -0.016620 & 0.043586 \\ \text { C } & -1.187629 & -1.288351 & 0.190849 \\ \text { C } & -1.190180 & 0.917538 & -0.600060 \\ \text { C } & -2.527387 & 0.568479 & -0.548849 \\ \text { C } & -2.623107 & -0.730630 & 0.217027 \\ \text { N } & 1.943318 & 0.735498 & 0.325068 \\ \text { C } & 3.310758 & 0.384951 & -0.011072 \\ \text { H } & -3.340094 & -1.432060 & -0.198396 \\ \text { C } & 1.789278 & -1.000608 & -1.083596 \\ \text { C } & 3.137175 & -0.851926 & -0.830491 \\ \text { H } & 3.891104 & 0.231000 & 0.892116 \\ \text { H } & 3.772976 & 1.198329 & -0.566732 \\ \text { H } & -1.100737 & -1.853472 & -0.745188 \\ \text { C } & 1.651158 & 1.958910 & 1.037064 \\ \text { H } & 0.609338 & 1.974341 & 1.297910 \\ \text { H } & 1.892937 & 2.812017 & 0.410959 \\ \text { H } & 2.249271 & 2.012280 & 1.937016 \\ \text { H } & -0.842311 & 1.851897 & -1.007591 \\ \text { H } & -2.933895 & -0.529499 & 1.246292 \\ \text { H } & 1.317459 & -1.787354 & -1.629672 \\ \text { H } & 3.935258 & -1.520945 & -1.070167 \\ \text { C } & -0.850821 & -2.247913 & 1.321693 \\ \text { H } & -0.955431 & -1.766507 & 2.287321 \\ \text { H } & 0.168239 & -2.612968 & 1.239299 \\ \text { H } & -1.506284 & -3.113485 & 1.300930 \\ \text { C } & -3.693101 & 1.491822 & -0.712764 \\ \text { H } & -3.399335 & 2.418097 & -1.193819 \\ \text { H } & -4.480059 & 1.043356 & -1.313330 \\ \text { H } & -4.139110 & 1.745838 & 0.248915\end{array}$


2-Z, MEP point $10\left(\mathrm{~S}_{1}\right)$

SA-CASSCF/cc-pVTZ S $\mathrm{S}_{0}$ energy: -518.86092218

SA-CASSCF/cc-pVTZ S S $_{1}$ energy: -518.81628911

$\begin{array}{rrrr}\text { C } & 1.066568 & -0.051468 & -0.266007 \\ \text { C } & -0.358748 & 0.007751 & 0.033207 \\ \text { C } & -1.184884 & -1.264778 & 0.246045 \\ \text { C } & -1.207299 & 0.876648 & -0.695421 \\ \text { C } & -2.536893 & 0.534058 & -0.595513 \\ \text { C } & -2.619196 & -0.710756 & 0.258529 \\ \text { N } & 1.953320 & 0.688643 & 0.384145 \\ \text { C } & 3.314478 & 0.348806 & 0.015577 \\ \text { H } & -3.339408 & -1.440973 & -0.096704 \\ \text { C } & 1.785442 & -0.927075 & -1.163396 \\ \text { C } & 3.117514 & -0.811613 & -0.907348 \\ \text { H } & 3.900240 & 0.108579 & 0.895839 \\ \text { H } & 3.785546 & 1.198064 & -0.473348 \\ \text { H } & -1.104391 & -1.855748 & -0.675523 \\ \text { C } & 1.676280 & 1.905308 & 1.116596 \\ \text { H } & 0.619373 & 1.965161 & 1.298686 \\ \text { H } & 2.008557 & 2.757754 & 0.531641 \\ \text { H } & 2.207468 & 1.905347 & 2.059327 \\ \text { H } & -0.869806 & 1.786738 & -1.160853 \\ \text { H } & -2.912093 & -0.439569 & 1.276657 \\ \text { H } & 1.303232 & -1.662531 & -1.769830 \\ \text { H } & 3.912090 & -1.444637 & -1.242012 \\ \text { C } & -0.839404 & -2.205536 & 1.388144 \\ \text { H } & -0.942012 & -1.716141 & 2.350175 \\ \text { H } & 0.180684 & -2.569204 & 1.305349 \\ \text { H } & -1.492258 & -3.073142 & 1.377148 \\ \text { C } & -3.697608 & 1.466623 & -0.753377 \\ \text { H } & -3.412457 & 2.366585 & -1.286383 \\ \text { H } & -4.519595 & 1.007526 & -1.295656 \\ \text { H } & -4.092245 & 1.769606 & 0.216604\end{array}$


2-Z, MEP point $11\left(\mathrm{~S}_{1}\right)$

SA-CASSCF/cc-pVTZ S $\mathrm{S}_{0}$ energy: -518.84933515

SA-CASSCF/cc-pVTZ S $S_{1}$ energy: -518.82334835

$\begin{array}{lrrr}\text { C } & 1.057789 & -0.031967 & -0.273962 \\ \text { C } & -0.357985 & 0.027859 & 0.015065 \\ \text { C } & -1.178334 & -1.240113 & 0.292373 \\ \text { C } & -1.226771 & 0.842931 & -0.773389 \\ \text { C } & -2.544519 & 0.507437 & -0.625647 \\ \text { C } & -2.612974 & -0.687616 & 0.299233 \\ \text { N } & 1.958409 & 0.640020 & 0.427038 \\ \text { C } & 3.315413 & 0.307348 & 0.038466 \\ \text { H } & -3.336861 & -1.437699 & -0.004898 \\ \text { C } & 1.781114 & -0.852496 & -1.241676 \\ \text { C } & 3.104064 & -0.765933 & -0.982339 \\ \text { H } & 3.890296 & -0.020874 & 0.897197 \\ \text { H } & 3.806028 & 1.184833 & -0.373823 \\ \text { H } & -1.111168 & -1.866167 & -0.607843 \\ \text { C } & 1.697429 & 1.834197 & 1.205988 \\ \text { H } & 0.634788 & 1.929869 & 1.331866 \\ \text { H } & 2.098844 & 2.692328 & 0.676114 \\ \text { H } & 2.175261 & 1.768574 & 2.173568 \\ \text { H } & -0.901422 & 1.729948 & -1.290914 \\ \text { H } & -2.889515 & -0.360973 & 1.305546 \\ \text { H } & 1.290686 & -1.529810 & -1.906735 \\ \text { H } & 3.895014 & -1.367221 & -1.377890 \\ \text { C } & -0.821977 & -2.145106 & 1.459757 \\ \text { H } & -0.917519 & -1.630075 & 2.409078 \\ \text { H } & 0.197793 & -2.510940 & 1.379436 \\ \text { H } & -1.473216 & -3.014243 & 1.477864 \\ \text { C } & -3.708770 & 1.432841 & -0.806882 \\ \text { H } & -3.435484 & 2.301099 & -1.396072 \\ \text { H } & -4.547596 & 0.948821 & -1.299332 \\ \text { H } & -4.073541 & 1.790291 & 0.156051\end{array}$


2-Z, MEP point $12\left(\mathrm{~S}_{1}\right)$

SA-CASSCF/cc-pVTZ S $\mathrm{S}_{0}$ energy: -518.84101031

SA-CASSCF/cc-pVTZ S 1 energy: -518.83008766

$\begin{array}{rrrr}\text { C } & 1.048678 & -0.016523 & -0.279840 \\ \text { C } & -0.358235 & 0.053352 & 0.008387 \\ \text { C } & -1.168165 & -1.212792 & 0.329812 \\ \text { C } & -1.243416 & 0.822277 & -0.826731 \\ \text { C } & -2.550426 & 0.485071 & -0.651483 \\ \text { C } & -2.603310 & -0.662798 & 0.333069 \\ \text { N } & 1.961727 & 0.601093 & 0.445901 \\ \text { C } & 3.314288 & 0.267704 & 0.046350 \\ \text { H } & -3.332321 & -1.427203 & 0.081494 \\ \text { C } & 1.773549 & -0.791883 & -1.303760 \\ \text { C } & 3.091603 & -0.718972 & -1.055338 \\ \text { H } & 3.866401 & -0.142921 & 0.883162 \\ \text { H } & 3.831388 & 1.159839 & -0.290074 \\ \text { H } & -1.110631 & -1.874387 & -0.546298 \\ \text { C } & 1.716580 & 1.741750 & 1.308272 \\ \text { H } & 0.652796 & 1.861628 & 1.401180 \\ \text { H } & 2.166477 & 2.618061 & 0.854527 \\ \text { H } & 2.158038 & 1.586303 & 2.282513 \\ \text { H } & -0.931461 & 1.691594 & -1.381102 \\ \text { H } & -2.859709 & -0.284390 & 1.326779 \\ \text { H } & 1.274077 & -1.412140 & -2.016164 \\ \text { H } & 3.882750 & -1.271621 & -1.517771 \\ \text { C } & -0.800071 & -2.070488 & 1.529515 \\ \text { H } & -0.887642 & -1.517557 & 2.457985 \\ \text { H } & 0.219621 & -2.439311 & 1.455650 \\ \text { H } & -1.449353 & -2.939250 & 1.588756 \\ \text { C } & -3.725754 & 1.387294 & -0.874898 \\ \text { H } & -3.467286 & 2.220823 & -1.518418 \\ \text { H } & -4.567570 & 0.868939 & -1.325369 \\ \text { H } & -4.080264 & 1.799966 & 0.069788\end{array}$


2-Z, MEP point $13\left(\mathrm{~S}_{1}\right)$

SA-CASSCF/cc-pVTZ S $\mathrm{S}_{0}$ energy: -518.83700760

SA-CASSCF/cc-pVTZ S $S_{1}$ energy: -518.83544199

$\begin{array}{lrrr}\text { C } & 1.040662 & -0.001693 & -0.280680 \\ \mathrm{C} & -0.359754 & 0.084757 & 0.014912 \\ \mathrm{C} & -1.155468 & -1.183146 & 0.359347 \\ \mathrm{C} & -1.256431 & 0.823309 & -0.848263 \\ \mathrm{C} & -2.553856 & 0.467344 & -0.674719 \\ \mathrm{C} & -2.594722 & -0.642794 & 0.353444 \\ \mathrm{~N} & 1.967784 & 0.579771 & 0.445848 \\ \mathrm{C} & 3.314310 & 0.240557 & 0.031973 \\ \mathrm{H} & -3.323389 & -1.419336 & 0.139735 \\ \mathrm{C} & 1.756606 & -0.754963 & -1.343183 \\ \mathrm{C} & 3.075595 & -0.678793 & -1.123481 \\ \mathrm{H} & 3.846827 & -0.232494 & 0.848813 \\ \mathrm{H} & 3.855647 & 1.137161 & -0.247691 \\ \mathrm{H} & -1.096461 & -1.876732 & -0.492931 \\ \mathrm{C} & 1.741356 & 1.632869 & 1.418440 \\ \mathrm{H} & 0.678846 & 1.773382 & 1.501370 \\ \mathrm{H} & 2.228674 & 2.534314 & 1.066115 \\ \mathrm{H} & 2.157877 & 1.358584 & 2.377610 \\ \mathrm{H} & -0.954601 & 1.679096 & -1.429877 \\ \mathrm{H} & -2.841104 & -0.226811 & 1.334737 \\ \mathrm{H} & 1.243623 & -1.330494 & -2.082699 \\ \mathrm{H} & 3.863286 & -1.182941 & -1.643171 \\ \mathrm{C} & -0.777794 & -1.990129 & 1.592653 \\ \mathrm{H} & -0.865870 & -1.398090 & 2.496882 \\ \mathrm{H} & 0.244335 & -2.355777 & 1.531585 \\ \mathrm{H} & -1.420936 & -2.860178 & 1.691942 \\ \mathrm{C} & -3.747119 & 1.329358 & -0.955651 \\ \mathrm{H} & -3.502119 & 2.129331 & -1.645778 \\ \mathrm{H} & -4.575512 & 0.768086 & -1.379420 \\ \mathrm{H} & -4.115858 & 1.789269 & -0.038828\end{array}$

